\title{
Selected Abstracts from Pharmacology 2019
}

\author{
Posters, Monday 16th December, 13:30 \\ Poster session: Clinical
}

\section{P137 | Predictors of serious adverse events in <65-year-aged hypertensive women: A sub-analysis of the SPRINT}

\author{
Volkan Aydin ${ }^{1}$; Ahmet Akici ${ }^{1}$; Sibel Sakarya ${ }^{2}$; Mehmet Akman ${ }^{1}$; \\ Ali Serdar Fak ${ }^{1}$ \\ ${ }^{1}$ Marmara University Hypertension and Atherosclerosis Research Center \\ (HIPAM), Istanbul; ' ${ }^{2}$ Department of Public Health, Koç University School \\ of Medicine, Istanbul
}

Introduction: Women are more likely to experience antihypertensive treatment-related adverse effects in the management of hypertension. ${ }^{1}$ Besides, intensive treatment approaches could increase the rates of serious adverse events (SAEs) during antihypertensive therapy. ${ }^{2}$ We aimed to identify potential basal predictors of SAEs in middle-aged hypertensive women managed with standard or intensive treatment.

Method: Hypertensive patients in the SPRINT, a multicentre controlled trial, were randomized to receive either standard or intensive treatment approach to achieve a target systolic blood pressure of $<140 \mathrm{mmHg}$ or $<120 \mathrm{mmHg}$, respectively. In this sub-analysis, we examined baseline characteristics of the $<65$-year-old hypertensive women in the standard- ( $\mathrm{n}=595)$ or intensive-treatment groups ( $n=652$ ) to identify predictive factors of SAEs by Cox proportionalhazards model.

Results: A total 341 SAEs were identified in the study population, which was observed in $27.6 \%$ and $27.1 \%$ of the standard- and intensive-treatment groups, respectively. Most common SAEs were electrolyte abnormalities (3.5\%) and acute kidney injury/failure (2.8\%). Rates of all SAEs or its individual subclasses were similar in each treatment groups. The risk of overall SAEs was significantly higher in blacks vs. non-blacks (HR 1.27, 95\% Cl: 1.01-1.58), in current smokers vs. non-smokers (HR 1.59, 95\% Cl: 1.23-2.05), and those with vs. without chronic kidney disease (CKD) (HR 1.38, 95\% Cl: 1.08-1.77). These characteristics were found to be associated with also higher risk in the standard-treatment group, whereas this association was not detected in the intensive-treatment group. The likelihood of SAEs significantly increased with age (HR $1.04,95 \% \mathrm{Cl}$ : 1.01-1.07), where the association was preserved in the standardtreatment group and lost in the intensive-treatment group. The rate of hypotension SAE and syncope SAE were increased among CKD patients (HR 6.20, 95\% Cl: 2.02-19.03) and active smokers (HR 4.54, 95\% Cl: 1.17-17.57) at baseline. A reduced rate of electrolyte abnormalities was found in obese patients compared to that in under/normo-weight hypertensive women ( $\mathrm{HR} 0.33,95 \% \mathrm{Cl}$, 0.14-0.77).

Conclusion: Our sub-analysis in middle-aged hypertensive women shows increased risk of SAEs with age, black race, smoking, and CKD, especially in patients where standard treatment strategy was adopted. Consideration of these clinical features by clinicians during choosing a standard or aggressive blood pressure goal may improve rationality of the pharmacotherapy for middle-aged hypertensive women.

\section{REFERENCE(S)}

1. Turnbull F, Woodward M, Neal B, et al. Eur Heart J. 2008;29:26692680.

2. Bress AP, Kramer H, Khatib R, et al. Circulation. 2017;135:1617-1628.

\section{P139 | The pharmacokinetics and relative bioavailability of mepolizumab $100 \mathrm{mg}$ liquid formulation administered subcutaneously to healthy participants: A randomized trial}

Shaila Shabbir ${ }^{1}$; Isabelle Pouliquen ${ }^{1}$; Jane Bentley ${ }^{1}$; Eric Bradford ${ }^{1}$; Morrys Kaisermann ${ }^{1}$; Muna Albayaty ${ }^{2}$

${ }^{1}$ GlaxoSmithKline; ${ }^{2}$ Parexel

Rationale: A new liquid formulation of mepolizumab delivered via a single-use prefilled autoinjector (Al) or prefilled syringe (PFS) is under development to provide convenience and an option to self-administer at home for patients. The aim of this study was to compare the pharmacokinetic (PK) profile of this new liquid formulation with the approved lyophilized formulation of mepolizumab.

Methods: In this open-label, parallel-group, single-dose study, healthy participants were randomized (1:1:1) to receive a single mepolizumab dose (100 mg) administered by a healthcare professional subcutaneously as liquid in a PFS or Al or as reconstituted lyophilized product. Randomization was stratified by body weight $(<70,70-<80$, and $\geq 80 \mathrm{~kg}$ ) and injection site randomized 1:1:1 to upper arm, abdomen, or thigh. Primary endpoints were maximum plasma concentration $\left(C_{\text {max }}\right)$, area under the concentration-time curve (AUC) from time zero (pre-dose) to last time of quantifiable concentration $\left(A \cup C_{[0-t]}\right)$, and AUC from time zero to infinity $\left(\mathrm{AUC}_{[0-\infty]}\right)$. Secondary endpoints included additional PK parameters and safety assessments. Pharmacodynamic effect on blood eosinophil count following administration via 
TABLE 1 Primary study endpoints

\begin{tabular}{|c|c|c|c|c|}
\hline PK parameter & Test treatment & \multicolumn{2}{|c|}{ Adjusted geometric mean (SE [logs]) } & Test/reference ratio $(90 \% \mathrm{Cl})$ \\
\hline $\mathrm{C}_{\max }(\mu \mathrm{g} / \mathrm{mL})$ & PFS & $12.14(0.027)$ & $11.51(0.027)$ & $1.06(0.99,1.12)$ \\
\hline \multirow[t]{2}{*}{$\mathrm{AUC}_{(0-\mathrm{t})}($ day $* \mu \mathrm{g} / \mathrm{mL})$} & PFS & $417.93(0.031)$ & $401.30(0.030)$ & $1.04(0.97,1.12)$ \\
\hline & $\mathrm{Al}$ & $435.46(0.029)$ & $403.00(0.028)$ & $1.08(1.01,1.15)$ \\
\hline $\mathrm{AUC}_{(0-\infty)}($ day $* \mu \mathrm{g} / \mathrm{mL})$ & $\mathrm{Al}$ & $478.94(0.026)$ & $449.70(0.026)$ & $1.07(1.00,1.13)$ \\
\hline
\end{tabular}

PFS or Al versus lyophilized product was assessed as an exploratory objective.

Results/Discussion: Geometric mean estimates were adjusted for injection site (arm, abdomen, and thigh) and baseline weight ( $\log _{e}$ scale). Al, prefilled autoinjector; $\mathrm{AUC}_{(\mathrm{O}-\mathrm{t})}$, area under the concentration-time curve from time zero (pre-dose) to last time of quantifiable concentration; $\mathrm{AUC}_{(\mathrm{O}-\infty)}$, area under the concentrationtime curve from time zero to infinity; $\mathrm{Cl}$, confidence interval; $\mathrm{C}_{\max }$, maximum plasma concentration; PFS, prefilled syringe; SE, standard error.

Results: In total, 244 participants received the study drug. Primary (Table) and secondary PK parameters were comparable across the three treatment groups; $90 \%$ confidence intervals for primary endpoint treatment ratios (PFS or Al versus lyophilized formulation) were all within the conventional bioequivalence bounds $(0.80,1.25)$. In all three treatment groups, mepolizumab exposure did not markedly differ across the three assessed sites of injection (upper arm, abdomen, or thigh). Across all three groups, the incidence of ontreatment adverse events was $29 \%-38 \%$; headache (9\%), viral upper respiratory tract infection (5\%), and fatigue (3\%) were the only ontreatment adverse events reported by $\geq 3 \%$ of participants. There was a low incidence (5\%) of anti-drug antibodies (none neutralizing) reported across the three groups. A similar effect on blood eosinophils was also observed across the three groups.

Conclusions: In this single-dose study, the PK profile of the mepolizumab liquid formulation administered via PFS or Al was statistically comparable to the approved lyophilized formulation. Its safety profile was consistent with that of the lyophilized formulation, with no safety concerns identified. Mepolizumab exposure did not markedly differ between injection sites. Treatments resulted in a similar effect on blood eosinophils.

\section{REFERENCE(S)}

This abstract was previously presented at ATS 2019, Am J Respir Crit Care Med. 2019;199:A1311

Funding: GSK (Study 204958; NCT03014674).

\section{P140 | First clinical study with AP30663, a small conductance calcium-activated potassium $\left(\mathrm{K}_{\mathrm{Ca}} 2\right)$ channel inhibitor developed for cardioversion of atrial fibrillation}

\author{
Pim Gal ${ }^{1}$; Nils Edvardsson ${ }^{2}$; Michiel Kemme ${ }^{3}$; Jacobus Burggraaf ${ }^{1}$; \\ Morten Grunnet ${ }^{2}$; Jonas Diness ${ }^{2}$ \\ ${ }^{1}$ Centre for Human Drug Research; ${ }^{2}$ Acesion Pharma Inc.; ${ }^{3}$ VU Medical \\ center
}

Introduction/Background \& Aims: Effective, safe, and tolerable pharmacological treatment for episodes of atrial fibrillation (AF) remains an unmet need. The small conductance $\mathrm{Ca}^{2+}$-activated $\mathrm{K}^{+}$channel, also known as $\mathrm{K}_{\mathrm{Ca}} 2$ or SK channel, has emerged as a potential new target for AF treatment. This first-in-human study evaluated the safety and tolerability of AP30663, a $\mathrm{K}_{\mathrm{Ca}} 2$-channel inhibitor, which prolonged the atrial effective refractory period and converted AF into sinus rhythm in preclinical studies. In addition, pharmacokinetics and the pharmacodynamic effects were explored.

Method/Summary of work: Forty-seven healthy male volunteers received a 30-minute intravenous administration of AP30663 in ascending doses, ranging from $1 \mathrm{mg} / \mathrm{kg}$ to $6 \mathrm{mg} / \mathrm{kg}$. Due to infusion site reactions, changes to the formulation and administration were implemented in the latter twenty-four volunteers. Extractions from a 24-hour continuous electrocardiogram (ECG) were used to evaluate the pharmacodynamic effect of AP30663, including QT subinterval analysis. The QT interval was corrected for heart rate using Fridericia's formula (QTcF). ECG data were analyzed with a repeated measures ANCOVA, and a noncompartmental analysis was used to compute the pharmacokinetic parameters.

Results/Discussion: Mean age was $23.7 \pm 3.0$ years. In total, 34 adverse events (AEs) considered related to AP30663 exposure were observed. All were local infusion site reactions, mild and temporary in nature, although full recovery took up to 110 days. After formulation and administration changes, local infusion site reactions still occurred, but the median duration dropped from 37 days in the $2 \mathrm{mg} / \mathrm{kg}$ dose level to 5 days in the $6 \mathrm{mg} / \mathrm{kg}$ dose level. There were no systemic AEs related to AP30663 administration. As was to be expected in healthy volunteers, no effect of AP30663 was observed 
LSMeans $[90 \% \mathrm{CI}]$
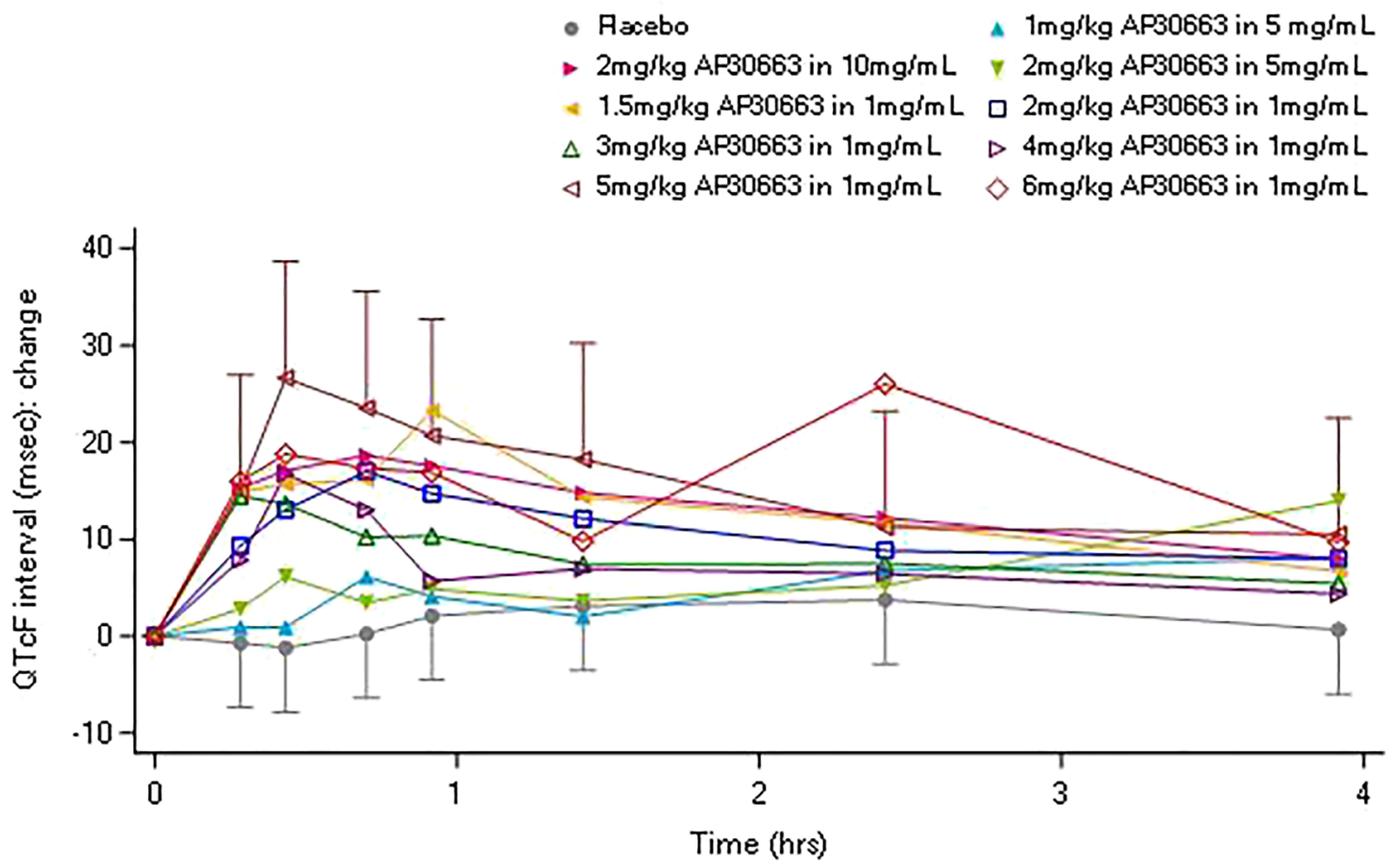

FIGURE 1. Effect of AP30663 on the QTcF interval

on atrial surface ECG parameters, RR-interval or QRS duration, but there was a dose-dependent effect on the QTcF interval of $+16.5 \mathrm{~ms}$ (baseline controlled difference with placebo, $P=0.025$ ) for the highest dose level (Figure 1). AP30663 dose-dependently increased both the corrected J point-T peak ( $\mathrm{Pp}-\mathrm{Tpc}$ ) and the Tpeak-Tend (Tp-Te) intervals. Mean clearance was $12.5 \pm 3.2 \mathrm{~L} / \mathrm{h}$ and tended to be higher for the two highest dose levels $(16.7 \mathrm{~L} / \mathrm{h})$ as compared to the lower dose levels $(12.3 \mathrm{~L} / \mathrm{h})$. Volume of distribution ranged from $65.8 \mathrm{~L}$ at the lowest dose to $98.4 \mathrm{~L}$ at the highest dose levels. The mean terminal half-life was $4.7 \pm 0.9$ hours. AP30663 displayed a less than dose proportional increase in Cmax, with the dose-normalized Cmax being about half in the $6 \mathrm{mg} / \mathrm{kg}$ dose level as compared to the dose normalized $\mathrm{Cmax}$ in the $1.5 \mathrm{mg} / \mathrm{kg}$ dose level.

This figure displays the effect of AP30663 on the QTcF interval in the first 4 hours after dose administration. Note that there is a dose-dependent effect of AP30663 on the QTcF interval.

Conclusion(s): Administration of AP30663, a novel $\mathrm{K}_{\mathrm{Ca}^{2}}$-channel inhibitor, was considered safe and well tolerated systemically. A mild increase in the QTcF interval was observed in the investigated dose levels, predominantly mediated through an increase in the Jp-Tpc and Tp-Tend intervals. These results support further development of AP30663.

\section{P141 | Activity and pulmonary function collected via a noninvasive platform differentiate healthy and asthmatic children}

Matthijs Kruizinga ${ }^{1}$; Nikki van der Heide ${ }^{1}$; Marianne Nuijsink ${ }^{2}$; Rik Stuurman ${ }^{1}$; Adam Cohen ${ }^{1}$; Gertjan Driessen ${ }^{2}$

${ }^{1}$ Centre for Human Drug Research; ${ }^{2}$ Juliana Children's Hospital

Introduction/Background \& Aims: Pediatric clinical trials are generally conducted in hospitals, and study data are collected during visits at predefined time intervals. Additional visits and invasive measurements are increasingly hard because of the increase of the burden for subjects. In the case of pediatric asthma, information about physical activity, pulmonary function, and patient-reported outcomes collected via a home monitoring platform (CHDR MORE ${ }^{\circledR}$ ) could allow for future trials to be conducted completely at home. The aim of this study was to investigate whether home monitoring of physical activity and pulmonary function could discriminate healthy and asthmatic children and to correlate home-measured physical activity and FEV1 with existing clinical endpoints.

Method/Summary of work: Healthy subjects and patients aged 6-16 with either controlled or uncontrolled (based on GINA criteria and Asthma Control Questionnaire) asthma were recruited. Patients were monitored for 4 weeks with a smartwatch (Withings Steel HR) that registered physical activity (step count), heart rate, and sleep. Patients also performed daily pulmonary function tests (PFTs; NuvoAir AirNext) and completed a daily symptom diary (Asthma Control 
Diary). Healthy subjects were monitored for 3 weeks with the Steel HR and performed biweekly PFTs. The difference in physical activity between the three study groups was assessed via a mixed model correcting for age, gender, daily rain duration, and school days. Repeated measurements correlations were performed to investigate correlations between physical activity, FEV1, and symptom diary score.

Results/Discussion: 128 subjects (30 with controlled asthma, 21 with uncontrolled asthma, and 77 healthy controls) were included. Subjects with uncontrolled and controlled asthma had a significantly lower physical activity level than healthy subjects. Asthma diary symptom score showed a negative correlation with daily physical activity for subjects with uncontrolled asthma $\left(R_{R M}-0.21, p<0.001\right)$ but not for subjects with controlled asthma $\left(R_{R M} 0.04, p=0.27\right.$ ) (Figure 1). Furthermore, daily FEV1 was associated with the asthma diary symptom score for patients with uncontrolled asthma $\left(R_{R M}-0.23, p<0.001\right)$ and controlled asthma $\left(\mathrm{R}_{\mathrm{RM}}-0.1, p=0.01\right)$.

Conclusion(s): We demonstrated that physical activity can discriminate between healthy children and children with (un)controlled asthma. Furthermore, we have shown correlations between physical activity, home-measured spirometry, and traditional endpoints. We propose physical activity is a potential new clinical endpoint, but more advanced analyses, as well as a longer follow-up period, are necessary to substantiate these results.

\section{P142 | IL6 \& CRP: Association with immunological, virological, and lipid status in paediatric HIV individuals on efavirenz-based anti-retroviral therapy}

Purba Chakrabarty ${ }^{1}$; Manasi Basu Banerjee ${ }^{1}$; Manab Nandy ${ }^{1}$; Kalpana Datta ${ }^{1}$; Mitali Basu ${ }^{2}$; Shatavisa Mukherjee ${ }^{3}$

${ }^{1}$ Medical College and Hospital, Kolkata, West Bengal, India; ${ }^{2}$ Bankura Sammilani Medical College and Hospital, Bankura; ${ }^{3}$ School of Tropical Medicine, Kolkata

Introduction/Background \& Aims: Advances in HIV management and advent of anti-retroviral therapy (ART) has ensued a prolonged survival in HIV-infected individuals. Disease-related complications and adverse drug reactions (ADRs) can however compromise their quality of life, which needs management and periodic monitoring. Both HIV and ART are associated with lipid derangements and are significant contributors of cardiovascular disease (CVD). Persistent inflammation could be noticed even in treated patients attributing to modifications of lipid composition and function, further adding up to the exacerbating CVD risk. Inflammatory markers like CRP and IL-6 may highlight this risk prior. Efavirenz is an important component for first line ART, many of which primely contribute to lipid abnormalities. The present study aimed to probe the association between these inflammatory biomarkers and alterations in immunological, virological, and lipid profile in paediatric HIV individuals on efavirenz-based ART.
Method/Summary of work: This longitudinal study included paediatric HIV 1 patients, who were followed up for lipid profile alteration along with virological and immunological status at baseline and specific intervals till 6 months of therapy initiation. Study commenced after obtainment of ethical clearance and informed consent was ensured prior subject inclusion. Results were statistically analysed.

Results/Discussion: Subject population showed equal distribution of gender and ART regimens. $47.62 \%$ and $21.43 \%$ patients presented with elevated IL6 [Median (IQR) $=14.60$ (02.00-39.60)] and CRP $[$ Median (IQR) $=03.00(03.00-03.35)]$ levels respectively after 6 months of therapy initiation. Peripheral viral load (PVL) level was seen after 6 months of therapy initiation along with noting of baseline CD4 level. Out of those with elevated IL6, 55\% had presented with altered lipid parameters. Significant association had been found with regimens and lipid profile alterations. Significant association (Kappa value $=0.883, p$ value $=0.000$ ) has been found between the $<500$ baseline CD4 and elevated IL6 level; and elevated PVL and elevated IL6 level (Kappa value $=0.417, p$ value $=0.003$ )

Conclusion(s): Studies are needed to probe into the complex CVD pathogenesis in HIV-positive patients in order to determine whether therapies lowering these inflammatory biomarkers can reduce impending CVD risk. Thus, in future mankind can come up with better ART regimens with more anti-inflammatory and less lipid profile deranging properties.

\section{REFERENCE(S)}

1. Miller TL, Somarriba G, Orav EJ, Mendez AJ, Neri D, Schaefer N, et al. Biomarkers of vascular dysfunction in children infected with human immunodeficiency virus-1. J Acquir Immune Defic Syndr. 2010 Oct 1;55 (2):182-8.

\section{P143 | SAMe- $\mathrm{TT}_{2} \mathbf{R}_{\mathbf{2}}$ score and prediction of anticoagulation control with warfarin in Arab patients with a mechanical heart valve prosthesis}

Azra Mahmud; Reem Sirriyeh; Samir Shnaikat; Samiah Refaei; Fadi Obaid; Muayed Al-Zaibag

King Abdul Aziz Medical City

Introduction/Background \& Aims: The efficacy and safety of warfarin therapy for stroke prevention in mechanical heart valve (MHV) patients depends upon the time in therapeutic range (TTR). We aimed to assess the predictive ability of SAMe- $\mathrm{TT}_{2} \mathrm{R}_{2}$ score in Arab mechanical heart valve patients, where the choice of anti-coagulation is only warfarin.

Method/Summary of work: This is a single center retrospective study conducted in the anti-coagulation Clinic at King Abdul Aziz Cardiac Center, Riyadh, Saudi Arabia, in patients of Arab descent. Patients with MHV on warfarin between the year 2012 and 2015 were stratified according to SAMe-TT $\mathrm{R}_{2}$ score, and TTR was calculated using the Rosendaal method with target INR 2.0-3.0 for 
aortic and 2.5-3.5 for mitral MHV, in accordance with International Guidelines. The predictive power of $\mathrm{SAMe}-\mathrm{TT}_{2} \mathrm{R}_{2}$ score to predict TTR $<70 \%$ was assessed. The data were analysed using JMP Pro, Version 13(SAS for Windows).

Results/Discussion: The study sample included $270 \mathrm{MHV}$ patients on warfarin (mean age $54 \pm 0.81$ years, $46 \%$ female). The minimum SAMe- $\mathrm{TT}_{2} \mathrm{R}_{2}$ score was $\geq 2$ in all patients. The mean and median TTR were $65 \pm 0.1$ and $64 \%$ (interquartile range: $12.5 \%$ and $100 \%$ ) respectively. There was a significant linear relationship between the SAMe-TT ${ }_{2} \mathrm{R}_{2}$ score and the INR target; $45 \%$ of patients with an INR target of 2.5-3.5 had a score $>4$ vs. $33 \%$ with the lower target of 2-3 ( $p<0.0001)$. The low-risk group (score $<2$ ) had a significantly higher TTR as compared with the high-risk group (score $>2$ ), $71 \%$ vs. $63 \%, p=0.004$. Similarly, the percentage of patients with TTR $\geq 60 \%$, $65 \%$, or $70 \%$ was higher in the low-risk group ( $p=0.006, p=0.002$, and $p=0.003$, respectively) compared with the high-risk group. When assessing the ability of the SAMe$\mathrm{TT}_{2} \mathrm{R}_{2}$ score to predict the outcome of TTR $<70 \%$ using the ROC curve, the cutoff point $>2$ showed the best combination of sensitivity and specificity ( $81 \%$ and $64 \%$, respectively). The area under the curve was 0.60 ( $95 \% \mathrm{Cl}: 0.55-0.671 ; p=0.006$ ). The SAMe$\mathrm{TT}_{2} \mathrm{R}_{2} \geq 3$ did not show any additional predictive power compared with a score $>2$.

Conclusion(s): Our study shows that race is the most significant factor in determining TTR and a higher SAMe-TT ${ }_{2} \mathrm{R}_{2}$ does not provide any additional predictive value in Arab MHV patients.

\section{P144 | Assessment of blood pressure control and target organ damage in a treated hypertensive population-Is clinic BP measurement enough?}

Azra Mahmud; Ruba Alahaideb; Haifa Alshammary; Afnan Alfawwaz; Mayar Abanumay; Muyaed Al-Zaibag

King Abdul Aziz Medical City

Introduction/Background \& Aims: Hypertension is the leading risk factor for cardiovascular (CV) disease and a major contributor to morbidity, mortality, and disability worldwide. Hypertension is also a major CV risk factor in Saudi Arabia, with a prevalence of some $26 \%$ in the general population but higher in established CV disease, ranging between $45 \%$ and $75 \%$. Despite a large repertoire of antihypertensive drugs available, blood pressure (BP) control rates are still suboptimal.

Recent studies have shown that clinic BP alone may be a poor guide to hypertension control. Ambulatory blood pressure (ABPM) is the gold standard for diagnosis of hypertension, assessment of BP control, and its various patterns may provide prognostic information beyond clinic BP.
Therefore, the primary aim of our study was to compare BP control with clinic versus ABPM criteria. The secondary aim was to study the relationship between various ABPM patterns and subclinical target organ damage, ie, urine albumin-creatinine ratio (ACR), and arterial stiffness as pulse wave velocity (PWV) and biomarkers including high-sensitivity $\mathrm{C}$-reactive protein (hs-CRP) and brain natriuretic peptide (BNP) in a Saudi hypertensive population.

Method/Summary of work: We studied consecutive patients aged 18-80 years old referred to the Hypertension Clinic at King Abdul Aziz Cardiac Center, Riyadh, Saudi Arabia, between January 2016 and December 2018. ABPM was performed for a 24-hour period with an oscillometric device (Spacelabs), with readings every 30 minutes from $6 \mathrm{AM}$ to $10 \mathrm{PM}$, and every 60 minutes from $10 \mathrm{PM}$ to 6 AM. Patient diary was used to define sleep/wake periods. White coat effect was defined as office systolic blood pressure (SBP) $\geq 140 \mathrm{mmHg}$ or diastolic blood pressure (DBP) $\geq 90 \mathrm{mmHg}$ with daytime ABPM SBP $<135 \mathrm{mmHg}$ and DBP $<85 \mathrm{mmHg}$. Uncontrolled clinic BP was defined as systolic $>140 \mathrm{mmHg}$ and diastolic $\mathrm{BP}>90 \mathrm{mmHg}$. Masked uncontrolled hypertension (MUCH) was defined as controlled clinic BP with daytime BP $\geq 135 / 85 \mathrm{mmHg}$ (definition-1); 24-hour ambulatory $\mathrm{BP} \geq 130 / 80 \mathrm{mmHg}$ (definition2); and either daytime $\mathrm{BP} \geq 135 / 85 \mathrm{mmHg}$ or nighttime BP $\geq 120 / 70 \mathrm{mmHg}$ (definition-3). Dipping was defined as a $10-20 \%$ fall in nocturnal BP; non-dipping as $<10 \%$, reverse-dipping as $\leq 0 \%$, and extreme dipping as $>20 \%$. Arterial stiffness was measured as PWV using SphygmoCor (AtCor Medical, Australia) and Mobilograph (IEM Medical). Hs-CRP, BNP, and ACR were measured using standard laboratory investigations. Results analysed using JMP Version 13, $p<0.05$ considered significant. The study had Institutional Ethics Committee permission.

Results/Discussion: The study population included 428 subjects (mean age $53.5 \pm 14$ years, female $=45 \%$ ). Nine percent of the population had WC; $45 \%$ had uncontrolled hypertension using clinic BP compared with ABPM criteria; $24 \mathrm{hr}, 68 \%$; daytime, 56\%; and nighttime, $86 \%$ ( $p<0.0001)$. Overall, $71 \%$ of the patients had MUCH which was driven by nocturnal hypertension vs. $24 \mathrm{hr}$ or daytime $(70 \%$ vs. $42 \%$ vs. $32 \%, p<0.0001)$ respectively. Seventy-nine percent of the cohort had non-dipping of which $45 \%$ were reverse dippers; $6 \%$ extreme dippers and $18 \%$ dippers. Patients with ambulatory hypertension had significantly higher ACR (30.8 \pm 8 vs. $6.2 \pm 6, p<0.01)$, PWV (11.2 vs. 8.9, $p<0.001)$, BNP (1.2 \pm 1 vs. $6.5 \pm 1, p<0.05)$, and hs$\operatorname{CRP}(1.6 \pm 1.4$ vs. $6.4 \pm 2.3, p<0.01)$ compared with those with raised clinic BP alone.

Conclusion(s): The prevalence of masked suboptimal BP control in Saudi patients with treated and well-controlled clinic BP is high and is largely because of nocturnal hypertension. Ambulatory hypertension is also characterized by sub-clinical target organ damage, arterial stiffness, and vascular inflammation, not related with clinic BP. Therefore, ABPM should become a routine investigation to confirm BP control, especially in higher risk groups and/or those with borderline hypertension. 


\section{P145 | Comparison of pharmacotherapy competence of good and poor prescribers in primary care}

Omer Atac ${ }^{1}$; Volkan Aydin ${ }^{2}$; Ahmet Akici ${ }^{2}$

${ }^{1}$ Department of Public Health, School of Medicine, Istanbul Medipol University, Istanbul, Turkey; ${ }^{2}$ Department of Medical Pharmacology, School of Medicine, Marmara University, Istanbul, Turkey

Introduction/Background \& Aims: Pharmacotherapy is an important part of the primary care practice which was undertaken by family physicians. The extent of rationality for this health care practice needs to elucidated. In this study, we aimed to compare the rationality of treatment of frequently encountered acute and chronic indications based on the prescribing performance of primary care physicians.

Method/Summary of work: Prescriptions which have single diagnosis ( $n=2,947,112$ ) of 1052 family physicians in 2016 selected by systematic sampling in Istanbul were included in the study. According to the evaluation of the number of drugs and antibiotics per prescription and the cost of drugs per prescription, the physicians in the lowest and highest quartiles were classified as poor prescribers $(n=227)$ and good prescribers $(n=210$ ), respectively. The pharmacotherapy approaches of these physician groups in tonsillopharyngitis and essential hypertension, which were determined as the most common single acute and chronic indications, respectively, were compared in terms of compliance with relevant treatment guidelines.

Results/Discussion: Hypertension and tonsillopharyngitis single diagnoses constituted 9.5\% $(n=280,772)$ and $6.6 \%(n=194,530)$ of all the examined prescriptions, respectively. The compliance of hypertension prescriptions with treatment guidelines was detected as higher in good prescribers $(40.8 \%)$ than that in poor prescribers (34.8\%) $(p<0.001)$. We found the number of encounters containing the contraindicated drugs in hypertension as 232 (0.5\%) in good prescribers and $478(0.8 \%)$ in poor prescribers, where the difference was significant $(p<0.001)$. The compliance of tonsillopharyngitis prescriptions with treatment guidelines was found to be higher in good prescribers (57.9\%) compared to that in poor prescribers $(50.7 \%)(p<0.001)$. The number of tonsillopharyngitis prescriptions containing inappropriate drugs for this indication was $36(0.08 \%)$ among good prescribers and $96(0.3 \%)$ among poor prescribers in this diagnosis $(p<0.001)$.

Conclusion(s): This study reveals that the rational pharmacotherapy competences of primary care physicians lag behind expectations in general, especially in poor prescribers. It is understood that physicians who are more irrational in overall prescription performance tend to exhibit also more irrational behaviors regarding the management of hypertension and tonsillopharyngitis. It is thought that while developing strategies for disseminating rational drug use, this situation may help to guide in better planning of the details of the physician performances and indication selection for physician-centered interventions.

\section{P147 | Lipocalin-2 variants as effective biomarkers to stratify patients with heart failure and/or chronic kidney disease}

\author{
Dahui Li ${ }^{1}$; Wai Yan Sun ${ }^{1}$; Aimin $\mathrm{Xu}^{1}$; Junwei Yang ${ }^{2}$; G.R. De Mey Jo ${ }^{3}$; \\ Yu Wang ${ }^{1}$ \\ ${ }^{1}$ The University of Hong Kong; ${ }^{2}$ Second Affiliated Hospital, Nanjing \\ Medical University; ${ }^{3}$ University of Southern Denmark
}

Introduction/Background \& Aims: Lipocalin-2 has been regarded as a sensitive biomarker for acute kidney injury (AKI). Its levels predict the development of chronic kidney disease (CKD) and the mortality of heart failure (HF). The present study evaluated whether measuring different variants of lipocalin-2 represents a better approach for stratifying patients with CKD or HF.

Method/Summary of work: In-house immunoassays were developed to quantify three variants of human lipocalin-2, hLcn2, C87A, and R81E. The lipocalin-2 variants were measured in samples from human cohorts, including 130 healthy volunteers, 104 CKD, and $160 \mathrm{HF}$ patients. The distribution of lipocalin-2 levels was analyzed against various parameters of cardiac and renal functions.

Results/Discussion: Compared to the healthy cohort, serum C87A levels were significantly increased in patients with CKD and negatively correlated with estimated glomerular filtration rate (eGFR), positively correlated with blood urea nitrogen (BUN) and serum creatinine (Scr), parameters associated with kidney function. Both C87A and R81E levels increased, whereas hLcn2 decreased in the urine samples of CKD patients. The urinary R81E levels were significantly correlated with eGFR, BUN, and Scr. The serum to urine ratio of R81E represented a better parameter than hLcn2 and C87A for stratifying CKD patients. In HF patients, the plasma and pericardial fluid levels of hLcn2 and C87A, but not R81E, were significantly correlated. After adjustment for age, gender, smoking, body mass index (BMI), BUN, and Scr, the plasma levels of C87A and the ratio of C87A to brain natriuretic peptide (BNP)were significantly correlated with left ventricular ejection fraction (LVEF).

Conclusion(s): Simultaneously quantifying different lipocalin-2 variants represents a promising approach to stratify patients with CKD and/or HF.

\section{P148 | Occult hepatic steatosis-How can we distinguish true treatment emerging liver signals from undetected unrelated liver abnormalities?}

Richard Francis-Sakai; Tomasz Pierscionek; Simon Coates; Jorg Taubel

Richmond Pharmacology 
Introduction/Background \& Aims: Hepatic steatosis is a largely benign condition affecting up to $25 \%$ of the European population. ${ }^{[1]} \mathrm{A}$ correlation between fat deposition in the liver and elevated serum alanine transaminase (ALT) is recognised ${ }^{[2]}$ and could lead to steatohepatitis. ${ }^{[2]}$ There is evidence that hepatic steatosis and steatohepatitis can affect drug metabolism. ${ }^{[3]}$ Including volunteers with liver abnormalities could impact data quality and lead to variability in pharmacokinetic data or derangement of LFTs being misattributed solely to the drug being tested. This may pose a potential risk to volunteer safety through increased risk of toxicity and adverse effects. It is therefore desirable to find screening tests which more reliably identify hepatic steatosis.

Method/Summary of work: We conducted a prospective longitudinal study of healthy Caucasian and Japanese male volunteers. Measurements of liver function (ALT, AST, prothrombin time, and albumin), body weight, BMI, and liver ultrasound scans were collected.

Results/Discussion: From a sample of 45 volunteers, 15 (33\%) were found to have some degree of hepatic steatosis, all of whom had normal blood results and stable weight/BMI. Two volunteers (out of 45) with normal ultrasound scans had serum ALT readings of up to twice the upper limit of normal. The results of ultrasound scans of additional volunteers with intermittent transaminase elevations are pending at the time of writing this abstract but will be presented in the poster. Conclusion(s): No clear correlation was found between weight/BMI or liver function tests and radiological evidence of hepatic steatosis. The presence of hepatic steatosis in a significant proportion of healthy volunteers with normal BMI and LFT readings suggests that current measures to ensure no volunteer with hepatic steatosis enters a clinical trial may be inadequate. To reduce the need for ultrasound scans on all prospective trial volunteers, the identification of predictive factors for hepatic steatosis is desirable. Further research will include studying more volunteers (>200) and examining a wider range of variables (lifestyle, demographics, past medical history, and blood tests) to identify potential predictive factors.

\section{REFERENCE(S)}

1. Andronescu, C. I., Purcarea, M. R., \& Babes, P. A. (2018) Nonalcoholic fatty liver disease: epidemiology, pathogenesis and therapeutic implications. Journal of medicine and life, 11(1), 20-23.

2. Sattar, N., Forrest, E., \& Preiss, D. (2014) Non-alcoholic fatty liver disease. BMJ (Clinical research ed.), 349, g4596.

3. Merrell, M. D., \& Cherrington, N. J. (2011) Drug metabolism alterations in nonalcoholic fatty liver disease. Drug metabolism reviews, 43(3), 317-334.

\section{P149 | The effects of different statins on serum LDL cholesterol concentrations: A systematic review and meta-analysis of in vivo dose-response curves}

Genevieve Monaghan ${ }^{1}$; Richard Stevens ${ }^{2}$; Jeffrey Aronson ${ }^{3}$

${ }^{1}$ University of Birmingham; ${ }^{2}$ University of Oxford; ${ }^{3}$ Green-Templeton

College, Oxford

Introduction/Background \& Aims: Dosage regimens should optimize the balance between the chance of achieving desired benefits and the desire to avoid possible harms. Dose-response curves for both benefits and harms form the foundations for devising such regimens. We have generated dose-response curves for the effects of different statins on serum LDL cholesterol concentrations from a systematic review and meta-analysis of in vivo data from multi-arm statin trials published in clinicaltrials.gov. This was a student project by the firstnamed author.

Method/Summary of work: We searched clinicaltrials.gov for trials in which changes in LDL concentrations had been measured in patients taking different dosages of statins. We excluded one-arm and nonrandomized studies; studies that did not measure LDL or non-HDL cholesterol before and after treatment with the statin; studies that did not compare multiple dosages of the statin or compare the statin with a placebo or control group; studies in which the statin was taken for less than 2 weeks; studies, or arms of studies, in which the statin was used in combination with another lipid-modifying drug; and studies in which the dosage of the statin was titrated or changed during the trial or when a range of dosages was allowed in a study arm. We fitted the data to a sigmoid $\mathrm{E}_{\max }$ model, using network meta-analysis, fixing the Hill coefficient at 1. We used R software (version R-3.0.2, http:// www.r-project.org) for the analyses, as previously described [1]

Results/Discussion: The results are shown in Table 1.

Conclusion(s): Our calculations of $\mathrm{ED}_{50}$ values are limited by the fact that few studies have included low daily doses (that is, doses below the calculated $\mathrm{ED}_{50}$ ). However, these results suggest that statins have generally been studied and are currently being used in high or moderately high daily doses. Our results also suggest that increasing the daily dose of atorvastatin from $20 \mathrm{mg}$ to $80 \mathrm{mg}$, as is recommended after a first cardiovascular event, should not be considered a change from a low dose to a high dose, since both are well above the $\mathrm{ED}_{50}$; in such cases, adverse effects, such as muscle pain, are more likely to 
TABLE 1 Values of $\mathrm{ED}_{50}$ and $\mathrm{E}_{\max }$ computed from published trial data along with usual dosage recommendations as published in manufacturers' literature

\begin{tabular}{|lllllr|}
\hline Statin & No. of trials & $\begin{array}{l}\text { Dosages studied } \\
\text { (mg/day) }\end{array}$ & ED $_{50}$ (mg/day) & $\begin{array}{l}E_{\text {max }} \text { (\% reduction in } \\
\text { LDL cholesterol) }\end{array}$ & $\begin{array}{l}\text { Recommended dosage } \\
\text { range (mg/day) }\end{array}$ \\
\hline Pitavastatin & 4 & $1,2,4$ & 1.78 & 40 & $2-4$ \\
\hline Atorvastatin & 55 & $5,10,20,40,80$ & 4.61 & 53 & $10-80$ \\
\hline Pravastatin & 11 & $10,20,40,80$ & 11.44 & 33 & $10-40$ \\
\hline Simvastatin & 20 & $20,40,80$ & 11.75 & 51 & $10-80$ \\
\hline
\end{tabular}

occur with little additional benefit. Future work should include similar analyses of dose-response curves for harms, for comparison with the curves for benefit, in order to determine dosage regimens that optimize the benefit to harm balance.

\section{REFERENCE(S)}

1. Langford O, Aronson JK, van Valkenhoef G, Stevens RJ. Methods for meta-analysis of pharmacodynamic dose-response data with application to multi-arm studies of alogliptin. Stat Methods Med Res $2016 \mathrm{Mar}$ 17. pii: 0962280216637093

\section{P152 | Prescribing of psychotropic medications in individuals with anorexia nervosa in UK primary care, 1996-2016: A retrospective database study}

\author{
Maedeh Y. Beykloo ${ }^{1}$; Ruth Brauer ${ }^{1}$; Elizabeth Mills ${ }^{1}$; Ian Wong ${ }^{2}$ \\ ${ }^{1}$ University College London (UCL); ${ }^{2}$ University of Hong Kong
}

Introduction/Background \& Aims: There's a call for greater understanding of psychotropic prescribing in individuals with anorexia nervosa (AN) within the United Kingdom (UK). This study aimed to comprehensively assess trends in the incidence and prevalence of AN and the prescribing of psychotropic medications in individuals with AN over a 20 year period.

Method/Summary of work: A retrospective database study was conducted using anonymised medical records from general practices throughout the UK. Individuals aged 12-50 years and with recorded AN diagnosis were identified between 1996 and 2016. The incidence and prevalence of AN were calculated, and Kaplan Meier analyses were used to assess the initiation and discontinuation of psychotropic prescribing in this cohort.

Results/Discussion: The incidence of AN remained fairly constant for females and males. The prevalence of AN decreased from $80.74 / 100,000$ person years in 1996 to $45.39 / 100,000$ person years in 2006 to $23.71 / 100,000$ person years in 2016, presenting a downward trend. $47.44 \%$ of individuals with AN received a prescription for psychotropic medication, with the most common antidepressant or antipsychotic prescribed being fluoxetine $(59.73 \%)$ and olanzapine (8.90\%) respectively. $18.36 \%$ of individuals received a first prescription in the first 6 months of AN diagnosis, with females being significantly more likely to receive a prescription any time after diagnosis compared to males (log-rank $p<0.001$ ).

Conclusion(s): Our findings in this study highlight the use of psychotropic medications for the treatment of AN despite lack of sufficient evidence. Our study calls for greater investigations into the benefits and harms associated with psychotropic medications in this population.

\section{P154 | Baseline data and treatment responses in continent and incontinent patients with overactive bladder syndrome}

\author{
Ayhanim Elif Muderrisoglu ${ }^{1}$; Sandra Murgas ${ }^{2}$; Tim Schneider ${ }^{3}$; \\ Matthias Oelke ${ }^{4}$; Martin Michel ${ }^{5}$ \\ ${ }^{1}$ Ankara University School of Pharmacy Dept. of Pharmacology; \\ ${ }^{2}$ Apogepha; ${ }^{3}$ Praxiskliniken Urologie Rhein-Ruhr; ${ }^{4}$ Dept. Of Urology, St, \\ Antonius Hospital; ${ }^{5}$ Dept. of Pharmacology, Johannes Gutenberg \\ University
}

Introduction: The overactive bladder syndrome $(O A B)$ is defined by the presence of urinary urgency, whereas urinary incontinence is a frequent but not mandatory part of the condition. A previous study found that baseline symptoms and improvements upon treatment were similar between continent and incontinent $O A B$ patients [1]. Therefore, we have compared baseline data and treatment responses of continent and incontinent $O A B$ patients based on data from two other previously reported studies [2].

Methods: Data from two non-interventional studies of similar design were analyzed [2]. Briefly, the symptoms of urinary incontinence, urgency, micturition frequency, and nocturia as well as the Patient Perception of Bladder Condition symptom score (PPBC) were systematically captured in OAB patients who were treated with the muscarinic receptor antagonist propiverine ER (30-45 mg/day; dose adjustment during the study was permitted). Because of the nonGaussian distribution of these parameters [2], data are shown as medians with inter-quartile ranges (IQR). In contrast, \% changes of $\mathrm{OAB}$ symptoms upon treatment are shown as means \pm SD because these do not exhibit major deviations from a normal distribution.

Results: Study 1 included 364 (median age: 66 years, 52\% female) continent and 787 (median age: 70 years, $71 \%$ female) incontinent 
patients, respectively. Study 2 included 171 (median age: 66 years, 57\% female) continent and 418 (median age: 69 years, 80\% female) incontinent patients, respectively. Consistent across both studies, incontinent patients were slightly older (3-4 years) and more likely to be female; they also exhibited a slightly greater symptom intensity at baseline, but differences were small (Table 1). In contrast, improvements of symptoms upon 12 weeks of treatment were similar in both groups (Table 2).

Conclusions: The present findings differ from our previous study [1] because baseline symptoms were somewhat greater in incontinent than in continent patients. However, consistent across all three studies, treatment responses were similar in both groups. We conclude that muscarinic antagonists such as propiverine are similarly effective in continent and incontinent $\mathrm{OAB}$ patients.

\section{REFERENCE(S)}

1. Michel MC, de la Rosette JJMCH, Piro M and Schneider T (2005) Comparison of symptom severity and treatment response in patients with incontinent and continent overactive bladder. Eur Urol 48:110-115.

2. Amiri M, Murgas S, Michel MC (2018) Do overactive bladder symptoms exhibit a Gaussian distribution? Implications for reporting of clinical trial data. Neurourol Urodyn 37 Suppl 5: S397-S398.

\section{P155 | Point prevalence survey on the antimicrobial use and healthcare-associated infections in a tertiary care centre in a developing country}

\author{
Aditi Panditrao ${ }^{1}$; Nusrat Shafiq ${ }^{1}$; Bhaskar Krishnamurthy ${ }^{2}$; \\ Manisha Biswal ${ }^{1}$; Ashish Kakkar ${ }^{1}$; Shweta Talati ${ }^{1}$ \\ ${ }^{1}$ Post Graduate Institute of Medical Education \& Research; ${ }^{2}$ Dr. Reddy's \\ Laboratories
}

Introduction/Background \& Aims: Point prevalence surveys (PPSs) have been developed and validated as a standardised method to collect and analyse antimicrobial use data at a hospital level. However, in the absence of electronic medical record systems and lack of dedicated resources, undertaking such a study in a tertiary care centre with a high patient load in resource poor settings can be challenging. We piloted this PPS to see feasibility of this exercise in a tertiary care centre in a developing country. This would enable other healthcare set-ups with similar resources in analysing the antimicrobial use at an institutional level to subsequently evaluate the temporal patterns of rational antimicrobial utilisation.

Method/Summary of work: This inpatient point prevalence survey was conducted in a tertiary care referral centre in a city in North India over 2 weeks in the month of September 2018. It covered 1910 beds in four subcentres-the main hospital, advanced paediatric centre, advanced trauma centre, and the advanced cardiac centre, excluding the outpatient and emergency departments. Teams including separate personnel for the antimicrobial audit and for infection control practices extracted data from bedside medical records in a predesigned proforma. Data from one unit were completely recorded in one day. Designated antimicrobials were colistin, amphotericin B, linezolid, tigecycline, caspofungin, minocycline, polymyxin $B$, anidulafungin, micafungin, and daptomycin

Results/Discussion: Of the 1171 total admitted patients, a total of 1631 antimicrobials were used in 712 patients (60.8\%), yielding 2.29 antimicrobials per patient. Of these, 1068 (66.4\%) were used empirically, 362 (22.5\%) prophylactic and 179 (11.1\%) were based on culture reports. There were 56 (7.9\%) instances of double anaerobic cover and 13 (1.8\%) instances of double cover for gram negative infections. 68 instances of escalation were observed, the most common antimicrobial being escalated to being colistin (11). 114 (9.7\%) healthcareassociated infections (HAl) were observed, bloodstream infection (44.7\%) being the commonest. The five most commonly isolated organisms from all cultures were Escherichia coli (9\%), Pseudomonas aeruginosa (4.5\%), Klebsiella pneumoniae (3.8\%), Acinetobacter baumannii (3.8\%), and Staphylococcus aureus (3\%)

Conclusion(s): This study demonstrates a feasible and effective method to undertake PPS in healthcare set-ups lacking electronic medical record systems with high patient load. Use of broad spectrum and designated antimicrobials was high, although explainable, in view of referred seriously ill patients with suspected or proven multidrug pathogens. Tailored antimicrobial stewardship and infection control

TABLE 1 The five most commonly used antimicrobials used

\begin{tabular}{|c|c|c|}
\hline Antimicrobial & $\begin{array}{l}\text { Total no. of } \\
\text { antimicrobials } \\
\text { used }\end{array}$ & $\begin{array}{l}\text { Percentage (\%) } \\
\text { of total } \\
\text { antimicrobials }\end{array}$ \\
\hline Cefuroxime & 151 & 9.3 \\
\hline $\begin{array}{l}\text { Piperacillin + } \\
\text { tazobactam }\end{array}$ & 138 & 8.5 \\
\hline Amikacin & 129 & 7.9 \\
\hline Ceftriaxone & 109 & 6.7 \\
\hline Vancomycin & 103 & 6.3 \\
\hline
\end{tabular}

TABLE 2 Total use of designated antimicrobials

\begin{tabular}{|c|c|c|}
\hline Antimicrobial & $\begin{array}{l}\text { Total no. of } \\
\text { antimicrobials } \\
\text { used }\end{array}$ & $\begin{array}{l}\text { Percentage (\%) } \\
\text { of total } \\
\text { antimicrobials }\end{array}$ \\
\hline Colistin & 66 & 4 \\
\hline Amphotericin B & 44 & 2.7 \\
\hline Linezolid & 14 & 0.8 \\
\hline Tigecycline & 8 & 0.5 \\
\hline Caspofungin & 5 & 0.3 \\
\hline Minocycline & 3 & 0.2 \\
\hline Polymyxin B & 2 & 0.1 \\
\hline Anidulafungin & 2 & 0.1 \\
\hline Micafungin & 1 & 0.06 \\
\hline Daptomycin & 0 & 0 \\
\hline
\end{tabular}


interventions will be needed to reduce the need for use of antimicrobials in the future

\section{P156 | Medication regimens can be SIMPLER: Outcomes of a cluster randomised controlled trial in 8 Australian residential aged care facilities}

\author{
Janet Sluggett ${ }^{1}$; Esa Chen ${ }^{1}$; Jenni Ilomaki ${ }^{1}$; Megan Corlis ${ }^{2}$; \\ Sarah Hilmer ${ }^{3}$; J. Simon Bell ${ }^{1}$ \\ ${ }^{1}$ Monash University; ${ }^{2}$ Helping Hand Aged Care; ${ }^{3}$ Australia
}

Introduction/Background \& Aims: Complex medication regimens are burdensome for residents and are associated with medication administration errors and poor health outcomes in residential aged care facilities (RACFs). The SImplification of Medications Prescribed to Long-tErm care Residents (SIMPLER) study assessed the application of a structured process to consolidate the number of medication administration times for residents.

Method/Summary of work: We undertook a non-blinded, matchedpair, cluster randomised controlled trial of a single intervention delivered at the individual resident level. The trial was registered with the Australian New Zealand Clinical Trials Registry (ACTRN12617001060336) and the protocol has been published [1]. Trained research nurses recruited permanent residents who were English-speaking and charted at least 1 medication regularly. The intervention involved a clinical pharmacist applying a validated 5-step tool to assess medications charted for regular administration and identify opportunities simplify medication use (e.g. by administering medications at the same time or using longer-acting or combination formulations). Residents in the comparison group received routine care. The primary outcome was the number of medication administration times per day for medications charted regularly at 4 months post-study entry. Secondary outcomes included resident satisfaction, quality of life, falls, incidents, hospitalisations, and mortality. Associations between the intervention and study outcomes were estimated using linear mixed models with a constrained longitudinal data analysis approach, with RACF included as a random effect.

Results/Discussion: 99 residents participated in the intervention arm, and 143 were recruited in the comparison RACFs. The mean age of residents was 86 years (standard deviation [SD] 8.1), and $74 \%$ were female. The mean number of medication administration times per day at baseline was 4 (SD 1.7). Medication simplification was possible for 62 (65\%) of the 96 residents who received the intervention, and 57 (62\%) of the 92 simplification recommendations were implemented at follow-up. At follow-up, the mean number of administration times was reduced in the intervention arm in comparison to usual care $(-0.36,95 \%$ confidence interval -0.63 to $-0.09, p=0.01$ ).
Conclusion(s): Single application of a structured, validated tool to reduce regimen complexity is a low-risk intervention and reduces the burden of medication taking among residents of aged care facilities.

\section{REFERENCE(S)}

1. Sluggett JK, Chen EYH, llomäki J, Corlis M, Hilmer SN, Van Emden J, Ooi CE, Nguyen KH, Comans T, Hogan M, Caporale T, Edwards S, Quirke L, Patching A, Bell JS (2018) SImplification of Medications Prescribed to Long-tErm care Residents (SIMPLER): study protocol for a cluster randomised controlled trial. Trials; 19(1):37.

\section{P159 | First-in-human, randomized study to assess the safety, tolerability, immunogenicity, and shedding of a live- attenuated respiratory syncytial virus vaccine}

\author{
Johan van der Plas ${ }^{1}$; Pauline Verdijk ${ }^{2}$; Emilie van Brummelen ${ }^{1}$; \\ Meta Roestenberg ${ }^{3}$; Jacobus Burggraaf ${ }^{1}$; Ingrid Kamerling ${ }^{1}$ \\ ${ }^{1}$ Centre for Human Drug Research; ${ }^{2}$ Intravacc; ${ }^{3}$ Department of \\ Parasitology and Infectious Diseases, Leiden University Medical Center
}

Introduction: Respiratory syncytial virus (RSV) is a leading cause of hospitalization of children under 5 years of age. Currently, there is no effective treatment for an ongoing RSV infection nor is there a safe and effective vaccine available. Therefore, Intravacc developed a liveattenuated recombinant RSV vaccine. With reverse genetics, a virus was constructed lacking the $\mathrm{G}$ attachment protein (RSV $\Delta \mathrm{G}$ ) resulting in a live-attenuated virus that induced a potent immune response in pre-clinical studies. We performed a first-in-human, randomized, double-blind, placebo-controlled trial to assess the safety, tolerability, viral shedding, and immunogenicity of RSV $\Delta \mathrm{G}$ in healthy adults.

Methods: Healthy adult volunteers $(n=48)$ were randomized in a vaccine:placebo ratio of 3:1 to receive a single dose of $6.5 \pm 0.5 \mathrm{CCID}_{50}$ RSV $\Delta \mathrm{G}$ or placebo intranasally. Subjects were eligible for inclusion if they had virus neutralization antibody titres $(V N T) \leq 9.6 \log _{2}$. Safety was assessed by vital signs, routine blood chemistry and hematology assessment, nasal examination, and oral temperature. Tolerability was assessed by a visual analogue pain scale. Symptom scores were derived from solicited adverse events (nasal congestion, epistaxis, rhinorrhoea, sneezing, coughing, sore throat, dyspnoea, malaise, headache, myalgia/arthralgia, earache, and eye irritation) recorded by subjects daily on a mobile application. Viral shedding was assessed by quantitative culture and PCR. Immunogenicity was determined both in serum (VNT, Palivizumab Competing Antibodies) and in nasal wash (VNT, IgA).

Results: Intranasal administration of RSV $\Delta \mathrm{G}$ was well tolerated, and safety analysis did not show findings of clinical concern. All treatment emergent adverse events recovered without sequelae during the first 28 days (mild $[n=74]$, moderate $[n=3]$, severe $[n=0]$ ). The number of solicited adverse events (figure) and symptom scores were similar for the RSV $\Delta G(n=36)$ and placebo group $(n=12)$ (Figure 1$)$. No 
infectious virus was detected in nasal wash samples. No significant change in serum VNT was observed after vaccination compared to placebo. Analysis of the other immunogenicity parameters in serum and nasal wash did not show an evident immune response.

Conclusions: In conclusion, RSV $\Delta \mathrm{G}$ is safe and well tolerated, but the virus could be too attenuated or a dose of $6.5 \pm 0.5 \log _{10} \mathrm{CCID}_{50}$ could be too low to elicit an immune response in healthy adults. Preexisting immunity against RSV complicates the assessment of immunogenicity in healthy adults. Immunogenicity can ultimately be best assessed in the RSV-naïve population. Further research investigating the safety and immunogenicity of RSV $\Delta \mathrm{G}$ in seropositive and eventually seronegative infants appears to be warranted.

\section{P161 | Quantification of mycophenolic acid in human plasma by LC-TOF-MS for therapeutic drug monitoring}

Natalicia de Jesus Antunes ${ }^{1}$; Karin Kipper ${ }^{2}$;

Jason Alexander Raymond ${ }^{3}$; Nina L. Regina Ince ${ }^{3}$; Lewis Couchman ${ }^{4}$; Atholl Johnston ${ }^{5}$

${ }^{1}$ Queen Mary University of London; ${ }^{2}$ Analytical Services International (ASI), St George's-University of London, Cranmer Terrace, London, United Kingdom; and Institute of Chemistry, University of Tartu, Tartu, Estonia;

${ }^{3}$ Analytical Services International (ASI), St George's-University of London, Cranmer Terrace, London, United Kingdom; and Department of Chemistry, Faculty of Engineering and Physical Science, University of Surrey, Guildford, Surrey, United Kingdom; ${ }^{4}$ Analytical Services International (ASI), St George's-University of London, Cranmer Terrace, London, United Kingdom; and Pharmaceutical Sciences Clinical Academic Group, King's College London, London, United Kingdom; ${ }^{5}$ Analytical Services International (ASI), St George's-University of London, Cranmer Terrace, London, United Kingdom; and Clinical Pharmacology, William Harvey Research Institute, Barts and The London School of Medicine and Dentistry, Queen Mary University of London, London, United Kingdom

Introduction/Background \& Aims: Mycophenolic acid (MPA) is an immunosuppressant drug used for the prevention of organ transplant rejection, such as renal, liver, lung, or heart transplantation [1]. The aim of the study was to develop and validate, for the first time, a liquid chromatography and time-of-flight mass spectrometry (LC-TOFMS) detection to measure mycophenolic acid to quantify the drug in patient samples as part of the routine therapeutic drug monitoring (TDM) service.

Method/Summary of work: MPA was extracted from $50 \mu \mathrm{L}$ human plasma by protein precipitation, using sulindac as internal standard (IS). The separation of MPA was obtained on $150 \times 2.1 \mathrm{~mm}, 5 \mu \mathrm{m}$ Luna Omega polar C18 column (Phenomenex) kept on $40^{\circ} \mathrm{C}$. The mobile phase consisted of a mixture of acetonitrile/de-ionised water $(50 / 50, v / v)+0.1 \%$ formic acid at a flow rate of $350 \mu \mathrm{L} / \mathrm{min}$. Analyte and IS were monitored on an Agilent 6230 TOF-MS using a Jet-Stream (electrospray) interface running in positive mode. Assay performance was evaluated by analysing external (Proficiency Testing [PT]) samples. The method was used to evaluate the drug level in plasma of patients $(n=15)$ to therapeutic drug monitoring.

Results/Discussion: The retention time was $2.33 \mathrm{~min}$ and $2.31 \mathrm{~min}$ for MPA and sulindac, respectively, and no carry-over was observed. The lower limit of quantification (LLOQ) of MPA was $0.1 \mu \mathrm{g} / \mathrm{mL}$. The within and between assay reproducibility results were within the assay acceptance criteria ( $\leq 20 \%$ for LLOQ and $\leq 15 \%$ for quality controls). The results for matrix effects are considered acceptable. MPA demonstrated stability in plasma after 6 days at room temperature and at $4^{\circ} \mathrm{C}, 4$ days in the auto-sampler at $10^{\circ} \mathrm{C}, 5$ months at $-20^{\circ} \mathrm{C}$, and after 3 freeze/thaw cycles, with accuracy ranging from $91.56 \%$ to 100.62\%. The PT samples results are presented on Table 1 . The patients samples quantification was comparable to the previous method.

Conclusion(s): This method showed satisfactory analytical performances for the determination of MPA in plasma over the calibration range of $0.1 \mu \mathrm{g} / \mathrm{mL}$ to $15.0 \mu \mathrm{g} / \mathrm{mL}$.

\section{REFERENCE(S)}

1. Kaufman DB, Shapiro R, Lucey MR, et al. Immunosuppression: practice and trends. Am J Transplant 2004; 4 Suppl. 9: 38-53.

TABLE 1 Proficiency testing samples of mycophenolic acid

\begin{tabular}{|c|c|c|c|c|c|}
\hline \multirow{2}{*}{$\begin{array}{l}\text { PT } \\
\text { External report }\end{array}$} & \multicolumn{3}{|c|}{ Concentration $(\mu \mathrm{g} / \mathrm{mL})$} & \multirow{2}{*}{$\begin{array}{l}\text { Measured/external } \\
\text { ratio (\%) }\end{array}$} & \multirow{2}{*}{$\begin{array}{l}\text { Measured/previous } \\
\text { ratio (\%) }\end{array}$} \\
\hline & Previous method & Meas & & & \\
\hline 1 & 5.62 & 6.1 & 5.96 & 106.05 & 97.70 \\
\hline 2 & 1.39 & 1.4 & 1.51 & 108.63 & 107.86 \\
\hline 3 & 2.04 & 2.0 & 2.26 & 110.78 & 113.00 \\
\hline 4 & 2.03 & 2.0 & 2.38 & 117.24 & 119.00 \\
\hline 5 & 14.50 & 14.5 & 15.15 & 104.48 & 104.48 \\
\hline 6 & 4.30 & 4.3 & 4.76 & 110.70 & 110.70 \\
\hline
\end{tabular}




\section{P162 | Trends in oral anticoagulant prescribing in individuals with type 2 diabetes mellitus: A population-based study in the United Kingdom}

\author{
Hassan Alwafi ${ }^{1}$; Li Wei ${ }^{1}$; lan Wong ${ }^{2}$ \\ ${ }^{1}$ UCL; ${ }^{2}$ University of Hong Kong
}

Introduction/Background \& Aims: Diabetes mellitus and cardiovascular diseases often coexist. The aim of this study was to evaluate oral anticoagulant (OAC) prescribing trends in type 2 diabetes mellitus (T2DM) in the United Kingdom (UK) from 2001 to 2015.

Method/Summary of work: A cross-sectional study was conducted using electronic health records from The Health Improvement Network (THIN) primary care database of the UK. The prescribing trends of oral anticoagulant medications in individuals with T2DM were examined from 2001 to 2015, stratified by age, gender, and therapeutic classifications.

Results/Discussion: A total of 361,635 individuals with T2DM were identified, of which 36,570 were prescribed OACs from 2001 to 2015. The prevalence of OAC prescribing increased by $50.8 \%$ [from 4.4 (95\% confidence intervals, $\mathrm{Cl}, 4.2-4.6)$ in 2001 to $6.6(95 \% \mathrm{Cl}$ 6.5-6.7) in 2015 per 100 persons]. The prevalence of warfarin prescribing decreased by $13.9 \%$ [from $98.9(95 \% \mathrm{Cl} 98.4-99.4)$ in 2001 to 85.1 (95\% Cl 84.6-85.7) in 2015 per 100 persons]. This corresponded with increased prescribing of direct oral anticoagulants (DOACs) [from $0.1(95 \% \mathrm{Cl} 0.08-0.23)$ in 2010 to $17.6(95 \% \mathrm{Cl}$ 17.1-18.2) in 2015 per 100 persons] during the same period.

Conclusion(s): Prescribing of OACs in individuals with T2DM increased from 2001 to 2015. Since the introduction of DOACs, there has been a clear shift in prescribing towards these agents. Future studies are needed to assess the safety of the coadministration of oral anticoagulant medications and antidiabetic therapy with T2DM.

\section{P163 | An audit of polypharmacy on a general medical ward}

Savannah Haworth ${ }^{1}$; Simon Hill $^{2}$; Claire Rivett $^{2}$; Steve Wiltshire ${ }^{2}$; Alexander Dyker ${ }^{2}$

${ }^{1}$ Newcastle University; ${ }^{2}$ Newcastle Upon Tyne Hospitals NHS Foundation Trust

\section{Introduction/Background \& Aims:}

- Polypharmacy is an important and growing public health issue, in the UK and worldwide. Recent publications call for change in our management of patients taking multiple medications. Key priorities include identifying patients at risk of harm from polypharmacy and carrying out structured, patient-centered, medication reviews.

- This study did not require ethics approval

Aims:

- Standard: $100 \%$ of patients, over 65 , on 6 or more pre-admission medications (PAMs) should have a structured medication review, or the need for a review, documented in their hospital notes.

- The standard is based upon NICE Guidance 5: Medicines Optimisationsection $1.4 .1^{1}$ and RPS polypharmacy guidance ${ }^{2}$

- We would like to assess:

- How many patients meet this standard

- The scale of polypharmacy in medical inpatients

- The level of knowledge patients have about their medications

- If patients are prescribed any potentially inappropriate prescriptions (PIPs)

Method/Summary of work:

- Cross-sectional audit of patients on a GIM ward, RVI, on a single day.

- Inclusion criteria: over 65 , on 6 or more PAMs, able to answer questions.

- Data were collected for the current admission from patient notes, eRecord, and a patient interview.

- Patients were asked what medications they knew they took, and this was compared to their prescribed medications.

- PIPs were assessed using the STOPP/START criteria $^{3} \&$ review with a prescribing pharmacist and specialist registrar.

Results/Discussion:

- 21 of 29 patients (72.4\%) met inclusion criteria.

- 0 patients had a structured polypharmacy medication review, or the need for one, documented.

- $57 \%$ of patients were prescribed $\geq 1$ PIPs.

- The most common PIP was omeprazole $(n=4)$.

Conclusion(s):

- Polypharmacy is not recognised or addressed routinely in medical inpatients.

- Patients are not receiving, or being flagged as needing, medication reviews for polypharmacy.

- Many patients had potentially inappropriate prescriptions.

- Recommendations:

- Ensure patients on polypharmacy have structured and documented medication reviews.

- Introduction of a system to identify those who have/haven't been reviewed.

- Re-audit post intervention. 
TABLE 1

\begin{tabular}{|llll}
\hline Age group & $\begin{array}{l}\text { Mean number of } \\
\text { medications (range) }\end{array}$ & $\begin{array}{l}\text { Mean percentage of } \\
\text { medications known }\end{array}$ & $\begin{array}{l}\text { Percentage of patients who feel } \\
\text { they're on too many medications }\end{array}$ \\
\hline All patients $(n=21)$ & $12.4(6-24)$ & $55 \%$ & $48 \%$ \\
like to stop some of their medications
\end{tabular}

\section{REFERENCE(S)}

1. National Institute for Health and Care Excellence. Medicines optimisation: the safe and effective use of medicines to enable the best possible outcomes. NICE, 2015. https://www.nice.org.uk/guidance/ng5 (Accessed 10.01.2019)

2. The Royal Pharmaceutical Society. Polypharmacy: getting our medicines right. 2018. https://www.rpharms.com/recognition/ setting-professional-standards/polypharmacy-getting-our-medicinesright. (Accessed 18/02/19)

3. O'Mahoney D, et al. STOPP/START criteria for potentially inappropriate prescribing in older people: version 2. Age and Ageing. 2015;44(2): 213-218. https://doi.org/10.1093/ageing/afu145 (Accessed $19 / 02 / 19$

\section{P164 | Doctor shopping of oxycodone in France: First nationwide patterns by sex, age, formulation, and dosage}

\author{
Thomas Soeiro ${ }^{1}$; Vincent Pradel $^{2}$; Clémence Lacroix $^{2}$; \\ Maryse Lapeyre-Mestre ${ }^{3}$; Joëlle Micallef ${ }^{2}$ \\ ${ }^{1}$ Centre d'évaluation et d'information sur la pharmacodépendance - \\ Addictovigilance, Assistance Publique - Hôpitaux de Marseille; ${ }^{2}$ Centre \\ d'Évaluation et d'Information sur la Pharmacodépendance - \\ Addictovigilance, Service de Pharmacologie clinique, Centre hospitalo- \\ universitaire de Marseille, France; ${ }^{3}$ Centre d'Évaluation et d'Information \\ sur la Pharmacodépendance - Addictovigilance, Service de \\ Pharmacologie clinique, Centre hospitalo-universitaire de Toulouse, \\ France
}

Introduction/Background \& Aims: Determinants of abuse include pharmacological parameters such as pharmacodynamics, pharmacokinetics, and dosage [1] and characteristics of subjects such as age, and gender [2]. To our knowledge, there is no study that specifically assessed the role of age, sex, and formulation on oxycodone abuse. Therefore, in the international context of oxycodone overuse, subsequent abuse, and related deaths, the study assessed doctor shopping of oxycodone by sex, age, formulation, and dosage, in the whole French population, using a method accounting for overlapping prescriptions.

Method/Summary of work: We previously validated a method accounting for overlapping prescriptions to quantify doctor shopping from the databases of the French Health Insurance $[1,3]$. The method demonstrated its relevance in noticeably identifying doctor shopping of buprenorphine, methylphenidate, and benzodiazepines [1,3]. All dispensing of any oxycodone-containing medication to the whole French population in 2016 was extracted from the databases of the French Health Insurance, which covers the 66.6 million inhabitants in France. The doctor-shopping indicator (DSI) is a proxy for abuse defined as the proportion of medication obtained by overlapping prescriptions. According to previous studies in several geographical areas and for several classes of medications, a DSI higher than the threshold of $1 \%$ is a signal of abuse [1,3].

Results/Discussion: In the whole French population in 2016, $1115.5 \mathrm{~kg}$ of oxycodone, corresponding to $1673.3 \mathrm{~kg}$ of morphine equivalent, was dispensed to 212757 subjects. Stratified by sex and age, the DSI was $9.6 \%$ for the 18- to 24 -year-old women, $2.3 \%$ for the 25 - to 34 -year-old women, and $1.5 \%$ for the 35 - to 44 -year-old women. The DSI was $3.2 \%$ for the 25 - to 34 -year-old men and $2.3 \%$ for the 35- to 44 year-old men. Stratified by formulation and dosage, the DSI was $1.2 \%$ for the $20 \mathrm{mg}$ immediate-release tablets and $1.8 \%$ for the $120 \mathrm{mg}$ extended-release tablets (Figure 1).

Conclusion(s): Results showed evidence of doctor shopping for the 18- to 44-year-old subjects with a maximum for the 18- to 24-yearold women, and for the highest dosages for both immediate-release and extended-release tablets. Patterns of doctor shopping by sex, age, formulation, and dosage provide useful data to health professionals and regulatory authorities to improve the security of prescriptions of oxycodone and may contribute to awareness of physician to avoid trivializing oxycodone.

\section{REFERENCE(S)}

1. Pradel V, Delga C, Rouby F, Micallef J, Lapeyre-Mestre M. Assessment of abuse potential of benzodiazepines from a prescription database using 'doctor shopping' as an indicator: CNS drugs. 2010 Jul;24(7): 611-20.

2. Becker JB, McClellan ML, Reed BG. Sex differences, gender and addiction: sex, gender, and addiction. Journal of Neuroscience Research. 2017 Jan 2;95(1-2):136-47.

3. Ponté C, Lepelley $M$, Boucherie Q, Mallaret $M$, Lapeyre Mestre $M$, Pradel V, et al. Doctor shopping of opioid analgesics relative to benzodiazepines: a pharmacoepidemiological study among 11.7 million inhabitants in the French countries. Drug and Alcohol Dependence. 2018 Jun;187:88-94. 

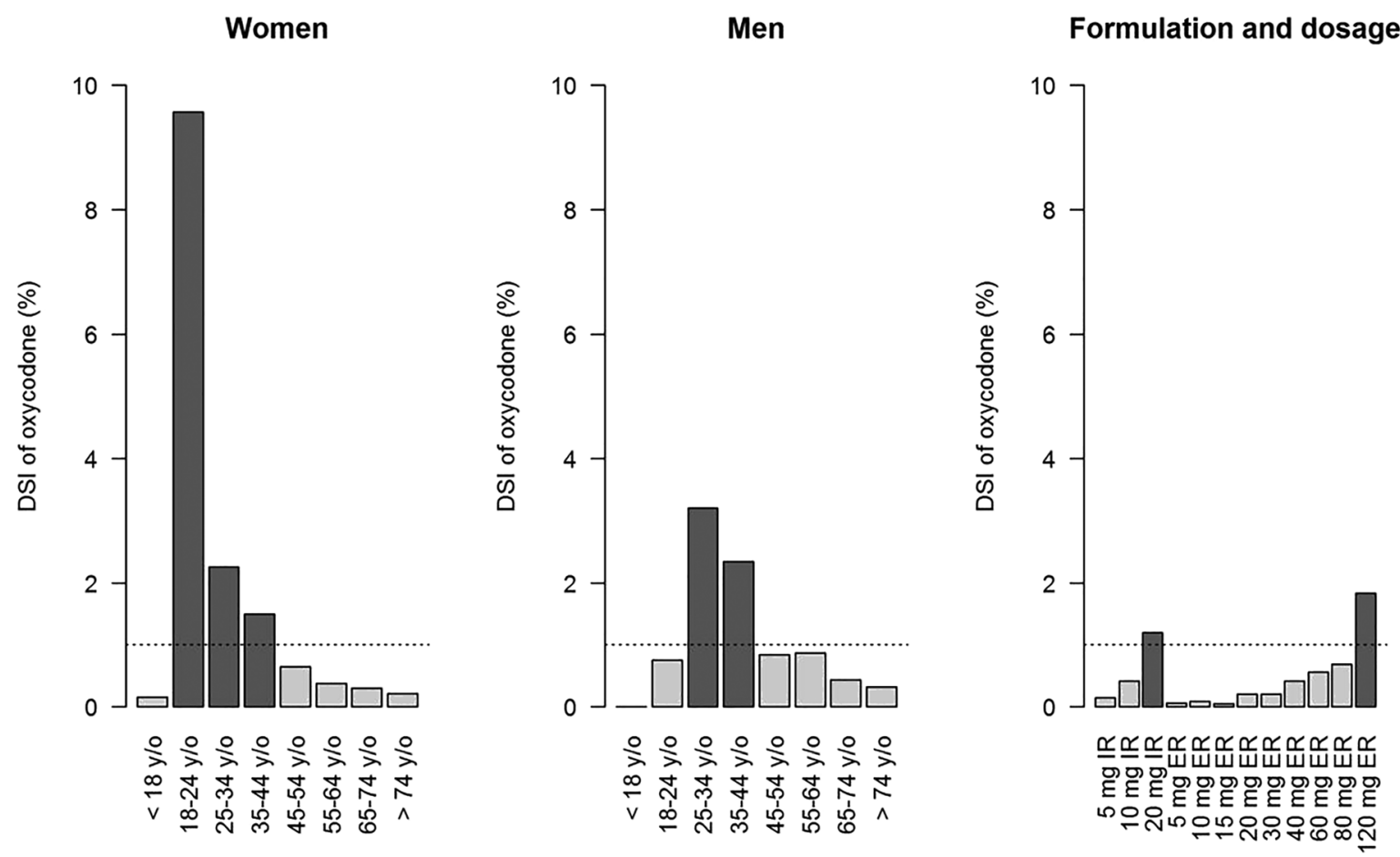

FIGURE 1 DSI of oxycodone by sex, age, formulation, and dosage in the whole French population in 2016. DSI: doctor shopping indicator; IR: immediate-release; ER: extended-release

\section{P165 | Perspectives of deprescribing held by New Zealand nurse and pharmacy professionals}

\author{
Denise Taylor ${ }^{1}$; Betty Poot $^{1}$; Leonie Walker ${ }^{1}$; Jill Wilkinson ${ }^{2}$; \\ Gigi Lim ${ }^{3}$; Rebecca Zonneveld ${ }^{1}$ \\ ${ }^{1}$ Victoria Univesrity of Wellington; ${ }^{2}$ New Zealand Nursing; ${ }^{3}$ University of \\ Auckland
}

Introduction/Background \& Aims: Deprescribing involves the critical review for the need of multiple medicines taken by an individual. This review is based on clinical and individual need for a medicine as well as consideration of best practice in any disease area. The need for deprescribing is viewed to be greatest for older people who are prescribed medicines more frequently and often by multiple prescribers. The presence of co-morbidity, complex clinical situations, and multiple prescribers increases reluctance of others to stop a medication. The only published research on New Zealand (NZ) clinical practitioner's perspectives of deprescribing involved general practitioners and hospital clinicians who expressed concern about the possibility of doing more harm than good by stopping long-term medicines. ${ }^{1,2}$ They also shared that effective deprescribing may require the expertise of other members of the multidisciplinary team. Importantly, nurses, nurse practitioners, pharmacist prescribers, and pharmacists also have a role in this area. ${ }^{1,2}$ Our study aimed to explore the perspectives and knowledge of pharmacy and nurse professionals on deprescribing.
Method/Summary of work: A qualitative design using focus groups and interviews was utilised. Our study recruited 18 pharmacists and 17 nurses who participated in one of 6 focus groups or 7 interviews in the following cohort groups: nurse practitioners (independent prescribers $n=7)$; registered nurse prescribers $(n=6)$; non-prescribing nurses $(n=4)$; pharmacist prescribers $(n=6)$; hospital pharmacist nonprescribers $(n=8)$; and community pharmacists $(n=4)$. We digitally recorded each session translated verbatim and analysed using an iterative thematic analysis approach, including examining within and across professional groups. Victoria University of Wellington research ethics approval 0000026829 granted 30-11-2018.

Results/Discussion: Findings reveal variation in knowledge, perspectives, understanding, and/or involvement in deprescribing by individual prescribers and by professional groups. Nurse practitioners had more experience and greater confidence in deprescribing, than registered nurse prescribers who only felt competent to change medications they were familiar with and/or prescribed. Pharmacists expressed greater confidence in reviewing and deprescribing all medicines, and this was directly linked with their perceived expertise and familiarity with the broad range of medicines they worked with daily. There was intra and inter cohort differences in opinion on who was or was not appropriately trained to be involved in deprescribing, seemingly linked to role protection.

Conclusion(s): The findings highlight an important area for nurse and pharmacist education and professional development in the areas of medicines review and deprescribing. Further research is required on developing and supporting collaborative approaches to deprescribing within the multidisciplinary team. 


\section{REFERENCE(S)}

1. Allabouni, N.J., Nichtala, P.S., Mangin D., Tordoff J.M. (2016) Challenges and enablers of deprescribing: a general practitioner perspective. PLOS ONE|DOI:https://doi.org/10.1371/journal.pone.0151066.

2. Jamieson, H. A., Nishtala, P. S., Scrase, R., Deely, J. M., Abey-Nesbit, R., Connolly, M. J., et al. (2018). Drug burden and its association with falls among older adults in New Zealand: a national population crosssectional study. Drugs \& Aging, 35(1), 73-81.

\section{P166 | Pregnancy protection and pregnancies in women prescribed angiotensin-converting enzyme inhibitors/angiotensin receptor blockers}

\author{
Elizabeth Lovegrove $^{1}$; John Robson ${ }^{2}$; Patricia McGettigan ${ }^{3}$ \\ ${ }^{1}$ Barts Health NHS Trust; ${ }^{2}$ Clinical Effectiveness Group, Queen Mary \\ University of London; ${ }^{3}$ William Harvey Research Institute, Barts and the \\ London School of Medicine and Dentistry
}

Introduction/Background \& Aims: The teratogenic risks of angiotensin-converting enzyme inhibitors (ACEi) and angiotensin receptor blockers (ARB) are well documented. Prescribing of these in younger women in primary care is frequent and increasing. The aim of this study was to assess how frequently women of childbearing age, who are prescribed ACEi/ARBs, receive pre-conception advice, contraception advice and/or are prescribed contraception and to record the number of pregnancies in this population.

Method/Summary of work: This was a cross-sectional study conducted among patients from 141 general practices in East London. Women aged 15-45 with an ACEi/ARB prescription issued between $1 / 10 / 18$ and $1 / 1 / 19$ were included. Women with a previous hysterectomy or sterilisation were excluded. An electronic search strategy was designed to extract pseudonymised data concerning preconception and contraception advice, contraception and pregnancies from the electronic clinical system and applied to the selected cohort on 1/1/19. Data were analysed in 5-year age groups using univariate descriptive statistics.

Results/Discussion: Of 302,939 women aged 15-45, 2651 (0.9\%) were prescribed an ACEi/ARB in a 3-month period. Of these 2651 $1.32 \%$ had pre-conception advice recorded; $8.68 \%$ had contraception advice recorded; $81.44 \%$ had no advice and no contraception recorded; 100 pregnancies and 21 terminations/ miscarriages/abortions were recorded over 12 months. No women aged 15-19 and only $11.8 \%$ of women aged 20-24 had prepregnancy advice or contraception recorded. Recording of advice and contraception also decreased for women above 35, who formed a larger "at-risk" group because of more frequent ACEi/ARB prescriptions.

Conclusion(s): Our study has found that recording of pre-pregnancy advice and contraception in women prescribed ACEi/ARBs is suboptimal and rates of recording also varies with age. This may place women and their babies at risk of exposure to these teratogens during pregnancy, and our study shows an important number of pregnancies do occur in this population. Our findings indicate the need for improved primary care-based safety strategies such as patient held records, signed consent for understanding risks in pregnancy, virtual review systems, and clinician education.

\section{P167 | Identification of a complex of symptoms frequently caused by commonly prescribed drugs as a potential tool to quantify the benefits of deprescribing}

Oliver Mercer ${ }^{1}$; Christopher Threapleton ${ }^{2}$; Fiona Bennett ${ }^{3}$; Emma Baker ${ }^{2}$

${ }^{1}$ St George's, University of London/Tameside \& Glossop IC NHS FT; ${ }^{2}$ St George's, University of London; ${ }^{3}$ University College London Hospitals NHS Foundation Trust

Introduction/Background \& Aims: The prevalence of polypharmacy and its potential harms are well documented. By contrast, the benefits of deprescribing are more difficult to quantify. An important aim of deprescribing is to reduce symptomatic adverse drug reactions. However, in patients with multimorbidity, it can be difficult to tell whether a patient's symptoms are due to their diseases, their medications, or a combination.

The aim of our study was to identify the symptoms commonly associated with the 100 drugs and classes most commonly prescribed in the UK as a prelude to developing a tool to quantify the benefits of deprescribing.

Method/Summary of work: We have previously described the 100 drugs and classes most commonly prescribed in the UK and, within classes, the most commonly prescribed individual drugs. ${ }^{1}$ For each drug on this list, the summary of product characteristics (SmPC) was used to identify all very common $(\geq 1 / 10)$ and common $(\geq 1 / 100$, $<1 / 10$ ) undesirable effects. To ensure all common undesirable effects were captured, this was repeated using a different SmPC for each drug where possible using a different dose or manufacturer. A list of undesirable effects was generated, and frequency of occurrence was described as the number of drugs/classes from the top 100 causing each effect.

Results/Discussion: A total of 396 different very common or common undesirable effects caused by the 100 drug classes were identified. Table 1 shows all undesirable effects caused by more than $10 \%$ of drugs, along with the number of drugs/classes causing that effect. For example, $53 \%$ of drugs on the list commonly cause nausea, and $47 \%$ commonly cause headache.

Conclusion(s): Adverse drug reactions are an important but underrecognised cause of illness, particularly in patients with multimorbidity. Our study highlights a complex of symptoms commonly associated with commonly prescribed drugs. Further work is now required to establish whether this could form the basis of a tool to 
TABLE 1 Undesirable effects caused by more than $10 \%$ of the top 100 drugs

\begin{tabular}{|c|c|}
\hline Undesirable effect & $\begin{array}{l}\text { Number of drug classes } \\
\text { causing the undesirable } \\
\text { effect }\end{array}$ \\
\hline Nausea & 53 \\
\hline Headache & 47 \\
\hline Dizziness & 41 \\
\hline Diarrhoea & 40 \\
\hline Vomiting & 40 \\
\hline Rash & 30 \\
\hline Abdominal pain/discomfort & 29 \\
\hline Fatigue & 28 \\
\hline Dry mouth & 24 \\
\hline Somnolence/drowsiness & 23 \\
\hline Constipation & 21 \\
\hline Dyspepsia & 20 \\
\hline Itch/pruritis & 20 \\
\hline Asthenia & 16 \\
\hline Sleep disturbance & 16 \\
\hline Hypotension & 13 \\
\hline Paraesthesia & 12 \\
\hline $\begin{array}{l}\text { Reflex changes (increase, decrease } \\
\text { or absence) }\end{array}$ & 12 \\
\hline Dysgeusia (distorted sense of taste) & 12 \\
\hline Tremor & 11 \\
\hline Visual disturbance (including diplopia) & 11 \\
\hline Flatulence & 11 \\
\hline Confusion & 11 \\
\hline
\end{tabular}

identify patients requiring medication review and/or to quantify the benefits of deprescribing.

\section{REFERENCE(S)}

1. Audi S, Burrage DR, Lonsdale DO, et al. The 'top 100' drugs and classes in England: an updated 'starter formulary' for trainee prescribers. $\mathrm{Br} J$ Clin Pharmacol. 2018;84(11):2562-2571.

\section{P168 | Evaluation of pyridoxic acid as endogenous biomarker of OAT1/3 transporters using modelling and simulation approach}

Amais Ahmad ${ }^{1}$; Kayode Ogungbenro ${ }^{2}$; Jan Snoeys ${ }^{3}$;

Amin Rostami-Hodjegan ${ }^{2}$; Aleksandra Galetin ${ }^{2}$

${ }^{1}$ University of Manchester; ${ }^{2}$ Centre for Applied Pharmacokinetic

Research, University of Manchester; Certara UK Limited (Simcyp Division),

Sheffield; ${ }^{3}$ Janssen Pharmaceuticals

Introduction/Background \& Aims: Drug-drug interaction (DDI) risk is usually evaluated using in vitro-in vivo extrapolation approach, as supported by regulatory guidance documents (1). Although this approach has been well established for drug metabolizing enzymes, there are limitations in this approach to predict transporter related DDIs (2). The issue may be resolved by using endogenous biomarkers as early indicators of potential transporter-mediated DDIs (2). Recently, plasma pyridoxic acid (PDA) was proposed as a novel endogenous biomarker for the assessment of DDI risk via renal organic anion transporters (OAT)1/3. The study objective was to develop biomarker population model in NONMEM to support its qualification.

Method/Summary of work: A randomized crossover study was performed in 6 healthy fasted subjects. A mechanistic model (Figure 1A) was simultaneously fitted to plasma and urine PDA data in the presence and absence of OAT inhibitor (probenecid, $500 \mathrm{mg}$ qid). Independent literature-reported PDA dataset was used for model validation. Power calculations were performed for different dosing regimens (multiple or single dose) of hypothetical OAT inhibitors.

Results/Discussion: PDA renal clearance contributes $>85 \%$ to its total clearance. There is no effect of circadian rhythm on PDA baseline levels. The PDA model successfully described biomarker baseline and OAT-mediated interaction data. Comparable sample sizes were required for dosing regimens of strong and medium OAT inhibitors of the biomarker for nominal power level.

Conclusion(s): Model-based simulations and power calculations confirmed sensitivity of PDA to identify moderate and weak OAT inhibitors in an adequately powered clinical study and can support design of prospective OAT interaction studies.

\section{REFERENCE(S)}

1. 2017 FDA Drug-drug interaction studies guidance for industry, https://www.fda.gov/downloads/Drugs/Guidances/UCM581965.pdf.

2. Guo, Yingying et al. "Advancing predictions of tissue and intracellular drug concentrations using in vitro, imaging and physiologically based pharmacokinetic modeling approaches." Clinical Pharmacology and Therapeutics 5 (2018): 865-889. 
(A)

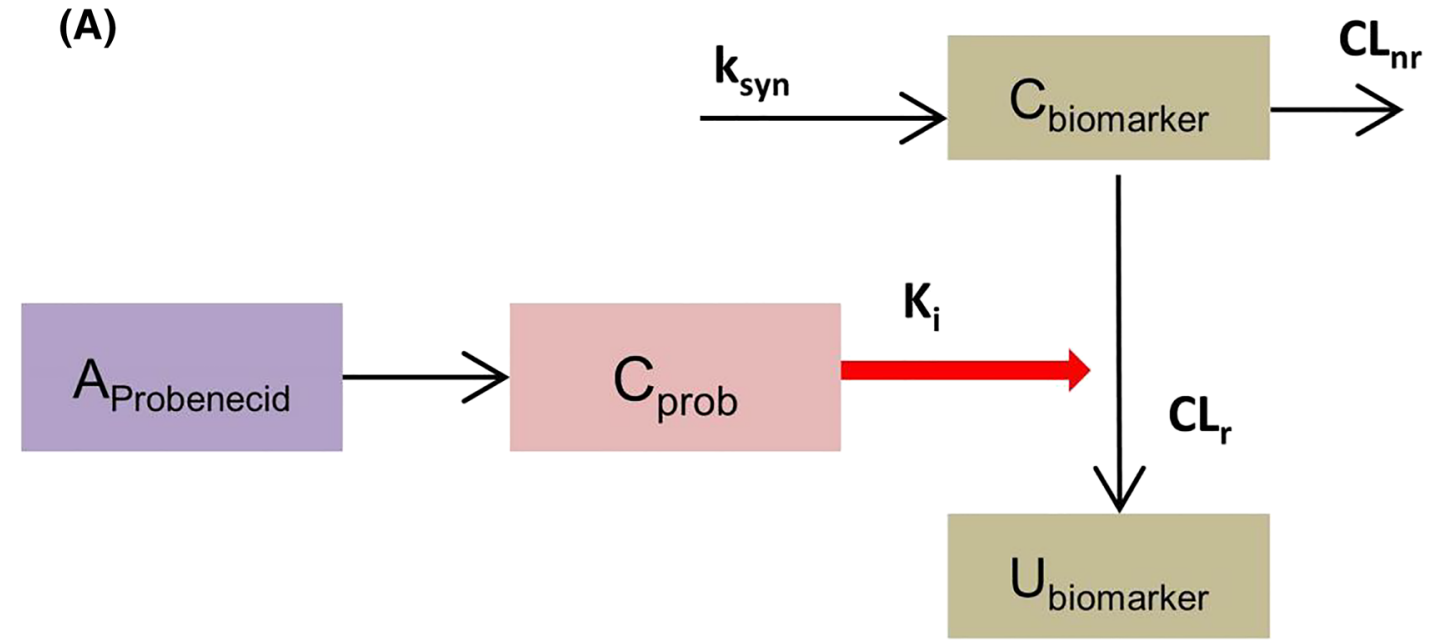

(B)
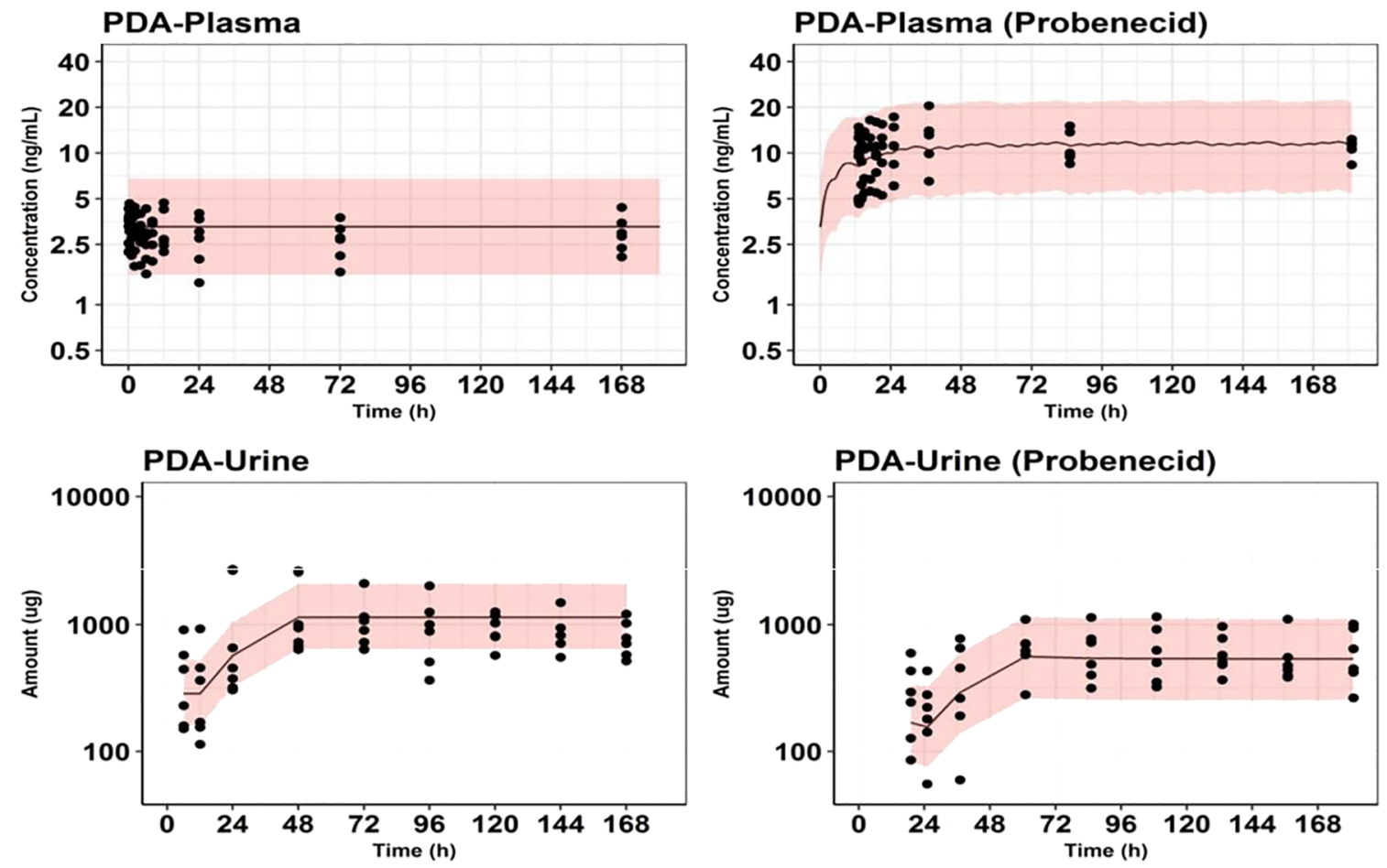

FIGURE 1 (A) Structure of the mechanistic PK model for PDA as OAT endogenous biomarker. (B) Visual predictive checks of the developed population PK model for PDA plasma and urine data, superimposed with the observed data. The shaded area represents the $90 \%$ prediction of the simulated data, and the black dots are the observed data.

\section{P169 | Impact of bariatric surgery on opioids consumption: A pharmaco-epidemiological study in the French health insurance database}

Cécile Conte $^{1}$; Patrick Ritz ${ }^{2}$; Maryse Lapeyre-Mestre ${ }^{1}$

${ }^{1}$ Clinical Pharmacology Department, Clinical Investigation Center (CIC 1436) Toulouse University Hospital, Inserm unit 1027, University of

Toulouse $3 ;{ }^{2}$ Inserm unit 1027 , University of Toulouse 3 , Nutrition
Department, Toulouse CIO (Information and Orientation Center) and $\mathrm{CHU}$

Introduction/Background \& Aims: Obesity is associated with comorbid conditions including osteoarthritis and chronic pain. However, bariatric surgery's impact on analgesic drug consumption remains unknown in France. The main objective of this study was to describe variations in opioids consumption after bariatric surgery and to identify factors associated with a decreased used of these drugs 
Method/Summary of work: We conducted a pharmacoepidemiological retrospective cohort study from the French national health insurance database in the Midi-Pyrénées region between 2014 and 2016. All obese patients undergoing bariatric surgery were identified using hospital diagnosis and medical acts. The prevalence of opioid drugs consumption and the total defined daily dose (DDD) was calculated and compared between the year before surgery and the postoperative period (2 to 14 months following surgery).

Results/Discussion: Over the study period, 5362 patients underwent bariatric surgery. Before surgery, prevalence of mild opioids and stronger opioids was respectively $35.3 \%(n=1891)$ and $2.3 \%$ ( $n=124$ ). During the postoperative period, prevalence of mild opioids and stronger opioids decreased respectively at $23.5 \%(n=1263)$ and $1.7 \%(n=94)$ with statistical significance. Tramadol was the most prescribed both in the preoperative period ( $80 \%$ of patients) and in the postoperative period (70\% of patients). Morphine and oxycodone were the most prescribed (at least once prescription for $50 \%$ of patients in the preoperative period and for $40 \%$ of patients in the postoperative period). After surgery, $29.8 \%(n=1599)$ patients had decreased patterns of consumption, and 15.6\% ( $n=836)$ had increased patterns of consumption. Factors significantly associated with a decreased use of opioids were older age, absence of psychotropic drug use, and absence of comorbidities such as diabetes, osteoarthritis, and cancer.

Conclusion(s): These results revealed significant consumption of opioids in obese patients and a slight decrease in opioids consumption after bariatric surgery. Further analyses will be conducted to assess factors associated with variations in the postoperative period.

\section{REFERENCE(S)}

1. Narouze S, Souzdalnitski D. Obesity and chronic pain: systematic review of prevalence and implications for pain practice. Reg Anesth Pain Med. 2015 Mar-Apr;40(2):91-111.

\section{P170 | Pattern of drug information sources utilized by medical practitioners at a teaching hospital in Nepal}

\author{
${\text { Pravin } \text { Prasad }^{1} \text {; Naresh Karki }}^{2}$; Kamal Kandel ${ }^{2}$ \\ ${ }^{1}$ Maharajgunj Medical Campus; ${ }^{2}$ Lumbini Medical College and Teaching \\ Hospital
}

Introduction/Background \& Aims: Development of pharmaceutical industry has been enriching our knowledge about drugs continuously. It is not practical to remember each information.[1] Physicians need to be supplemented with new information using various drug information (DI) sources and should remain unbiased and reliable. Availability of unbiased and reliable sources of DI will help to enhance patient outcome and decrease the incidence of adverse drug reactions (ADR). [2] This study aims to understand the commonly used sources of DI
TAB LE 1 Types of drug queries faced by prescribers on day to day basis $(n=147)$

\begin{tabular}{|c|c|c|}
\hline Types of drug queries & Frequency & Percentage \\
\hline Dosage/administration & 136 & 92.50 \\
\hline Adverse drug reaction & 121 & 82.50 \\
\hline Contraindications & 119 & 81.25 \\
\hline Interactions & 108 & 73.75 \\
\hline Indications & 107 & 72.50 \\
\hline Cost/availability & 81 & 55.10 \\
\hline Efficacy & 79 & 53.75 \\
\hline Drug therapy & 74 & 50.34 \\
\hline Poisoning & 62 & 42.50 \\
\hline Pharmacokinetics & 42 & 28.75 \\
\hline Pharmacodynamics & 40 & 27.50 \\
\hline Others & 17 & 11.25 \\
\hline
\end{tabular}

by prescribers at our institute, their usefulness, and the need of independent drug information unit at the institute.

Method/Summary of work: A cross-sectional descriptive study that included all bonafide prescribers of this institute and actively involved in patient care was conducted. Consenting participants were requested to fill in the study proforma sheet. Study proforma sheet was developed and pretested that can be divided into three sections: general information of the participant, present practice of seeking drug information, and opinion about necessity of independent drug information unit at the institute. All the data thus collected were entered using EpiData version 3.1 and were analyzed using SPSS version 16.

Results/Discussion: Filled in questionnaires were obtained from 147 prescribers (response rate $75.38 \%$ ) of which 101 (68.71\%) were interns. Almost all of the participants $(137,95.80 \%)$ used textbooks and online medical sites as a source of DI. Out of 137 participants using online medical sites as source of DI, 80 (58.39\%) classified them as sometimes biased. It was seen that only 33 (24.09\%) participants using textbooks as source of DI found their queries answered at all instances. The study also found out that 61 (41.25\%) participants believed that they will absolutely be benefitted by having an unbiased DI services at the hospital. Participants had multiple types of drug query on a day-to-day basis as shown in the table.

Conclusion(s): Though prescribers are surrounded by multiple DI sources, there is always some biasness associated with them. The information contained in these sources could be anywhere between insufficient to extensive which could lead prescribers not have their query solved. The need of independent DI services exists to cater the different queries prescribers have so that the patient care can be enhanced.

\section{REFERENCE(S}

1. George B, Rao PG. Assessment and evaluation of drug information services provided in a South Indian teaching hospital. Indian journal of pharmacology. 2005;37(5):315. 
2. Ali AA, Yusoff SM, Joffry SM, Wahab MSA. Drug information service awareness program and its impact on characteristics of inquiries at DIS unit in Malaysian public hospital. Archives of Pharmacy Practice. 2013;4 (1):9.

\section{P171 | Effect of lobun probiotics in patients with chronic kidney disease stage III and IV, placebo-controlled open-labeled study}

Priya Lakshmi ${ }^{1}$; Shanthi Babu ${ }^{2}$; M.S. Ahil ${ }^{2}$; M. Raadhika ${ }^{2}$; M. Susila ${ }^{2}$; M. Geetha ${ }^{2}$

${ }^{1}$ Institute of pharmacology; ${ }^{2} \mathrm{Mmc}$

Introduction/Background \& Aims: Chronic kidney disease is more common in Indian Population today. Although medication or renal replacement therapies delay the progression of CKD to some extent, more than 2 million people worldwide are diagnosed with end stage renal disease, which is a substantial burden for global health and economics. Retarding the progression of CKD and preventing the complication is a challenge for Nephrologists. Here, we extend our data on lobun probiotics which delays the progression of CKD ${ }^{1}$.

Aim: To study the clinical and biochemical effects of lobun probiotics in patients with stage III and IV CKD.

Method/Summary of work: This is an open-labelled, single-centred, prospective, placebo-controlled interventional trial to study the effect of lobun probiotics in stage III and stage IV chronic kidney disease patients attending the Department of Nephrology in Govt Rajaji hospital, Madurai, Tamil Nadu, India.

A total of 80 patients were chosen assigned into two groups each group comprising 40 patients each.

Group1: Probiotics twice daily morning and afternoon.

Group 2: B complex capsules twice daily morning and afternoon.

Treatment is continued for 12 weeks. RFT monitored periodically. Inflammatory markers like C-Reactive protein status is assessed. Patient quality of life is assessed by SF-36 score at the end of 3 months. The results were analysed between two groups using student paired $t$ test.
Results/Discussion: The results indicate that probiotics administration is safe and delays the progression of CKD as evidenced by the significant decrease in blood urea, serum creatinine, and degree of albuminuria in the probiotics-treated group compared with placebo-treated group. By preventing the development of key damaging uremic toxins and by targeting the process of bacterial protein fermentation in the gut. The concentrations of uremic toxins (urea, uric acid, and creatinine) were reduced when study subjects were treated with the probiotics at 90 billion CFU/day dosage at the end of 8 weeks and 12 weeks. There was also a statistically significant improvement in serum calcium and serum sodium levels. According to Salih AM., Bifidobacterium longum utilizes various phenolic and indole metabolic toxic compounds in colonic region as its nutrient for growth, these bacteria cleanse the blood stream of urea, uric acid, and creatinine. ${ }^{2}$

According to Palmer SC et al (2013), QOL in CKD patients is often compromised with levels of depression associated with a decrease in kidney function. Depression is associated with a substantially increased risk of death in people with CKD. ${ }^{3}$ The bidirectional signaling between the gastrointestinal tract and the brain, mainly through the vagus nerve, the so called "microbiota-gut-vagus-brain axis," is vital for maintaining homeostasis, and it may be also involved in the etiology of several metabolic and mental dysfunctions/disorders. Evidence substantiating the microbiota-gut-brain axis links alterations in gut microbiota with depression the effects of intestinal microbiota composition on brain function predominantly involve animal models of behavioral disorders such as anxiety, depression, and cognitive dysfunction; however, accumulating evidence suggests that the composition of the gut microbiota may also have a role in several other metabolic conditions that involve the CNS.

Conclusion(s): From this study, it is evidenced that probiotics decreases the progression in stage III and stage IV CKD patients.

\section{REFERENCE(S)}

1. Ranganathan N, Ranganathan P, Friedman EA, Joseph A, Delano B, Goldfarb DS, Tam P, Rao AV, Anteyi E, Musso CG. Pilot study of probiotic dietary supplementation for promoting healthy kidney function in patients with chronic kidney disease. Advances in therapy. 2010 Sep 1; 27(9):634-47

\section{TABLE 1}

\begin{tabular}{|c|c|c|c|c|}
\hline \multirow[t]{2}{*}{ Groups } & \multicolumn{2}{|c|}{ Probiotics $(n=40)$} & \multicolumn{2}{|c|}{ Placebo $(n=40)$} \\
\hline & Baseline & 12th week & Baseline & 12th week \\
\hline Blood urea & $49.45 \pm 6.34$ & $44.7 \pm 7.56^{* * *}$ & $50.47 \pm 6.48$ & $52.75 \pm 8.08$ \\
\hline Serum creatinine & $2.94 \pm 0.88$ & $2.54 \pm 0.84^{* * *}$ & $2.75 \pm 0.68$ & $3.18 \pm 0.89$ \\
\hline Serum uric acid & $5.94 \pm 2.09$ & $5.49 \pm 1.72$ & $6.08 \pm 1.3$ & $6.31 \pm 1.23$ \\
\hline Serum calcium & $7.98 \pm 0.94$ & $8.89 \pm 0.95^{* * *}$ & $7.84 \pm 0.95$ & $8.22 \pm 0.76$ \\
\hline Serum phosphorus & $5.78 \pm 1.1$ & $5.43 \pm 1.05$ & $6.22 \pm 0.83$ & $6.38 \pm 0.67$ \\
\hline Serum sodium & $130.93 \pm 2.92$ & $132.8 \pm 3.02^{* *}$ & $132.1 \pm 3.02$ & $132.6 \pm 3.43$ \\
\hline Serum bicarbonate & $20.65 \pm 2.39$ & $22.75 \pm 2.04^{* *}$ & $21.15 \pm 1.96$ & $21.8 \pm 2.1$ \\
\hline Blood Hb\% & $9.09 \pm 0.53$ & $9.29 \pm 0.52^{*}$ & $9.05 \pm 0.63$ & $8.99 \pm 0.57$ \\
\hline SF-36 & $30.63 \pm 5.06$ & $33.5 \pm 4.12$ & $30 \pm 5.49$ & $28.5 \pm 5.1$ \\
\hline
\end{tabular}


2. Awn A, Sharif D, A Ahmed Al-Qais R. The beneficial effect of renadyl (kibow) probiotics on patients with chronic kidney diseases, with comparison between diabetic and nondiabetic patients, International Journal of Advances in Science Engineering and Technology, ISSN 2016: 2321.

3. Palmer SC, Vecchio M, Craig JC, Tonelli M, Johnson DW, Nicolucci A, Pellegrini F, Saglimbene V, Logroscino G, Hedayati SS, Strippoli GF. Association between depression and death in people with CKD: a meta-analysis of cohort studies. American Journal of Kidney Diseases. 2013 Sep 1;62(3):493-505

\section{P172 | Factors associated with the prescribing of high-dose opioids in primary care: A systematic review and meta-analysis}

\author{
Georgia Richards; Kamal Mahtani; Jeffrey Aronson; Tonny Muthee; \\ Nicholas DeVito; Carl Heneghan \\ University of Oxford
}

Introduction/Background \& Aims: The risks of harms from opioids increase substantially at high doses, and high-dose prescribing has increased in primary care. However, little is known about what leads to high-dose prescribing, and studies exploring this have not been synthesised. We, therefore, aimed to systematically synthesise factors associated with the prescribing of high-dose opioids in primary care.

Method/Summary of work: We searched Medline, Embase, Web of Science, reference lists, forward citations, and conference proceedings from database inception to 5 April 2019. We included observational studies with patient-level data in primary care settings of high-income countries that explored any factor(s) in people for whom opioids were prescribed, stratified by oral morphine equivalents (OME) in milligrams per day ( $\mathrm{mg} /$ day) where a group of participants received high doses ( $\geq 90$ OME mg/day). Two investigators independently screened studies, extracted data, and appraised the quality of included studies using the National Heart, Lung, and Blood Institute (NHLBI) Quality Assessment Tool for Observational Cohort and Cross-Sectional Studies. We pooled data on factors using random-effects meta-analyses and reported relative risks (RR) or mean differences with $95 \%$ confidence intervals (CI) where appropriate. We also performed a number needed to harm calculation on factors where applicable.
Results/Discussion: We included five studies with 4,056,714 participants taking opioids, of whom $3.75 \%$ ( $n=151,971$ ) were taking high doses. Included studies were graded as having fair to good quality evidence. The co-prescription of benzodiazepines, depression, male gender, and unemployment were significantly associated with the prescribing of high-dose opioids in primary care (Table 1).

Conclusion(s): Participants taking opioids in high doses are at greater risk of harm. Associated factors identify priority areas that can be taken into account when selecting a person for opioid therapy, identifying people who may be experiencing harm, and when managing people and services for people taking high-dose opioids in primary care.

\section{P173 | Off-label drug use practice of physicians other than pediatrists for $<12$-year-old children}

N. Ipek Kirmizi ${ }^{1}$; Volkan Aydin ${ }^{1}$; Narin Akici ${ }^{2}$; Banu Bayar ${ }^{3}$; Ahmet Akici ${ }^{1}$

${ }^{1}$ Marmara University; ${ }^{2}$ Haydarpasa Numune Training and Research Hospital; ${ }^{3}$ Turkish Medicines and Medical Devices Agency, Ministry of Health

Introduction/Background \& Aims: Off-label drug use (OLDU) is a special practice of pharmacotherapy that imposes additional responsibilities on the physician $[1,2]$. The conditions might be more compelling in certain patient groups that requires special attention, e.g. when it is needed for pediatric population managed by physicians other than pediatrists (PoPs). In this study, we aimed to examine the details of OLDU applications in younger children by PoPs.

Method/Summary of work: We collected data on all OLDU applications ( $\mathrm{n}=313$ ) submitted to the Turkish Medicines and Medical Devices Agency by PoPs in 2015 for <12-year-old children in this cross-sectional study. The age of patients, branches of physicians, and details on frequently used drugs and diagnoses were analyzed.

Results/Discussion: The mean age of the children for whom OLDU application was encountered was $7.5 \pm 3.7$ years. Among these, there

TAB LE 1 Meta-analyses of factors reported by included studies

\begin{tabular}{|c|c|c|c|c|c|c|}
\hline Factors & Outcomes RR (95\% Cl) & $12(\%)$ & No. of studies & $\begin{array}{l}\text { Sensitivity analyses } \\
\text { Outcomes RR (95\% CI) }\end{array}$ & $12(\%)$ & No. of studies \\
\hline 1. Coprescription of benzodiazepines & $2.27(1.03,5.02)^{*}$ & 99.7 & 3 & $1.47(1.37,1.59)^{*}$ & 0 & 2 \\
\hline 2. Depression & $1.38(1.27,1.51)^{*}$ & 0 & 2 & & & \\
\hline 3. Male gender & $1.21(1.12,1.30)^{*}$ & 56 & 5 & & & \\
\hline 4. Unemployment & $1.44(1.27,1.63)^{*}$ & 0 & 2 & & & \\
\hline 5. Age (mean difference) & 1.94 years $(-4.93,1.04)$ & 94 & 4 & -0.86 years $(-2.32,0.61)$ & 63.9 & 3 \\
\hline 6. Caucasian ethnicity & $1.09(0.98,1.20)$ & 20 & 2 & & & \\
\hline 7. Anxiety & $1.44(0.87,2.38)$ & 91 & 2 & & & \\
\hline
\end{tabular}

* Significantly associated with high-dose opioids; RR: relative risks; Cl: confidence intervals; $\mathrm{I}^{2}$ : a test of heterogeneity. If $\mathrm{I}^{2}>75 \%$ sensitivity tests were conducted. 
was no newborn group (<28 days); $31.3 \%$ of the applicants were "<6 years old," and the remaining $68.7 \%$ were "6-11 years old." More than half $(53.7 \%)$ of the applications were made by surgical branches, and $10.6 \%$ of the applications originated from branches that have pediatric subspecialty. The most frequently applying branches were "ophthalmology" (36.7\%), "physical medicine and rehabilitation" (18.2\%), and "dermatology" (15.3\%). In all applications overall, the most commonly encountered drug needed for OLDU was "botulinum toxin" (23.3\%), for which the most common diagnosis was cerebral palsy (58.9\%). Ophthalmologists were found to most frequently apply for adalimumab (35.7\%), where the most common indication for OLDU was iridocyclitis $(57.1 \%)$. Stratification by the age groups revealed also ophthalmologists to be the mostly applying PoPs in both "<6-year-old" and "6- to 11-year-old" groups (45.9\% and $32.6 \%$ respectively). While surgical branches were the predominant group $(\% 75,5)$ in applications for the "<6-year-old" group, internal branches were slightly higher (56.3\%) in "6- to 11-year-old" group. The most commonly applied drug for off-label use was ranibizumab (24.5\%) in "<6-year-old" group and "botulinum toxin" (27.9\%) in "6- to 11-year-old" group.

Conclusion(s): Our study indicates that OLDU in younger children by PoPs is mostly practiced from non-pediatric subspecialties, and approximately, three-quarters are concentrated in three specialty branches, namely, ophthalmology, physical medicine and rehabilitation, and dermatology. It is noteworthy that the need for OLDU by PoPs is more marked for "6- to 11-year-old" age group.

\section{REFERENCE(S)}

1. World Health Organization. Promoting Safety of Medicines for Children, 2007. ISBN: 9789241563437.

2. European Medicines Agency. Reflection Paper: formulations of choice for the paediatric population. Emea/Chmp/Peg/194810/2005. 2006.

\section{P174 | TP53 Genetic polymorphisms and susceptibility to cervical cancer in Bangladeshi women: A case-control study}

\author{
Mohd Nazmul Hasan $\mathrm{Apu}^{1}$; Md Shaki Mostaid ${ }^{2}$ \\ ${ }^{1}$ University of Dhaka; ${ }^{2}$ BRAC University
}

Introduction/Background \& Aims: TP53 is a well-known tumor susceptibility gene that plays a great role in modulating cell growth, maintenance, apoptosis, and DNA repair progression ${ }^{1}$. Two most common mutations of TP53 that occur at exon 4 are $\operatorname{Arg72Pro~}(\mathrm{G} \rightarrow \mathrm{C})$ and Pro47Ser ( $\rightarrow \mathrm{T}$ ), which are heavily linked to different types of carcinoma ${ }^{2,3}$. But extensive pharmacogenetic study of these two polymorphisms has not been conducted in cervical cancer. Therefore, the aim of this study was to assess the TP53 codon 72 and codon 47 polymorphisms and their relation to cervical cancer risk in Bangladeshi women.

Method/Summary of work: 100 cervical cancer patients and 98 agesex matched healthy controls were included from two institutions in Bangladesh. Polymerase chain reaction-restriction fragment length polymorphism (PCR-RFLP) method was used for genotyping two TP53 single nucleotide polymorphisms (codon 72 and codon 47) in patients and controls.

Results/Discussion: The results indicate that the TP53 Arg/Pro heterozygosity (adjusted $\mathrm{OR}=2.25,95 \% \mathrm{Cl}=1.14-4.43, p=0.019$ ), Pro/Pro mutant homozygosity (adjusted OR $=3.08,95 \%$ $\mathrm{Cl}=1.22-7.77, p=0.017)$, along with the combined genotype (Arg/Pro+Pro/Pro) (adjusted $\mathrm{OR}=2.48,95 \% \mathrm{Cl}=1.36-4.53$, $p=0.003$ ) significantly increases the risk of cervical cancer. Moreover, the cervical cancer patients with a first-degree relative cancer patient possesses 4.54 folds more risk ( $p=0.027$ ) of carrying a proline allele in codon 72 of the TP53 gene compared to those patients who does not have such relative. Finally, mutation in the codon 47 of the TP53 gene did not significantly increase the risk of cervical cancer in Bangladeshi women.

Conclusion(s): To conclude, this is the first study to identify that mutation in the TP53 codon 72 significantly increases the risk of cervical cancer in a female population in Bangladesh.

\section{REFERENCE(S)}

1. Sabapathy K, Lane DP. Therapeutic targeting of p53: all mutants are equal, but some mutants are more equal than others. Nat Rev Clin Oncol. 2018;15(1):13-30. https://doi.org/10.1038/nrclinonc.2017.151

2. Mostaid MS, Ahmed MU, Islam MS, Bin Sayeed MS, Hasnat A. Lung cancer risk in relation to TP53 codon 47 and codon 72 polymorphism in Bangladeshi population. Tumour Biol J Int Soc Oncodevelopmental Biol Med. 2014;35(10):10309-10317. https://doi.org/10.1007/s13277014-2285-2

3. Rivu SF, Apu MNH, Shabnaz S, et al. Association of TP53 codon 72 and $\mathrm{CDH} 1$ genetic polymorphisms with colorectal cancer risk in Bangladeshi population. Cancer Epidemiol. 2017;49:46-52. https://doi. org/10.1016/j.canep.2017.05.005

\section{P175 | Assessment of the effect of VKORC1 and CYP2C9 polymorphisms on warfarin dose requirements in Saudi patients: Interim results of a large cross-sectional study}

Salha Jokhab ${ }^{1}$; Farhad Kamali ${ }^{2}$; Maha Al-Rasheed ${ }^{3}$; Dana Makheet ${ }^{4}$; Peter Avery ${ }^{5}$; AbdulKareem Al-Momen ${ }^{6}$

${ }^{1}$ King Saud University-Newcastle University; ${ }^{2}$ Institute of Cellular Medicine, Newcastle University; ${ }^{3}$ Clinical pharmacy department, King Saud University; ${ }^{4}$ King Faisal Specialist Hospital \& Research Center; ${ }^{5}$ Schools of Mathematics, Statistics and Physics, Newcastle University; ${ }^{6}$ Department of Medicine, College of Medicine, King Saud University

Introduction: Warfarin is commonly used for the treatment and prevention of thromboembolic events. Genetics are reported to have an important effect on the inter-individual variability in warfarin dose requirement in various populations worldwide. ${ }^{1,2}$ However, the effect of genetic polymorphisms on warfarin dose requirement in Saudi population has not been fully investigated. Here, we present the results of an interim analysis of an ongoing large study investigating the prevalence of common polymorphisms in the genes that affect warfarin 
TABLE 1 Frequencies of VKORC1 -1639G > A and CYP2C9*2 and $* 3$ alleles polymorphisms

\begin{tabular}{llll}
$\begin{array}{l}\text { Genotype } \\
\text { VKORC1 -1639G > A }\end{array}$ & $\mathbf{N}(\%)$ & $\begin{array}{l}\text { Mean (SD) [range] } \\
\text { weekly doses }\end{array}$ & $\begin{array}{l}\boldsymbol{P} \\
\text { value }\end{array}$ \\
\hline GG & $20(32.3)$ & $48.2(21.3)[23.5-119]$ & 0.024 \\
\hline GA & $24(38.7)$ & $44.8(22.9)[12-98]$ & \\
\hline AA & $18(29.0)$ & $30.5(9.2)[21-49]$ & \\
\hline CYP2C9 & & & \\
${ }^{*} 1{ }^{*} 1$ & $18(29)$ & $48.5(25.2)[21-119]$ & 0.36 \\
${ }^{*} 1{ }^{*} 2,{ }^{*} 1{ }^{*} 3$ & $31(50)$ & $40.2(19.8)[21-98]$ & \\
${ }^{*} 2{ }^{*} 2,{ }^{*} 3 * 3,{ }^{*} 2{ }^{*} 3$ & $13(21)$ & $35.9(11.7)[12-49]$ & \\
\hline
\end{tabular}

pharmacology and metabolism and their impact on warfarin dose requirement in Saudi patients.

Aims: To reveal the distribution frequencies of allelic variants in VKORC1 and CYP2C9 genes in Saudi patients and the extent to which polymorphism in these genes affect warfarin dose requirements.

Methods: Patients aged 18 years or older stabilized on warfarin therapy were included. Real-time PCR were performed to investigate the frequency of the VKORC1 $-1639 \mathrm{G}>\mathrm{A}, \mathrm{CYP} 2 \mathrm{C} 9 * 2$ and $* 3$ alleles polymorphism.

Results: Table shows the frequencies of VKORC1 $-1639 \mathrm{G}>\mathrm{A}$, CYP2C9*2 and $* 3$ allelic variants in 62 Saudi patients and their effect on warfarin dose. Carriers of VKORC1 variant alleles require significantly lower warfarin doses than wild-type patients. Both homozygous and heterozygous CYP2C9 ${ }^{*} 2$ or ${ }^{*} 3$ carries require lower doses of warfarin than wild-type patients, but this did not reach statistical significance likely due to the interim small sample size.

Conclusion: The interim analysis results show that VKORC1 polymorphisms significantly affect warfarin dose requirements in Saudi patients. Polymorphisms in CYP2C9 gene are expected to have a significant effect on warfarin dose requirement, and it is anticipated that this can be demonstrated by data from a larger sample of patients.

\section{REFERENCE(S)}

1. Cooper, G. M., et al. (2008). A genome-wide scan for common genetic variants with a large influence on warfarin maintenance dose. Blood, 112(4), 1022-1027.

2. Tatarunas, V., et al. (2014). The effect of CYP2C9, VKORC1 and CYP4F2 polymorphism and of clinical factors on warfarin dosage during initiation and long-term treatment after heart valve surgery. Journal of Thrombosis and Thrombolysis, 37(2), 177-185.

\section{P177 | A retrospective study of gabapentinoid and anti-diabetic drug prescribing trends in Ireland over a 10-year period}

\section{Colette Morris; Helen Gallagher}

School of Medicine, University College Dublin
Introduction/Background \& Aims: Gabapentinoids are licensed in Ireland for the treatment of neuropathic pain(1). It has been reported internationally that the use and misuse of gabapentinoids is increasing (2). Gabapentinoids are recommended in the initial treatment of neuropathic pain associated with Type 2 diabetes mellitus (DM). The prevalence of Type 2 DM is increasing globally(3). The Health Service Executive (HSE) funds the supply of gabapentinoids through Primary Care Reimbursement Service (PCRS) schemes. The purpose of this study was to identify gabapentinoid and anti-diabetes medication prescribing trends in Ireland over a 10-year period.

\section{Method/Summary of work:}

- Prescribing frequency data were collected from PCRS Annual Reports.

- Data were gathered for "pregabalin," "gabapentin," and "Anatomical Therapeutic Chemical (ATC) Class A10: Drugs Used to Treat Diabetes."

- Results were analysed and presented using Microsoft Excel.

Results/Discussion: The total pregabalin prescribing frequency increased by $250 \%$ from 2008 to 2017 . The greatest relative increase was evident in the General Medical Services (GMS) scheme where pregabalin prescribing frequency increased by $360 \%$ from 2008 to 2017. There was a 338\% increase in Long Term Illness (LTI) scheme pregabalin prescriptions. The total gabapentin prescribing frequency increased by $36 \%$ from 2008 to 2017 . The greatest relative increase was observed in the LTI scheme where the frequency of gabapentin prescriptions increased by $102 \%$ from 2008 to 2017 . The total prescribing frequency of medications categorised as drugs used in diabetes increased by $66 \%$ from 2008 to 2017.

Conclusion(s): The increased use and circulation of gabapentinoids raise safety concerns regarding both their adverse effect profiles and abuse potential in vulnerable patients. Older patients are more susceptible to adverse effects of gabapentinoids which affect the nervous system, e.g., dizziness which increases the risk of falls(1). Given their abuse potential, gabapentinoids should be prescribed with caution in patients with a history substance abuse(1). This research quantifies the increased prescribing frequencies of pregabalin, gabapentin, and anti-diabetes medications in Ireland over 10 years. Further research is required to establish whether there is a relationship between the increased requirement for DM medications and the increased frequency of gabapentinoid prescribing in Ireland.

\section{REFERENCE(S)}

1. Health Products Regulatory Authority. Dublin 2, Ireland: Engine Solutions 2014 [Available from: http://www.hpra.ie/homepage/medicines/ medicines-information/find-a-medicine].

2. European Monitoring Centre for Drugs and Drug Addiction. European Drug Report 2018: Trends and Developments. Luxembourg: European Monitoring Centre for Drugs and Drug Addiction; 2018.

3. International Diabetes Federation. IDF Diabetes Atlas - 8th Edition. 2017. 


\section{P178 | Psychotropic medication prescribing for neuropsychiatric comorbidities in individuals diagnosed with autism spectrum disorder (ASD) in the UK}

Basmah Alfageh ${ }^{1}$; Kenneth Man ${ }^{1}$; Frank Besag ${ }^{1}$; Tariq Alhawassi ${ }^{2}$; Ian Wong ${ }^{1}$; Ruth Brauer ${ }^{1}$

${ }^{1}$ UCL; ${ }^{2}$ King Saud University

Introduction/Background \& Aims: Autism spectrum disorder (ASD) is a lifelong disorder. It impairs the quality of life of both the patients and their families. In the UK, risperidone is the only psychotropic medication approved for the management of the behavioural symptoms that may accompany autism disorder [1]. The aim of this study is to provide an evaluation of the changing trend in the incidence and prevalence of ASD in all age groups and to analyse the changing pattern of psychotropic medication prescribing in the UK.

Method/Summary of work: This is a population-based study of the annual incidence, prevalence, and psychotropic medication prescribing in patients with autism from 2009 to 2016. Neuropsychiatric conditions that are typically present in people with ASD include attention deficit hyperactivity disorder (ADHD), anxiety, behavioural/conduct disorders, intellectual disability, sleep disorders, depression, epilepsy, psychosis, and tic disorders. These conditions were identified in this cohort using data from The Health Improvement Network (THIN) which is an anonymised electronic health record of primary care in the UK.

Results/Discussion: 20,194 patients with an ASD diagnosis were identified over the study period. The prevalence of ASD increased 3.3-fold from 0.109 per 100 persons in 2009 to 0.355 per 100 persons in 2016. Approximately one-third of the identified cohort was prescribed at least one psychotropic medication. The most commonly prescribed psychotropic medications were hypnotics, antidepressants, antipsychotics, and stimulants. $32.1 \%$ of patients prescribed antipsychotic medication were on continuous therapy for more than one year. More than half of the total ASD cohort had at least one neuropsychiatric diagnosis in addition to autism. Behavioural/conduct disorders were diagnosed in $30.7 \%$ of the cohort.

Conclusion(s): The prevalence of ASD has been increasing over the past 20 years. Although the medications approved to manage the symptoms of ASD are limited, the prescribing of such medications is increasing. The long-term safety of these medications in people with ASD remains uncertain. Future studies on the impact of psychotropic medication use in patients with autism are needed.

\section{REFERENCE(S)}

1. World Health Organisation. Application for inclusion to the 19th expert committee on the selection and use of essential medicines: risperidone. 2013.

\section{P179 | The prevalence and incidence of dementia in people with diabetes}

\author{
Alaa Alsharif ${ }^{1}$; Li Wei ${ }^{2}$; Mansour Almetwazi ${ }^{3}$ Tian $\mathrm{Ma}^{2}$; \\ Rob Howard ${ }^{4}$; lan Wong ${ }^{2}$ \\ ${ }^{1}$ School of pharmacy, UCL; ${ }^{2}$ Research Department of practice and policy, \\ University College London School of Pharmacy, London, United Kingdom; \\ ${ }^{3}$ Clinical Pharmacy Department, College of Pharmacy, King Saud \\ University, Riyadh, Saudi Arabia; ${ }^{4}$ Division of Psychiatry, University \\ College London
}

Introduction/Background \& Aims: Few studies have shown an increased risk of dementia is associated with diabetes mellitus (1). The aim of this study is to estimate the prevalence and incidence of dementia in people with diabetes within primary care in the UK.

Method/Summary of work: Data from The Health Improvement Network (THIN) database in the UK were used for this descriptive study. Prevalent cases of dementia were individuals diagnosed with dementia and diabetes from 2000 to 2016. Incident cases were individuals who had not had a dementia diagnosis prior to the diagnosis of diabetes mellitus (DM). There were 19,052 prevalent cases. Rates were calculated annually, stratified by age and sex.

Results/Discussion: A total of 559,767 individuals had a diabetes diagnosis between 2000 and 2016, from these, 19,052 individuals were identified as having a dementia. The prevalence of dementia was $0.40 \%$ [95\% Cl $(0.40 \%-0.41 \%)]$ in 2000 and $2.4 \%[95 \% \mathrm{Cl}$ (2.32\% to $2.34 \%)$ in 2016 . The highest prevalence was in those aged $85+$ [from $2.9 \%$ in 2000 to $11 \%$ in 2016 , 95\% Cl $(2.89 \%$ 2.97\%) and (11\%-11.12\%)]. 269 and 1422 individuals developed dementia in 2000 and 2016, giving an incidence of 173 cases, 646 cases per 100,000 persons [95\% Cl (172-173) and (646-647) per 100,000 persons], respectively. Women had a higher prevalence and incidence of dementia than men $(3 \%$ vs $1.9 \%,[95 \% \mathrm{Cl}$ (2.88\%-3\%) and (1.8\%-2\%)] and 778 vs 545 cases per 100,000 persons in 2016, [95\% Cl (778-779) and (544-546) per 100,000 persons], respectively).

Conclusion(s): There was a trend of increasing prevalence and incidence of dementia in people with diabetes over the period of 2000 to 2016. This most likely reflects increasing recognition and diagnosis of dementia within the general population. This study adds to the evidence on dementia prevalence and incidence, particularly in the diabetic population.

\section{REFERENCE(S)}

1. Arvanitakis Z, Wilson RS, Bienias JL, Evans DA, Bennett DA. Diabetes mellitus and risk of Alzheimer disease and decline in cognitive function. Archives of neurology. 2004;61(5):661-6. 


\section{P181 | Efficacy of a $12 \mathrm{~h}$ intravenous acetylcysteine (SNAP) regimen following single acute paracetamol overdose stratified by risk of paracetamol-induced hepatotoxicity}

\author{
Amani Alrossies ${ }^{1}$; James Harnett ${ }^{2}$; David M. Wood ${ }^{3}$; Paul I. Dargan ${ }^{3}$; \\ Simon H.L. Thomas ${ }^{1}$; Ruben Thanacoody ${ }^{1}$ \\ ${ }^{1}$ Newcastle University; ${ }^{2}$ Guy's \& St Thomas NHS Foundation Trust \\ ${ }^{3}$ Faculty of Life Sciences and Medicine, King's College London
}

Introduction/Background \& Aims: A 2-bag $12 \mathrm{~h}$ acetylcysteine (NAC) regimen ("SNAP") causes fewer adverse reactions and appears to be similarly effective compared to the current 3-bag $21 \mathrm{~h}$ NAC regimen used for paracetamol poisoning(1). This research aimed to compare the efficacy of the SNAP regimen with the current regimen stratified by risk.

Method/Summary of work: The modified $12 \mathrm{~h}$ "SNAP" regimen (intravenous NAC $100 \mathrm{mg} / \mathrm{kg}$ over $2 \mathrm{~h}$ then $200 \mathrm{mg} / \mathrm{kg}$ over $10 \mathrm{~h}$ ) was introduced in 2016 in two UK hospitals for treatment of patients requiring NAC for paracetamol overdose. Data on efficacy outcomes (peak ALT and peak INR) were collected prospectively until 31st August 2019 and compared to data from audits of patients treated with the conventional $21 \mathrm{~h}$ regimen prior to October 2016. Patients with acute single paracetamol overdoses were categorised into 4 groups using 2 established risk factors for developing hepatotoxicity: admission paracetamol concentration above or below the 150-line on the paracetamol nomogram; time to acetylcysteine treatment $\leq 12$ or $>12 \mathrm{~h}$ from paracetamol ingestion. We determined the proportion of patients who developed a peak ALT $>1000$ or INR $>2$ in each risk category.

Results/Discussion: Patients treated $\leq 12 \mathrm{~h}$ post-ingestion and below the 150-line did not develop peak ALT>1000 with either NAC regimen. 2/372 (0.5\%) and 0/107 developed peak INR > 2 with the SNAP and conventional regimen respectively. In patients with paracetamol concentrations above the 150-line, peak ALT > 1000 occurred in $8 / 307(2.6 \%)$ and 5/115(4.3\%) with the SNAP and conventional regimen (absolute difference $-1.7 \%$; $95 \% \mathrm{Cl}-1.7$ to 7.3 ). Incidence of peak INR > 2 was $2.6 \%$ in both SNAP (8/307) and conventional (3/115) treatment groups (absolute difference 0; $95 \% \mathrm{Cl}-2.9$ to 4.9 ). In patients treated $>12 \mathrm{~h}$ post-ingestion with the SNAP and conventional regimens respectively, peak ALT $>1000$ occurred in $2 / 72$ (2.7\%) and $1 / 29$ (3.4\%) (absolute difference $-0.7 \% ; 95 \% \mathrm{Cl}-6.6$ - to 14.5 ) when paracetamol concentrations were below the 150-line and occurred in 11/87 (12.6\%) and 5/31 (16.2\%) of patients when paracetamol concentrations were above the 150 -line (absolute difference $-3.6 \%$; $95 \% \mathrm{Cl}-8.9$ to 20.9 ). In patients below the 150 -line, peak INR $>2$ occurred in $0 / 72$ and $1 / 29(3.4 \%)$ treated $>12$ h postingestion with the SNAP and conventional regimen; for those above the 150-line, the incidence of peak INR was $9 / 87$ (10.3\%) and 2/31 (6.5\%) respectively (absolute difference $3.8 \%, 95 \% \mathrm{Cl}-11$ to 13.2 ).
Conclusion(s): A simpler $12 \mathrm{~h}$ acetylcysteine regimen appears to be comparable in efficacy to the conventional regimen in all 4 risk groups but requires confirmation in larger cohorts of patients before implementation into clinical practice.

\section{REFERENCE(S)}

1. Pettie JM, Caparrotta TM, Hunter RW et al. Safety and Efficacy of the SNAP 12-hour acetylcysteine regimen for the treatment of paracetamol overdose. EClinicalMedicine 2019;11:11-17

\section{P182 | Examination of the adverse events in children and adults in primary care}

\author{
Volkan Aydin ${ }^{1}$; Omer Atac ${ }^{2}$; Narin Akici ${ }^{3}$; Ahmet Akici ${ }^{1}$ \\ ${ }^{1}$ Department of Medical Pharmacology, School of Medicine, Marmara \\ University, Istanbul, Turkey; ${ }^{2}$ Department of Public Health, School of \\ Medicine, Istanbul Medipol University, Istanbul, Turkey; ${ }^{3}$ Department of \\ Pediatrics, Haydarpasa Numune Training and Research Hospital, Istanbul, \\ Turkey
}

Introduction/Background \& Aims: Though mostly associated with hospital setting, drug-related adverse events (AEs) are also important in primary care, leading to worsening of symptoms and unplanned hospitalization in up to one-third of cases [1]. Several demographic characteristics are known to have an impact on the incidence of AEs in primary care practice [2]. We aimed to describe individual and clinical characteristics of the AEs in primary care setting.

Method/Summary of work: We reviewed a total of 2,947,112 encounters with single diagnosis in primary care centers of Istanbul province in 2016 . The centers were selected by systematic sampling and prescriptions with diagnosis including "drugs, medicaments, and biological substances causing adverse effects in therapeutic use (ICD Code: Y40 to Y59)" were examined with respect to its details on sex, age group (children: <18-year-old, adults: $\geq 18$-year-old), and drug utilization parameters.

Results/Discussion: We identified 972 AEs among all prescriptions with single diagnosis (33.0 per 100,000 encounters). Most of these AEs $(60.7 \%)$ were detected in females and near half in children (49.5\%). The number of AEs was 54.5 and 23.8 (per 100,000 encounters) in children and adults. Per 100,000 encounters, while the number of AEs was higher in boys (58.8) than that in girls (49.7) in children, it was 30.3 and 13.8 in female and male adults, respectively. In total population, we detected these AEs to be most commonly related with "anti-megaloblastic-anemia preparations" (41.0\%) and "vaccine and other biological substances" (38.0\%). While $97.8 \%$ of the latter were observed in children, AEs related with "agents primarily affecting blood constituents" were mostly (95.5\%) found in adults. Primary care approach to manage these AEs showed that the number of drugs per encounter was lower in children $(1.16 \pm 0.39)$ than that in adults $(1.32 \pm 0.57)(p=0.02)$. In this primary care management, around half of the prescriptions (50.2\%) contained vitamins and $44.7 \%$ had at least an analgesic preparation. Vitamin-containing encounters were found 
to be mostly prescribed for adults (76.2\%) whereas analgesiccontaining encounters were predominantly prescribed for children (95.4\%).

Conclusion(s): Our study regarding AEs in primary care practice shows higher incidence in children mostly due to "vaccine and biological products" and managed with analgesics. AEs experienced by adults appear to mainly affect female patients. Available findings reflect differential patterns of AEs between children and adults in primary care setting.

\section{REFERENCE(S)}

1. Wetzels R, Wolters R, van Weel $C$ et al. Harm caused by adverse events in primary care: a clinical observational study. J Eval Clin Pract 2009;15:323-7.

2. TTsang C, Bottle A, Majeed A, Aylin P. Adverse events recorded in English primary care: observational study using the general practice research database. Br J Gen Pr. 2013;63:e534-42.

\section{P183 | Prevalence of potential drug-drug interactions during hospital discharge in a tertiary care center in Nepal}

\author{
Bijay Bhandari; Pratik Lamichhane; Dipendra Yadav; \\ Sangha Ratna Bajracharya \\ Institute of Medicine, Tribhuvan University
}

Introduction/Background \& Aims: In our setup, potential drug-drug interactions are overlooked in routine clinical practice. In general, most of the discharge are handwritten in the developing world, and the discharge prescriptions are not checked with the database for potential drug-drug interactions checker.

Patients are not under the direct supervision of healthcare professionals after the discharge in Nepal. Treatment inefficacy or suboptimal treatment and adverse drug reaction due to drug-drug interactions can increase the duration of treatment ultimately leading to increase healthcare cost [1]. Therefore, this study aims to find out the prevalence and anticipate potential drug-drug interaction in the prescribed drugs in clinical practice.

Method/Summary of work: A cross-sectional study was conducted in the Department of Internal Medicine, in a University Teaching hospital in Kathmandu, Nepal from Oct 2018 to Dec 2018. Ethical approval was taken from IRB-Institute of Medicine, Kathmandu, Nepal.

Through simple random sampling, the data about drug prescription was collected from the patient discharged record, from different
Units of the Department of Internal Medicine. The sample size was calculated using $50 \%$ prevalence at a $95 \%$ confidence interval with a $5 \%$ margin of error and $10 \%$ nonresponse rate.

A data abstraction form was developed and used to collect the data and was transferred to electronic format after verifying. The handwritten prescription, where spellings were illegible, was clarified from the prescribers of the concerned unit. Information on discharge prescriptions was retrieved from the hospital discharge letter. Demographic information such as age, sex, main diagnoses (according to ICD-10 classification), and drugs were classified according to the anatomical therapeutic chemical (ATC) classification. Potential drug-drug interactions ( $p D D I s)$ were checked by using Lexicomp ${ }^{\circledR}$ drug interactions. The severity of pDDIs was classified as Major, Moderate, and Minor according to per Lexicomp ${ }^{\circledR}$. The data were analyzed using Statistical Package for Social Science (SPSS) version 22 for windows for descriptive statistics.

Results/Discussion: We collected 401 discharge prescriptions, out of which 19 were invalidated due to exclusion criteria. We analyzed 382 discharge prescriptions, out of which the total prevalence of potential drug-drug interactions was $78.27 \%$ (74.23-82.30) with $95 \%$ $\mathrm{Cl}$. The Mean age of the population was $53.8 \pm 20$ years and the Mean number of medications was 6.3 and the Mean duration of hospital stay was $7.9 \pm 5.7$ days and the average number of interactions equal to 3.9. Severity rating as major, moderate, and minor pDDIs was found to be $10.7 \%, 76.5 \%, 11.7 \%$ respectively. Among different units of Internal Medicine, patients discharged from Cardiology ward were in the top with $36.7 \%$ while it was around half of this (19.1\%) in Pulmonology discharged prescriptions with Gastroenterology unit (7\%) being least in the row. In patients with 5 drugs, pDDIs were $6.91 \%$ while with 7 drugs pDDIs were found to be more than double (14.41\%).

Conclusion(s): There is a high prevalence of pDDIs in the Internal Medicine wards in a tertiary care center in Nepal. Use of drug-drug interactions checker database before discharge with computer-based discharge prescriptions are recommended. The findings from this study will help in increasing awareness of pDDIs. This baseline study can lead to further prospective studies to observe clinically evident drug-drug interactions outcome.

\section{REFERENCE(S)}

1. Shetty V, Chowta M N, Chowta N K, Shenoy A, Kamath A, Kamath P. Evaluation of potential drug-drug interactions with medications prescribed to geriatric patients in a tertiary care hospital. Journal of Aging Research. 2018 Oct 09;2018:1-6. https://doi.org/10. $1155 / 2018 / 5728957$

TABLE 1

\begin{tabular}{|c|c|c|c|c|c|c|}
\hline Severity of interactions & Cardiology & Nephrology & Pulmonology & Neurology & Gastroenterology & Total (\%) \\
\hline Major & $52(3.4 \%)$ & $20(1.3 \%)$ & $36(2.4 \%)$ & $39(2.6 \%)$ & $16(1.1 \%)$ & $163(10.7)$ \\
\hline Minor & $54(3.6 \%)$ & $56(3.7 \%)$ & $31(2.0 \%)$ & $26(1.7 \%)$ & $11(0.7 \%)$ & $178(11.7)$ \\
\hline Moderate & 449 (29.6\%) & $264(17.4 \%)$ & $217(14.3 \%)$ & $152(10 \%)$ & $80(5.3 \%)$ & $1162(76.5)$ \\
\hline No interactions identified & $2(0.1 \%)$ & $4(0.3 \%)$ & $6(0.4 \%)$ & $4(0.3 \%)$ & $0(0 \%)$ & $16(1.1)$ \\
\hline Total interactions & $557(36.7 \%)$ & $344(22.6 \%)$ & $290(19.1 \%)$ & $221(14.5 \%)$ & $107(7 \%)$ & 1519 (100) \\
\hline
\end{tabular}


Oral Communications, Monday 16th December, 14:30

\section{Clinical Oral Communications 1}

\section{OC039 | An open-label, randomized, single- dose, comparative pharmacokinetics study of YLB113 and the etanercept reference product in healthy adult male subjects}

Ahmad Al-Ghazawi ${ }^{1}$; Santhosh Khirasagar ${ }^{2}$; Ravi Sekhar Kasibhatta²; Neelima Godse ${ }^{2}$; Ahmad Al-Ghazawi ${ }^{1}$; Dhananjay Bakhle ${ }^{2}$

${ }^{1}$ Triumpharma; ${ }^{2}$ Lupin Limited

Introduction/Background \& Aims: YLB113 is being developed as a biosimilar of the anti-tumor necrosis factor etanercept reference product (RP), Enbrel ${ }^{\circledR}$, which is approved for the treatment of moderate-to-severe rheumatoid arthritis and other chronic, immunemediated, inflammatory diseases. An open-label, balanced, 2-sequence, 2-treatment, crossover, pharmacokinetic (PK) study was conducted to compare the relative bioavailability, safety, and tolerability of YLB113 and the RP in healthy male volunteers.

Method/Summary of work: Healthy, adult male subjects aged 18 to 50 years were randomized to receive a single subcutaneous dose of YLB113 (test product) in Period 1 and the RP in the Period 2. A washout period of 28 days separated the 2 doses (Figure 1). Blood samples were collected predose and until 480 hours postdose in a staggered manner for PK analysis in both periods.

Results/Discussion: A total of 52 subjects were enrolled; 51 subjects were dosed during Period 1 and completed the period, whereas 43 subjects were dosed during Period 2 and completed the study. The $90 \%$ confidence intervals for the ratios of test and RP averages (least squares mean) derived from the analysis of log-transformed PK parameters, $C_{\max }, \mathrm{AUC}_{(\mathrm{O}-\mathrm{t})}$, and $\mathrm{AUC}_{(\mathrm{O}-\infty)}$ for etanercept were between the limits of $80.00 \%$ and $125.00 \%$ (Table). Thus, the test product YLB113 met the bioequivalence criteria. A total of 24 subjects reported 53 adverse events, including 42 mild and 11 moderate events. Treatment-emergent adverse events were reported by 14 and 16 subjects following administration of YLB113 and the RP, respectively. Based on the safety profile, YLB113 and the RP were well tolerated.

$\mathrm{AUC}_{\mathrm{O}-\infty}$ indicates area under the plasma concentration time curve from 0 to infinity; $\mathrm{AUC}_{0-\mathrm{t}}$, area under the plasma concentration time curve from $\mathrm{O}$ to the last detectable value; $\mathrm{Cl}$, confidence interval; $\mathrm{C}_{\max }$, maximum concentration; $\mathrm{CV}$, coefficient of variation; $\mathrm{PK}$, pharmacokinetic.

Conclusion(s): These results indicate that a single dose of YLB113 exhibits PK and safety profiles comparable with the RP in healthy adult male subjects, and the risk-benefit profile of etanercept was equivalent to the favorable risk-benefit profile of the innovator product.
Test (A)

YLB113 (Etanercept)

$50 \mathrm{mg}$ Solution for

Injection in pre-filled

syringes for

subcutaneous use

(AY Pharmaceuticals Co. Japan)

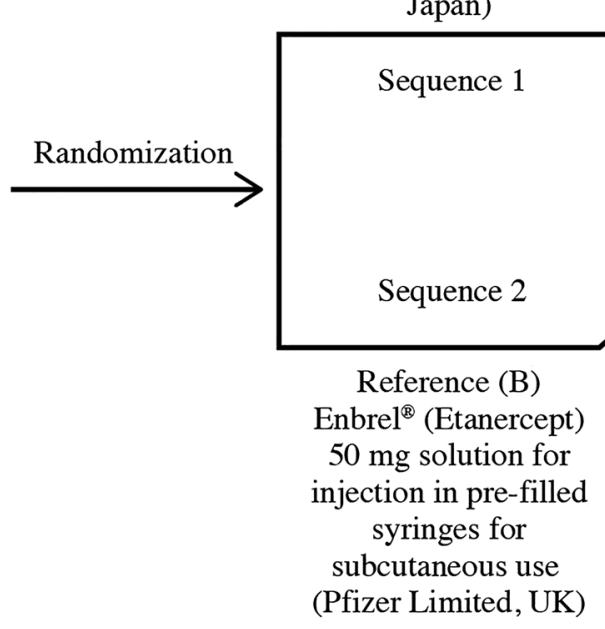

Test (A)

YLB113 (Etanercept)

$50 \mathrm{mg}$ Solution for Injection

in pre-filled syringes for subcutaneous use (AY Pharmaceuticals Co. Japan)

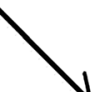
Washout period

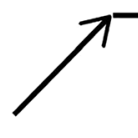

Sequence 2 -28 days

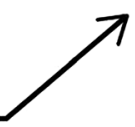

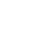
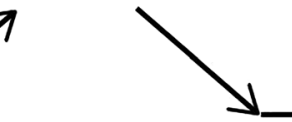

Sequence 1

Reference (B) Enbrel $^{\circledR}$ (Etanercept) $50 \mathrm{mg}$ solution for injection in pre-filled syringes for subcutaneous use (Pfizer Limited, UK)
Final Examination 
TAB LE 1 Statistical comparisons of etanercept pharmacokinetic parameters

\begin{tabular}{|c|c|c|c|c|c|c|c|}
\hline \multirow[t]{2}{*}{ Primary PK parameter } & \multirow[t]{2}{*}{ Intra-subject variability CV } & \multicolumn{2}{|c|}{ Geometric means } & \multirow[t]{2}{*}{ Ratio (\%) } & \multicolumn{2}{|c|}{$90 \% \mathrm{Cls}$ of the ratio } & \multirow[t]{2}{*}{ Power } \\
\hline & & Test $A(N=43)$ & Reference $B(N=43)$ & & Lower & Upper & \\
\hline $\mathrm{C}_{\max }(\mathrm{ng} / \mathrm{ml})$ & 24.38 & 2873.88 & 2884.39 & 99.64 & 91.31 & 108.72 & 99.02 \\
\hline $\mathrm{AUC}_{\mathrm{O-t}}\left(\mathrm{ng}{ }^{*} \mathrm{hr} / \mathrm{ml}\right)$ & 20.46 & $463,705.43$ & $487,979.40$ & 95.03 & 88.29 & 102.27 & 98.72 \\
\hline $\mathrm{AUC}_{0-\infty}\left(\mathrm{ng}^{*} \mathrm{hr} / \mathrm{ml}\right)$ & 19.99 & $485,443.45$ & $512,892.18$ & 94.65 & 88.08 & 101.70 & 98.70 \\
\hline
\end{tabular}

OC040 | Vortioxetine as adjunctive therapy to risperidone for treatment of patients with chronic schizophrenia: A randomized, doubleblind, placebo-controlled trial

Shahin Akhondzadeh; Samaneh Bayanati; Ehsan Mozen-Zadeh

Tehran University of Medical Sciences

Introduction/Background \& Aims: Schizophrenia is a chronic psychiatric disorder accompanied by high rates of morbidity, and mortality and high burden of disease. In order to increase response to antipsychotic treatments and improve negative symptoms in patients with schizophrenia, several different strategies have been studied, among which, add-on antidepressant therapy is both an active line of research and a common practice worldwide (1). Vortioxetine is a novel antidepressant which appears to be an interesting candidate for adjunctive therapy in schizophrenia. We aimed to assess the efficacy and adverse events of vortioxetine, as an adjunctive therapy to antipsychotics, in treatment of patients with stable schizophrenia, using a randomized clinical trial design. Our primary interest was the effect of vortioxetine on negative symptoms, with the assessment of positive, general psychopathology, and total symptoms as our secondary goal.

Method/Summary of work: This was an 8-week, randomized, doubleblind, placebo-controlled, parallel-group clinical trial of vortioxetine in patients with schizophrenia, who were referred to a large-scale academic psychiatry hospital (Roozbeh Hospital, Tehran University of Medical Sciences, Tehran, Iran) from November 2017 to April 2019. The study was registered at the Iranian Registry of Clinical Trials after approval by the Institutional Review Board of TUMS in accordance with the World Medical Association code of ethics (IR.TUMS.VCR. REC.1396.3556). The patients were assessed using the positive and negative syndrome scale (PANSS), extrapyramidal symptom rating scale, and Hamilton depression rating scale during the study course. All participants had PANSS negative symptoms subscale score of $\geq 17$ at baseline. To be eligible, treatment with a stable dose of risperidone for a minimum of eight weeks was required prior to entry. Patients were also required to be clinically stable for a minimum of four weeks prior to the study. Patients were assigned to risperidone up to $6 \mathrm{mg} /$ day during the course of the trial. Vortioxetine $10 \mathrm{mg}$ twice daily or placebo were started after patients received risperidone for 8 weeks and stabilized. Two-way repeated measure analysis of variance (ANOVA) was used for assessment of time $\times$ treatment interaction effects with the measurement time-points and the treatment groups as the between-subject and within-subject factors, respectively.

Results/Discussion: Sixty-eight patients completed the trial. There was a significantly more negative symptoms score reduction from baseline to endpoint in the vortioxetine arm accompanied by a large effect size (Mean difference $(\mathrm{Cl} 95 \%)=-1.82(-2.73$ to -0.92$)$; Cohen's $d(\mathrm{Cl} 95 \%)=0.97(0.47$ to 1.48$))$. Also, a significant time $\times$ treatment interaction effect was observed between the two treatment arms [two-way ANOVA with Greenhouse-Geisser correction: $F(d f$, mean square $)=10.45(1.97,18.39), p=-0.000]$. In addition to, There was a significant difference in total score reduction from baseline to endpoint, favoring the vortioxetine arm accompanied by a large effect size (Mean difference $(\mathrm{Cl} 95 \%)=-2.09(-3.16$ to -1.01$)$; Cohen's $d(\mathrm{Cl} 95 \%)=0.95(0.45$ to 1.45$))$. Moreover, a significant time $\times$ treatment interaction effect was observed between the two treatment arms [two-way ANOVA with Greenhouse-Geisser correction: $F(d f$, mean square $)=11.72(1.91,27.37), p=0.000]$. No significant difference was detected for changes in positive symptoms score or PANSS general psychopathology score as the other secondary outcomes from baseline to endpoint between the two treatment arms. Incidence of adverse events was comparable between groups. To the best of our knowledge, this is the first report of treatment with vortioxetine in patients with schizophrenia. Considering that vortioxetine is classified as an atypical antidepressant with a unique and complex mechanism of action but relatively similar clinical effects as other antidepressants, our findings are better compared with the bulk of evidence on efficacy of antidepressants in treatment of patients with schizophrenia, rather than being compared to any specific medication or single clinical trial.

Conclusion(s): This study provided the first evidence on potential therapeutic effects of vortioxetine, as an adjunctive antidepressant to antipsychotics, in treatment of patients with schizophrenia. Future comprehensive studies are warranted to confirm our findings.

\section{REFERENCE(S)}

1. Galling B, Vernon JA, Pagsberg AK, Wadhwa A, Grudnikoff E, Seidman AJ, Tsoy-Podosenin M, Poyurovsky M, Kane JM, Correll CU, (2018) Efficacy and safety of antidepressant augmentation of continued antipsychotic treatment in patients with schizophrenia. Acta Psychiatr Scand 137(3), 187-205. 


\section{OC041 | The topical anti-microbial peptide omiganan recovers dysbiosis but does not improve clinical symptoms in patients with mild to moderate atopic dermatitis}

Salma Assil ${ }^{1}$; Tessa Niemeyer-Van der Kolk ${ }^{1}$; Thomas Buters ${ }^{1}$; Martijn Van Doorn ${ }^{2}$; Jacobus Burggraaf ${ }^{1}$; Robert Rissmann ${ }^{1}$

${ }^{1}$ Centre for Human Drug Research; ${ }^{2}$ Erasmus Medical Centre

Background: in a previous phase II trial in 36 patients with mild to moderate $A D$, omiganan an indolicidin analogue with antimicrobial and immunomodulatory properties significantly reduced target lesion oSCORAD and morning itch and improved target lesion dysbiosis by reducing Staphylococcus and increasing diversity.

Objective: to explore clinical efficacy and pharmacodynamics of omiganan with an optimized dose regimen in a larger patient population.

Methods: in total 80 patients with mild to moderate $A D$ were enrolled in this single-center, phase II randomized, double-blind, placebo-controlled trial. Patients were randomized 1:1:1:1 to omiganan $1 \%$, omiganan $1.75 \%$, omiganan $2.5 \%$, or vehicle gel. The study drug was applied to all AD lesions once daily for 28 consecutive days. Efficacy was evaluated by EASI, oSCORAD, and IGA. One AD lesion was assigned as "target lesion" for various pharmacodynamic assessments.

Results: A reduction of target lesions $S$. aureus was observed in all active treatment groups in the culture, while an increase was apparent in the vehicle group. This reduction was statistically significant for omiganan $2.5 \%$ treatment compared to vehicle $(p=0.0249)$. All active treatments normalized the microbiome by reducing Staphylococcus genus and increased diversity index. No substantial improvement of AD was observed compared to vehicle.

Conclusions: Recovery of the target lesion dysbiosis by a reduction of S. aureus in culture and Staphylococcus genus in the microbiome was observed in all active treatment groups, while this did not lead to clinical improvement of the lesions. This finding weakens the suggested major role of the microbiome in the pathogenesis of $A D$.

\section{REFERENCE(S)}

1. Totte JE, van der Feltz WT, Hennekam M, van Belkum A, van Zuuren EJ, Pasmans SG. Prevalence and odds of Staphylococcus aureus carriage in atopic dermatitis: a systematic review and meta-analysis. $\mathrm{Br}$ J Dermatol. 2016;175(4):687-95.

2. Geoghegan JA, Irvine AD, Foster TJ. Staphylococcus aureus and atopic dermatitis: a complex and evolving relationship. Trends Microbiol. 2018; 26(6):484-97.

3. de Wit J, Totte JEE, van Mierlo MMF, van Veldhuizen J, van Doorn MBA, Schuren FHJ, et al. Endolysin treatment against Staphylococcus aureus in adults with atopic dermatitis: a randomized controlled trial. The Journal of allergy and clinical immunology. 2019.

\section{Oc042 | Serotonin syndrome induced by drug-drug interactions including tramadol: $A$ systematic review}

Charlotte Fouque ${ }^{1}$; Cécile Conte ${ }^{1}$; DDI-vigilance study group ${ }^{2}$; Maryse Lapeyre-Mestre ${ }^{1}$

${ }^{1}$ INSERM, Université de Toulouse, Centre Hospitalo-Universitaire de Toulouse (CHU Toulouse), UMR, 1027, Toulouse, France; ${ }^{2}$ Sorbonne Université, INSERM, Institut Pierre Louis d'épidémiologie et de Santé publique, Université Paris 13, Laboratoire d'informatique médicale et d'ingénierie des connaissances en e-santé, Assistance Publique - Hôpitaux de Paris (APHP), Université de Versailles Saint-Quentin-en-Yvelines

Introduction/Background \& Aims: Serotonin syndrome (SS) is a potential life threatening adverse drug reaction (ADR) due to an excessive serotoninergic transmission characterized by neuromuscular excitation, dysautonomia and mental status changes. SS occurs following the administration of serotoninergic drugs alone or following pharmacodynamics or pharmacokinetic drug-drugs interactions (DDI). Tramadol is a weak opioid analgesic drug with serotoninergic properties widely used in acute or chronic pain by people of any age. The description of DDI with tramadol and clinical context leading to SS is crucial to identify patients but also circumstances more at risk and to promote proper use of this drug. Prevalence data are also lacking. The aims of study were to identify drug interactions with tramadol inducing SS and to describe prevalence, type and context of interactions (acute or chronic exposure, profile of patients at risk ...).

Method/Summary of work: A systematic review was conducted in accordance with Preferred Reporting Items for Systematic Reviews and Meta-Analyses (PRISMA) guidelines and was registered in PROSPERO (CRD42018105581). The search strategies in MEDLINE and WEB OF SCIENCE included terms relating to "tramadol," "serotonin syndrome," and "serotonin agent" until 20/06/2018.

Results/Discussion: The literature search identified 543 articles. After selection process, 48 published articles were included: 29 case reports/case series, 16 narrative or systematic reviews and 5 observational studies. Drugs mainly involved in drug interactions with tramadol leading to SS were antidepressants $(n=26)$. The addition of another opioid drug to tramadol was also described in 5 cases. Interactions with tramadol occurred with another serotoninergic drug $(n=16$ cases), two serotonergic drugs ( $n=11$ cases) or even three drugs ( $n=2$ cases). Patients included in case reports were mostly women $(n=17)$ with a mean age of $60.2(+/-14.8)$ years. Most patients were comorbid. Tramadol was prescribed in a context of acute pain $(n=13$ cases) or chronic pain ( $\mathrm{n}=9$ cases). SS onset time was rapid (<1 month). This ADR was serious with hospitalization or lifethreatening conditions. Observational studies depicted a prevalence of drug interactions that could lead to SS between $2 \%$ and $8 \%$ according to data sources.

Conclusion(s): Our review established a list of drugs interaction with tramadol leading to SS with a better understanding of SS context in routine practice. Some publications show high prevalence of 
concomitant use of serotoninergic drugs in health databases with no data on the prevalence of SS after these co-prescriptions.

\section{REFERENCE(S)}

1. Abadie D, Rousseau V, Logerot S, Cottin J, Montastruc JL, Montastruc F. Serotonin syndrome: analysis of cases registered in the French pharmacovigilance database. J Clin Psychopharmacol. 2015 Aug; 35(4):382-8.

2. Machado-Alba JE, Morales-Plaza CD. Prevalence of avoidable potential interactions between antidepressants and other drugs in Colombian patients. Rev Colomb Psiquiatr. 2013 Jun;42(2):162-6.

3. Ringland C, Mant A, McGettigan P, Mitchell P, Kelman C, Buckley N, Pearson SA. Uncovering the potential risk of serotonin toxicity in Australian veterans using pharmaceutical claims data. $\mathrm{Br} J \mathrm{Clin}$ Pharmacol. 2008 Nov;66(5):682-8.

\section{OC043 | Patterns of strong opioid use and potential inappropriate use in cancer patients during the last year of life In France}

\author{
Maryse Lapeyre-Mestre ${ }^{1}$; Thanh Hang $\mathrm{Chu}^{2}$; Manuela Rueter ${ }^{3}$; \\ Berangere Baricault ${ }^{3}$; Aurore Palmaro ${ }^{1}$ \\ ${ }^{1}$ University of Toulouse; ${ }^{2}$ Hanoi University of Pharmacy; ${ }^{3}$ Toulouse \\ University Hospital
}

Introduction/Background \& Aims: A better knowledge of opioid prescribing patterns and whether prescriptions are in concordance with the current recommendations can help identify areas of potential improvement in cancer pain management. Studies investigating patterns of strong opioid prescribing and potentially inappropriate use (PIU) in cancer outpatients remain scarce $(1,2)$. This study aimed to identify and quantify the strong opioid patterns of use and potential inappropriate use (PIU) in cancer patients in their last year of life.

Method/Summary of work: A cohort of adult cancer patients died between 2011 and 2014 and exposed to strong opioid analgesics during the 12 months before death were identified in the "Echantillon Généraliste de Bénéficiaires" (EGB, a 1/97th random sample of the French general population) was identified (3). Potentially inappropriate prescription was defined by (a) overlapping prescriptions; (b) contraindicated prescriptions; (c) lack of laxatives with chronic treatments; (d) potential drug interactions; (e) prescription in patients hospitalized for opioid-related disorders within the period. Factors associated with this PIU were investigated through logistic regression models.

Results/Discussion: The cohort consisted of 2,236 patients (median age 72 years (IQR: 61-82), 44.1\% of women). PIU (mainly represented by the lack of laxative co-prescription, lacking/insufficient morphine background treatment for transmucosal fentanyl, and overlapping prescriptions) significantly decreased from $37.6 \%$ (2011) to $29.8 \%$ (2014, $p=0.006$ ). PIU was associated with younger age (adjusted Odds Ratio $[\mathrm{aOR}]=0.99 ; 95 \% \mathrm{Cl}$ : 0.98-0.99); social deprivation (aOR = 1.77; 95\% $\mathrm{Cl}: 1.01-3.10)$, cancer of the upper aerodigestive tract $(\mathrm{aOR}=1.93$; $95 \% \mathrm{Cl}: 1.14-3.28)$ and digestive organs $(\mathrm{aOR}=1.78 ; 95 \% \mathrm{Cl}$ : 1.37-2.78), fentanyl prescription ( $\mathrm{aOR}=2.36$; $95 \% \mathrm{Cl}$ : $1.86-3.00)$ or in combination with morphine ( $\mathrm{aOR}=1.68$; 95\% Cl: 1.17-2.39), other strong opioids (buprenorphine, hydromorphone) ( $\mathrm{aOR}=2.04 ; 95 \% \mathrm{Cl}$ : 1.29-3.23) and prior strong opioid prescription $(\mathrm{aOR}=1.88 ; 95 \% \mathrm{Cl}$ : 1.50-2.36).

Conclusion(s): In France, one of three cancer patients exposed to strong opioids experienced PIU and this proportion tended to decrease over time, whereas PIU of transmucosal fentanyl remained stable. There is still room for progress in cancer pain management at the end of life.

\section{REFERENCE(S)}

1. Ziegler L, Mulvey M, Blenkinsopp A, Petty D, Bennett MI. Opioid prescribing for patients with cancer in the last year of life: a longitudinal population cohort study. Pain. 2016;157:2445-51.

2. Gao W, Gulliford M, Bennett MI, Murtagh FE, Higginson IJ. Managing cancer pain at the end of life with multiple strong opioids: a population-based retrospective cohort study in primary care. PloS one. 2014;9:e79266.

3. Moulis G, Lapeyre-Mestre M, Palmaro A, Pugnet G, Montastruc JL, Sailler L. French health insurance databases: what interest for medical research? La Revue de medecine interne. 2015;36:411-7.

\section{Clinical Oral Communications 2}

\section{OC044 | Antimicrobial prescribing in New Zealand: Medical versus non-medical prescribing practices}

\author{
Betty Poot ${ }^{1}$; Katherine Nelson ${ }^{1}$; Denise Taylor ${ }^{1}$; Mark Weatherall ${ }^{2}$ \\ ${ }^{1}$ Victoria University Wellington; ${ }^{2}$ University of Otago, Wellington
}

Introduction/Background \& Aims: New Zealand's (NZ) level of antimicrobial resistance is low compared to other countries; however, the use of antibiotics in community-based patients has increased. The impact of resistance to antibiotics may be reduced by improving awareness and understanding of appropriate prescribing and for this it is important to understand the antimicrobial prescribing practices in different health professional prescribing groups. This study aims to describe antibiotic agents dispensed in community settings in NZ and compare this amongst different prescriber groups.

Method/Summary of work: A retrospective cohort study of routinely collected data of community dispensed medicines for 2018 from a search of the NZ Ministry of Health Pharmaceuticals Collection claims database. Data included dispensed medicines, patient demographics and deprivation index, and prescribers' professional group. The prescriber groups included 1000 randomly selected dentists, 1000 medical doctors, all nurse prescribers (nurse practitioner [NP], registered nurse $[R N]$ prescriber and community nurse prescriber) and all pharmacist prescribers.

Results/Discussion: There were 2,028,571 unique patient-medication combinations; with 328,284 (16.1\%) patient-antibacterial agent 
combinations. In all, 362,447/622,224 (58.3\%) patients received an antibiotic prescription. Dentists prescribed the highest proportion of antibiotic agents to total medicines (60.8\%); followed by community nurse prescribers (24.9\%), NPs (14.0\%), medical doctors (13.2\%), RN prescribers (12.1\%), and pharmacists (1.1\%). The most commonly dispensed antibiotic agents were amoxicillin (100,363, 30.6\%), amoxicillin with clavulanic acid $(46,122,14.1 \%)$, flucloxacillin $(27,785$, 8.5\%), metronidazole (15,247, 4.6\%), doxycycyline (14,494, 4.4\%), cefalexin $(12,750,3.9 \%)$ and trimethoprim $(10,833,3.3 \%)$. Nurses were less likely to prescribe amoxicillin with clavulanic acid as a proportion of total medications; RR 0.083 and 0.63 , compared to dentists and doctors, $P<0.001$. Patients who were dispensed antibiotics were more likely to be Māori or Pasifika $47.2 \%$ and $47.5 \%$ compared to European ethnicity $40.1 \%$. Nurses prescribed to the more non-European populations Māori or Pasifika (30.6\%) compared to dentists $(16.9 \%)$ or medical doctors $(22.6 \%)$ and to those who lived more deprived areas, mean (SD) NZ Deprivation index 6.56 (2.76) compared to dentists, 5.48 (2.87) and medical doctors, 5.83 (2.89).

Conclusion(s): Greater than one third of patients are dispensed antibacterial agents. There are some differences in prescribing patterns between prescriber groups. Medical doctors and dentists were more likely to prescribe the more broad-spectrum antibacterial agents than nurse prescribers. Some differences in what is prescribed and to whom can be explained by the nature of the prescriber's scope of practice and regulatory restrictions on medicines for specific prescribing groups.

\section{OC045 | Multisource comprehensive review on whether ibuprofen exacerbates bacterial infections}

Thomas Soeiro $^{1}$; Delphine Bourneau-Martin ${ }^{2}$; Joëlle Micallef ${ }^{1}$; Annie-Pierre Jonville-Béra ${ }^{3}$

${ }^{1}$ Centre régional de pharmacovigilance, Service de pharmacologie clinique, Centre hospitalo-universitaire de Marseille, France; ${ }^{2}$ Centre régional de pharmacovigilance, Service de pharmacologie clinique, Centre hospitalo-universitaire d'Angers, France; ${ }^{3}$ Centre régional de pharmacovigilance, Service de pharmacosurveillance, Centre hospitalouniversitaire de Tours, France

Introduction/Background \& Aims: All adverse effects registered by the network of French Pharmacovigilance Centers (FPC) are medically and pharmacologically validated, and are monitored to ensure informativeness. As France is one of the top three contributors of VigiBase [1], French cases are a first-class source for generating and confirming signals of pharmacovigilance. Prompted by noteworthy new cases, this multisource comprehensive review assessed whether oral ibuprofen treatment for fever or pain contributes to exacerbate skin and soft tissue, or pleuropulmonary infections in immunocompetent adults and children.

Method/Summary of work: Four complementary data sources were analyzed up to 2018 for skin and soft tissue, or pleuropulmonary infections under ibuprofen: (i) Serious cases registered by the FPC; (ii) Automated signal detection from the database of FPC, EudraVigilance, and VigiBase; (iii) Pharmacoepidemiological studies reporting a measure of association; (iv) In vitro and in vivo experimental studies.

Results/Discussion: This multisource comprehensive review identified 242 serious cases registered by the FPC, among which infections often occurred in children during short ibuprofen treatment including when antibiotherapy is associated (Table 1). Automated signal detection was positive for most infections studied in the database of FPC, EudraVigilance, and VigiBase. Most pharmacoepidemiological studies, whatever the method and the country, suggested that exposure to NSAID was associated to increased risk of serious skin and soft tissue, or pleuropulmonary complications (Table 2). In vivo and in vitro experimental studies suggested that NSAIDs disrupt resolution of inflammatory process, facilitate bacterial proliferation by increasing expression of vimentin, a protein to which Streptococcus adhere [2], and decrease the effectiveness of associated antibiotherapy.

Conclusion(s): Serious cases registered by the FPC, automated signal detection, and pharmacoepidemiological studies suggest that oral ibuprofen treatment for fever or pain during early stages of bacterial skin and soft tissue, or pleuropulmonary infections increases the risk of serious bacterial complications, when Streptococcus pyogenes or Streptococcus pneumonia are responsible. Experimental data refute the hypothesis that protopathic bias would be the only cause, and suggest implication of biological mechanisms, which has been sufficiently demonstrated to take measures of risk minimization.

\section{REFERENCE(S)}

1. Uppsala report 70. July 2015. https://www.who-umc.org/media/ 164373/ur70_web.pdf.

2. Bryant $A E$, Bayer $C R$, Aldape MJ, Stevens DL. The roles of injury and nonsteroidal anti-inflammatory drugs in the development and outcomes of severe group A streptococcal soft tissue infections: Current Opinion in Infectious Diseases. 2015;28(3):231-239. https://doi.org/10. 1097/QCO.0000000000000160

TABLE 1 Description of the 242 serious cases of infection under ibuprofen registered by the FPC

\begin{tabular}{|c|c|c|c|c|c|c|}
\hline & $\begin{array}{l}\text { Total } \\
\mathrm{n}\end{array}$ & $\begin{array}{l}\text { Children } \\
\text { n (\%) }\end{array}$ & $\begin{array}{l}\text { Duration of ibuprofen treatment } \\
\text { median [min-max] in days }\end{array}$ & $\begin{array}{l}\text { Antibiotherapy } \\
\mathrm{n}(\%)\end{array}$ & $\begin{array}{l}\text { Deaths } \\
\mathrm{n}(\%)\end{array}$ & $\begin{array}{l}\text { Sequelae } \\
\mathrm{n}(\%)\end{array}$ \\
\hline Acute community-acquired pneumonia & 113 & $49(44)$ & $4[1-14]$ & $39(35)$ & $4(5)$ & $4(4)$ \\
\hline Cellulitis & 106 & $63(60)$ & $3[1-21]$ & $17(16)$ & $3(3)$ & $7(7)$ \\
\hline Necrotising fasciitis & 23 & $13(57)$ & $3[2-8]$ & $0(0)$ & $4(18)$ & $9(41)$ \\
\hline
\end{tabular}


TAB LE 2 Description of the 13 pharmacoepidemiological studies reporting a measure of association between ibuprofen treatment and skin and soft tissue, or pleuropulmonary complications. OR: odds ratio; $\mathrm{Cl}$ : confidence interval; * NSAID including ibuprofen

\begin{tabular}{|c|c|c|c|c|}
\hline Reference & Country & Study design & Age of subjects & Measure of association \\
\hline \multicolumn{5}{|c|}{ Studies on skin and soft tissue complication } \\
\hline Choo and al. 1997 & United States & Nested case-control on database & Children & $\mathrm{OR}=3.1 ; 95 \% \mathrm{Cl}=0.1-19.7$ \\
\hline Zerr and al. 1999 & United States & Monocentric case-control on database & Children & $\mathrm{OR}=10.2 ; 95 \% \mathrm{Cl}=1.3-79.5$ \\
\hline Lesko and al. 2001 & United States & $\begin{array}{l}\text { Multicentric case-control in pediatrics } \\
\text { departments and outpatient }\end{array}$ & Children & $\mathrm{OR}=3.9 ; 95 \% \mathrm{Cl}=1.3-12.0$ \\
\hline Dubos and al. 2008 & France & $\begin{array}{l}\text { Nested case-control in pediatrics } \\
\text { department }\end{array}$ & Children & $\mathrm{OR}=4.8 ; 95 \% \mathrm{Cl}=1.6-14.4^{*}$ \\
\hline Mikaeloff and al. 2008 & United Kingdom & Nested case-control on database & $\begin{array}{l}\text { Children } \\
\text { Adults }\end{array}$ & $\begin{array}{l}\text { Rate ratio }=4.9 ; 95 \% \mathrm{Cl}=2.1-11.4 \\
\text { Rate ratio }=1.6 ; 95 \% \mathrm{Cl}=1.1-2.4^{*}\end{array}$ \\
\hline \multicolumn{5}{|c|}{ Studies on pleuropulmonary complication } \\
\hline Byington and al. 2002 & United States & Monocentric case-control on database & Children & $\mathrm{OR}=4.0 ; 95 \% \mathrm{Cl}=2.5-6.5$ \\
\hline François and al. 2010 & France & Multicentric case-control on database & Children & $\mathrm{OR}=2.6 ; 95 \% \mathrm{Cl}=1.5-4.4$ \\
\hline Voiriot and al. 2011 & France & $\begin{array}{l}\text { Monocentric cohort in intensive } \\
\text { care unit }\end{array}$ & Adults & $\mathrm{OR}=8.1 ; 95 \% \mathrm{Cl}=2.3-28.0 *$ \\
\hline Messika and al. 2014 & France & Monocentric cohort on database & Adults & $\mathrm{OR}=4.0 ; 95 \% \mathrm{Cl}=1.1-15.4^{*}$ \\
\hline Elemraid and al. 2015 & United Kingdom & $\begin{array}{l}\text { Nested case-control in pediatrics } \\
\text { department }\end{array}$ & Children & $\begin{array}{l}\text { OR }=1.9 ; 97.5 \% \text { credible } \\
\text { interval }=0.8-3.2\end{array}$ \\
\hline $\begin{array}{l}\text { Le Bourgeois } \\
\text { and al. } 2016\end{array}$ & France & $\begin{array}{l}\text { Multicentric case-control in pediatrics } \\
\text { departments and outpatient }\end{array}$ & Children & $\mathrm{OR}=2.8 ; 95 \% \mathrm{Cl}=1.4-5.6$ \\
\hline Basille and al. 2017 & France & Nested case-control in hospital & Adults & $\mathrm{OR}=2.6 ; 95 \% \mathrm{Cl}=1.0-6.6$ \\
\hline Krenke and al. 2018 & Poland & $\begin{array}{l}\text { Nested case-control pediatrics } \\
\text { department }\end{array}$ & Children & $\mathrm{OR}=5.1 ; 95 \% \mathrm{Cl}=1.5-17.4$ \\
\hline
\end{tabular}

\section{Oc047 | An adverse event risk prediction model using a weighted polypharmacy count in rheumatoid arthritis}

\author{
Miriam Dixon-Zegeye ${ }^{1}$; Katie Bechman ${ }^{2}$; Andrew Cope ${ }^{2}$; \\ James Galloway ${ }^{2}$ \\ ${ }^{1}$ University College London; ${ }^{2}$ King's College London
}

Introduction/Background \& Aims: Multi-morbidity is highly prevalent in contemporary rheumatoid arthritis populations. The ability to accurately risk stratify patients would be advantageous when making treatment decisions, particularly the prescription of immunomodulatory drugs which are known to increase the risk of adverse events. Medication counts are routinely collected in electronic health records. We have previously shown that medication count predicts adverse events and may provide more information than established comorbidity indices, e.g., Rheumatic Disease Comorbidity Index (RDCI). This study set out to evaluate the potential of a weighted medication count for predicting serious adverse events.

Method/Summary of work: We analysed data from the British Society for Rheumatology Biologics Register (BSRBR-RA), a prospective observational cohort study. The population included adult patients with an established diagnosis of rheumatoid arthritis (RA). Patients were selected for study involvement because they were commencing either a new conventional or biologic disease-modifying antirheumatic drug (DMARDs). Medication count, excluding DMARDs, was recorded at baseline, and medications were categorised into classes according to indication and mode of action. The primary outcome was serious adverse event by individual class. This was estimated using a single failure Cox proportional hazards model. Hazard rates were used to compute weighted medication counts. The weighted and unweighted medication count models were evaluated by comparing Akaike Information Criterion (AIC), Bayesian Information Criterion (BIC) and Harrel's C coefficients.

Results/Discussion: The analysis included 17,826 patients. The demographics of the cohort represented a typical RA population $(76 \%$ female, mean age 57 (SD 12)). 12,547 patients experienced at least one adverse event during follow up for 3 years, with an event rate 23.7 per 100 years (95\% Cl 23.2-24.1). In the unweighted medication count model, the hazard ratio for serious adverse event was 1.10 (95\% $\mathrm{Cl} 1.09-11)$. For anticoagulation, the unadjusted hazard ratio for serious adverse event was 1.97 (95\% Cl1.87-2.07). For musculoskeletal medications, the unadjusted hazard ratio was $0.81(95 \% \mathrm{Cl}$ 0.78-0.85). The weighted model included two points for anticoagulation and one point per medication class with the exception of musculoskeletal medications which scored zero. The weighted model performed better at predicting serious adverse events when compared to unweighted medication counts; $\mathrm{AIC}=186990$ vs 231421, $\mathrm{BIC}=187000$ vs 231431, and Harrell's C 0.59 (95\% Cl 0.58-0.59) vs $0.58(0.58-0.59)$ (Table 1). 
TABLE 1 Evaluation of weighted and unweighted medication count models using AIC, BIC and Harrell's C

\begin{tabular}{|c|c|c|c|c|c|c|c|c|}
\hline & \multicolumn{4}{|c|}{ Weighted polypharmacy model } & \multicolumn{4}{|l|}{ Medication count } \\
\hline Unadjusted & $1.26(1.25-1.28)$ & $186990^{\mathrm{a}}$ & $187000^{b}$ & $0.59(0.58-0.59)$ & $1.13(1.12-1.13)$ & $231421^{a}$ & $231431^{b}$ & $0.58(0.58-0.59)$ \\
\hline
\end{tabular}

Abbreviations: AIC, Akaike's information criterion; BIC, Bayesian information criterion (BIC).

${ }^{a}$ Best fit, lower Akaike's information criterion (AIC) value demonstrates better model fit.

${ }^{b}$ Best fit, lower Bayesian information criterion (BIC) value demonstrates better model fit.

Conclusion(s): The weighted medication count provides an improved model for predicting serious adverse events compared to a raw medication count. This work builds on a previous study by Bechman et al (1) demonstrating that medication count predicts adverse events. This weighted medication count could be used to help clinicians risk stratify patients when making treatment decisions.

\section{REFERENCE(S)}

1. Katie Bechman, Benjamin D Clarke, Andrew I Rutherford, Mark Yates, Elena Nikiphorou, Mariam Molokhia, Sam Norton, Andrew P Cope, Kimme L Hyrich, James B Galloway, Polypharmacy is associated with treatment response and serious adverse events: results from the British Society for Rheumatology Biologics Register for Rheumatoid Arthritis, Rheumatology, https://doi.org/10.1093/rheumatology/kez037

\section{Mixed Oral Communications 1}

\section{OC048 | MicroRNA-126 is a marker of vascular dysfunction in chronic kidney disease}

\author{
Kathleen Scullion; Bastiaan Vliegenthart; Tariq Farrah; \\ Neeraj (Bean) Dhaun; James Dear \\ The University of Edinburgh
}

Introduction/Background \& Aims: Chronic kidney disease (CKD) affects millions of people worldwide and the incidence is growing exponentially with the disease now being responsible for over 25 million deaths per year. There is a strong correlation between people suffering from CKD and the development of cardiovascular disease (CVD). CKD patients with stage 5 of the condition are five times more likely to develop CVD than the general population. There is an unmet clinical need for biomarkers that specifically report vascular health in CKD. microRNAs (miRs) are a class of RNA molecules that are 20 to 25 nucleotide long noncoding strands, involved in translational repression and cleavage. They can exhibit organ specificity, making them a significant emerging class of biomarker for many diseases and can be used in a pre-clinical setting and translated for use in patients. MiR-126 is endothelial cell-enriched and present throughout the vasculature. We examined the relationship between miR-126 and chronic vascular dysfunction in patients with CKD and maintenance haemodialysis dependent end-stage renal disease (ESRD).

Method/Summary of work: Blood samples were collected from 60 healthy subjects, 30 patients with CKD and 15 patients with ESRD on haemodialysis. Samples from ESRD patients were collected immediately pre- and post-haemodialysis. The concentration of a panel of circulating microRNAs were measured by qRTPCR and absolute quantification was performed using a standard curve. Results were correlated with clinical parameters currently used to assess vascular function. Statistical significance was determined by Mann-Whitney $U$ test or Wilcoxon matched-pairs signedrank test. The study was approved by the local research ethics committee and performed in accordance with the Declaration of Helsinki.

Results/Discussion: Circulating miR-126 was substantially reduced in ESRD patients pre-haemodialysis compared with healthy volunteers (Figure 1); pre-haemodialysis median 0.20fM (IQR 0.02-1.35fM), healthy volunteer median 70.41fM (IQR 17.21-770.00fM), $P<0.0001$. In patients with CKD, the concentration of miR-126 was significantly higher than pre-dialysis, median $71.75 \mathrm{fM}$ (IQR 30.39-162.4fM), $P<0.0001$. Haemodialysis increased miR-126 to a median value of $0.93 \mathrm{fM}$ (IQR 0.31-4.68), $P=0.0002$. After haemodialysis, patients still had a lower concentration of circulating miR-126 than in health and CKD $(P<0.0001)$. miR-126 also correlated with current gold standard measurements for vascular function, including pulse wave velocity.

Conclusion(s): miR-126 is substantially lower in ESRD compared to healthy volunteers and patients with CKD. Circulating miR-126 increases with haemodialysis. These data suggest that miR-126 has a potential role as a biomarker of vascular health and could track the progression from CKD to ESRD once a defined range for miR-126 has been further established. 


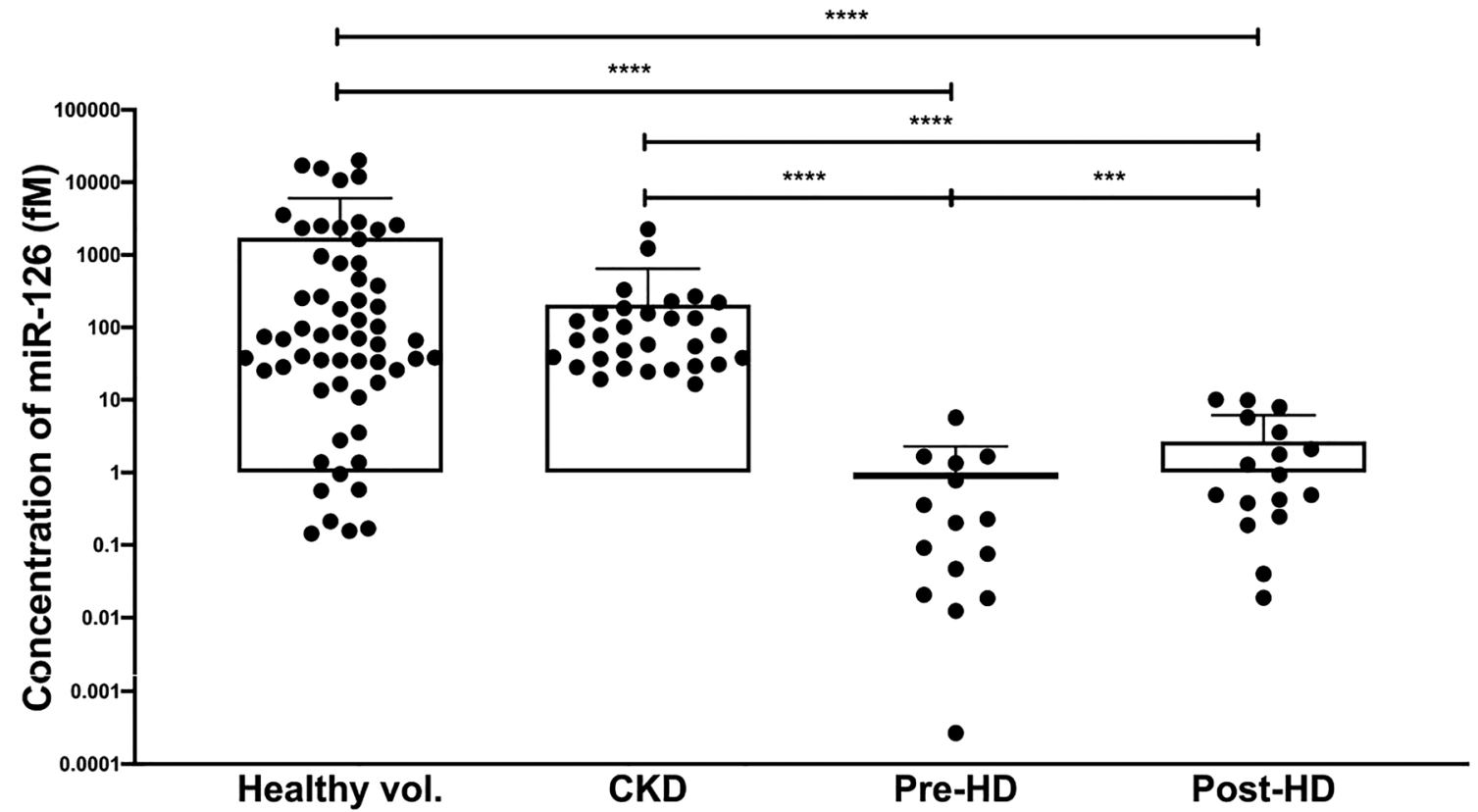

FIGURE 1

\section{OC049 | Effects of a PAM antibody to the insulin receptor on metabolic homeostasis are dependent on insulin sensitivity}

Barbara Musial; Asha Seth; Alessandra Rossi; James Dodgson; Anna Sigurdardottir; Bojana Popovic

AstraZeneca

Introduction/Background \& Aims: Chronic hyperinsulinaemia is a key contributing driver in the initiation of type 2 diabetes (T2D). Therapies designed to enhance insulin signalling may therefore represent effective T2D treatments. Here, we use positive allosteric modulation (PAM) of the insulin receptor (InsR) to increase the drive through InsR and assess how enhancement of insulin signalling impacts on disease progression in murine models of diabetes.

Method/Summary of work: Several in vitro assays were used to verify mechanism of action of IRM0010 comparing with the isotype control (IgG), (Table 1). The effect of IRM0010 was also tested in streptozotocin (STZ)/high-fat diet (HFD) and insulin resistant/obese $d b / d b$ models, using IP administration. For the STZ/HFD model, 4-week treatment, mice were randomly allocated for: non-STZ-lgG,

TABLE 1 In vitro experiments demonstrating PAM activity of monoclonal antibody IRM0010.

\begin{tabular}{|c|c|c|c|c|}
\hline Type of assay & Method & Readout & Cell type & Results \\
\hline Receptor binding & $\begin{array}{l}\text { Cells were exposed to labelled } \\
\text { human } 10 \mathrm{nM} \text { insulin in the } \\
\text { presence of } 50 \mathrm{nM} \\
\text { IRM0010/lgG }\end{array}$ & $\begin{array}{l}\text { Real-time affinity using } \\
\text { Ligand Tracer }\end{array}$ & U2OS hIRb cells $(n=3)$ & $\begin{array}{l}\text { IRM0010 increased insulin binding } \\
\text { affinity }\left(K_{D}\right) \text { by } 5 \text {-fold, } K_{D}=5.1 \\
+/-1.7 \text { versus } K_{D}=1.1+/-1.1 \\
\quad+/-0.2\end{array}$ \\
\hline Insulin retention & $\begin{array}{l}\text { Cells incubated in presence of } \\
\text { titrated IRM0010/IgG and } \\
250 \mathrm{nM} \text { insulin-AF488 for } \\
30 \mathrm{~min} .\end{array}$ & $\begin{array}{l}\text { Insulin-AF488 intensity } \\
\text { within total cell }\end{array}$ & U2OS hIRb cells $(n=3)$ & $\begin{array}{l}\text { Insulin-AF488 retained after } 1 \text { hour } \\
\text { only in presence of IRM0010 } \\
\text { (EC50 }=7.2+/-3.0 \mathrm{nM}) \text {, but not } \\
\text { in presence of IgG } \\
\text { (EC50 > 400nM) }\end{array}$ \\
\hline \multirow[t]{3}{*}{ Cellular signalling } & $\begin{array}{l}\text { Cells incubated in presence of } \\
1 \mu \mathrm{M} \text { IRM0010/lgG and } \\
\text { 100nM insulin for } 30 \mathrm{~min}\end{array}$ & $\begin{array}{l}\text { Phosphorylation of } \\
\text { Ser473 Akt }\end{array}$ & $\begin{array}{l}\text { Human insulin sensitive } \\
\text { hepatocytes }(n=3)\end{array}$ & $\begin{array}{l}\text { Activation of Akt increased by } 25 \% \\
\qquad(p<0.0001)\end{array}$ \\
\hline & & & $\begin{array}{l}\text { Mouse insulin sensitive } \\
\text { hepatocytes }(n=3)\end{array}$ & $\begin{array}{l}\text { Activation of Akt increased by } 34 \% \\
\qquad(p<0.0001)\end{array}$ \\
\hline & $\begin{array}{l}\text { Cells incubated with } 141 \mathrm{nM} \\
\text { insulin for } 20 \mathrm{~h} \text { prior to } \\
\text { incubation in presence of } \\
1 \mu \mathrm{M} \text { IRM0010/lgG and } \\
100 \mathrm{nM} \text { insulin for } 30 \mathrm{~min}\end{array}$ & & $\begin{array}{l}\text { Mouse insulin resistant } \\
\text { hepatocytes }(n=3)\end{array}$ & $\begin{array}{l}\text { Activation of Akt increased by } 24 \% \\
\qquad(p<0.0001)\end{array}$ \\
\hline
\end{tabular}


STZ-IgG and STZ-IRM0010, $\mathrm{n}=12$, each group. All mice were then treated twice weekly by $10 \mathrm{mg} / \mathrm{kg} /$ day of IRM0010/lgG. A GTT at $1 \mathrm{~g} / \mathrm{kg}$ and ITT at $0.75 \mathrm{IU} / \mathrm{kg}$ were performed at 3rd and 4th week, respectively. For the $d b / d b$ model, 5-week treatment was completed in: $d b /+-\lg G, d b / d b$-IgG and $d b / d b$-IRM0010, $n=12$, each group. Mice were treated twice weekly of IRM0010/lgG (dose from $10 \mathrm{mg} / \mathrm{kg} /$ day to $200 \mathrm{mg} / \mathrm{kg} /$ day). An ITT, $3 \mathrm{IU} / \mathrm{kg}$ was performed in the 5 th week of treatment. At final day, mice were sub-allocated for an insulin/PBS stimulation $5 \mathrm{lU} / \mathrm{kg}(\mathrm{n}=5 / \mathrm{n}=3$ ) to measure activation of Akt in selected tissues and plasma HbA1c levels $(n=2-4)$. Fed and fasted glucose, fasted insulin, and biometry were evaluated weekly in both models.

Results/Discussion: The IRM0010 monoclonal antibody demonstrated PAM activity in binding, imaging, and cellular signalling assays (Table 1). IRM0010 treatment in the STZ/HFD model lowered fasting blood glucose $(p<0001)$, lowered fasting plasma insulin $(p<0.001)$, normalised the response to a glucose challenge and improved insulin sensitivity $(p<0.0001)$. There was a modest effect of IRM0010 on fed glucose levels which was significant at $2(p<0.01)$ and 4 weeks $(p<0.05)$. No effect of IRM0010 was observed on body weight. In contrast, in the $d b / d b$ model, fed glucose levels $(p<0.01)$ and terminal HbA1c $(p<0.05)$ were significantly elevated. Weight gain was dramatically reduced $(p<0.0001)$ but worsened insulin sensitivity ( $p<0.01$ ) by IRM0010 treatment. In addition, fasted insulin levels were increased $(p<0.01)$ and insulin stimulation showed a reduced level of pAkt in muscle $(p=0.004)$, adipose $(p<0.03)$, and liver $(p<0.04)$.

Conclusion(s): IRM0010 is an effective positive modulator of InsR in vitro and improved glycaemic control in STZ/HFD model. However, in the obese $d b / d b$ model, insulin sensitivity was worsened. These studies suggest that IRM approaches may better serve patients with in T1D or in insulinopaenic states, where enhanced InsR function may improve a low insulin signalling drive. This intervention in hyperinsulinaemic states could exacerbate the disease likely though further insulin induced insulin resistance.

\section{OC051 | Dynamic chemical labelling} accurately quantifies microRNA-122 and reveals the presence of isomiRs that compromise PCR analysis in drug-induced liver injury

\author{
James Dear ${ }^{1}$; Barbara López-Longarela ${ }^{2}$; Emma Morrison ${ }^{3}$; \\ John Tranter ${ }^{3}$; David Duffy ${ }^{4}$; Juan Díaz-Mochón ${ }^{2}$ \\ ${ }^{1}$ The University of Edinburgh; ${ }^{2}$ Destina Genomics; ${ }^{3}$ University of \\ Edinburgh; ${ }^{4}$ Quanterix Corporation
}

Introduction/Background \& Aims: MicroRNAs (miRs) are promising biomarkers reported to be stable in blood. miR-122 is an hepatocyte specific miR that is a biomarker for drug-induced liver injury (DILI). For adoption of miR-122 into clinical practice there are fundamental analytical challenges to overcome. These include preventing sample degradation, and the development of assays suitable for clinical use. Our objective was to develop an extraction- and amplification-free detection platform for miR-122 that addresses these challenges and has translational utility in context of DILI.

Method/Summary of work: We have developed a PCR-free, sensitive and specific assay for measuring miR-122. This dynamic chemical labelling (DCL) assay is based on miR-122 hybridization to an abasic peptide nucleic acid probe attached to superparamagnetic beads, followed by the specific incorporation of a labelled nucleobase. Labels are detected using single molecule arrays. Analytical performance was quantified using synthetic miR-122, human samples from patients with paracetamol overdose $(\mathrm{N}=192)$, a pre-clinical rat model $(\mathrm{N}=12)$ and dogs with biopsy-proven liver disease $(N=12)$. DCL assay, PCR and small RNA sequencing were used to investigate serum miR-122 degradation.

Results/Discussion: The single molecule DCL assay specifically measured miR-122 directly from $10 \mu \mathrm{L}$ of serum without any extraction steps, with a fit-for-purpose limit of detection of $1.32 \mathrm{pM}$. In 192 human serum samples, DCL accurately identified patients at risk of DILI (area under ROC curve 0.98 (95\%Cl 0.96-1), $P<0.0001)$. The miR-122 assay also quantified liver injury in rats and dogs. When $D C L$ beads were added to serum, the miR-122 signal was stabilised (no loss of signal after 14 days at room temperature). By contrast, there was substantial degradation of miR-122 in the absence of beads $(\approx 60 \%$ lost in 1 day). RNA sequencing demonstrated the presence of multiple miR-122 isomiRs with DILI that were at low concentration or not present in healthy patient serum. Sample degradation over time produced more isomiRs, particularly rapidly with DILI. PCR was inaccurate when analysing miR-122 isomiRs, whereas the DCL assay demonstrated accurate quantification.

Conclusion(s): The DCL assay can accurately measure miR-122 directly from serum and plasma to diagnose liver injury in humans and other species, and can overcome important microRNA biomarker analytical and biological challenges.

\section{Posters, Tuesday 17th December, 13:15}

Poster Session: Education and Skills

\section{P258 | Is low concentration of tranexamic acid related to intraoperative blood loss in scoliosis surgery?}

Paula Alexandra Sa ${ }^{1}$; Maria Conceição Manso $^{2}$; Luisa Barreiros ${ }^{3}$; Marcela A. Segundo ${ }^{3}$; Sibylle Kietaibl ${ }^{4}$

${ }^{1}$ CHUP; ${ }^{2}$ Faculty of Health Sciences, University Fernando Pessoa; ${ }^{3}$ LAQV, REQUIMTE, Department of Chemical Sciences, Faculty of Pharmacy, University of Porto; ${ }^{4}$ Department of Anaesthesia and Intensive Care, 
Evangelical Hospital Vienna and Sigmund Freud Private University, Vienna

Introduction/Background \& Aims: Tranexamic acid (TXA) is recommended in major orthopedic surgery as an antifibrinolytic drug to prevent and to treat hyperfibrinolysis (1). However, dosing regimen vary widely and the optimal dose has not yet been established. Also the relationship between the dose and plasma concentration is unclear. We aimed to measure plasma levels of TXA in patients receiving TXA during scoliosis surgery and their clinical response.

Method/Summary of work: This study was performed as a prospective observational study after Ethics Committee Approval and informed consent. Forty-two patients undergoing scoliosis surgery received TXA loading dose of $10 \mathrm{mg} / \mathrm{kg}$ over 15 minutes, followed by continuous intravenous infusion at $1 \mathrm{mg} / \mathrm{kg} / \mathrm{h}$ from the beginning of the surgery until skin closure. Blood samples were drawn 30 minutes after the bolus, before transfusion of red blood cells, at the end of surgery. We measured plasma concentrations of TXA using liquid chromatography coupled to triple quadruple-tandem mass spectrometry (2). We assessed the in vitro-threshold needed for inhibiting standardized fibrinolysis at 10-15 $\mu \mathrm{g} / \mathrm{ml}$ TXA (3). Intraoperative estimated blood loss (IOEBL) was determined by measuring the suction drainage minus any irrigation fluid and weighing the sponges. IOEBL was reported per estimated blood volume. Quantitative variables were described using the median values and corresponding 25th and 75th percentiles and the range. Correlation between TXA plasma levels and IOEBL were calculated using Spearman coefficient correlation with IBM $^{\circledR}$ SPSS $^{\circledR}$ Statistics vs. $25(\alpha=0.05)$.

Results/Discussion: TXA plasma concentrations were above $10 \mu \mathrm{g} / \mathrm{ml}$ in 35 patients and below in 7 patients at the end of surgery. Median (IQR;[range]) plasma concentration was 12.7 (10.5-14.5; [5.7-19.1]) $\mu \mathrm{g} / \mathrm{ml}$. IOEBL was 44.7 (40.3-67.5; [21.2-73.99]) \% at the end of surgery. There was a significant moderate inverse correlation between IOEBL and TXA plasma concentration $(r=-0.554$; $P<0.001)$.

Conclusion(s): This study is the first to report TXA plasma levels in scoliosis surgery. Despite a standardized infusion protocol TXA plasma concentrations above $10 \mu \mathrm{g} / \mathrm{ml}$ were observed in only $83 \%$ of patients. Low concentrations were associated with increased IOEBL.

\section{REFERENCE(S)}

1. Kozek-Langenecker SA et al. Management of severe perioperative bleeding. Eur J Anaesthesiol 2017; 34(6):332-395.

2. Barreiros $L$ et al. Validation of a straightforward strategy based on UHPLC coupled with tandem mass spectrometry for quantification of tranexamic acid in human plasma. Microchemical J 2019;144:144-150

3. Picetti $R$ et al. What concentration of tranexamic acid is needed to inhibit fibrinolysis? A systematic review of pharmacodynamics studies. Blood Coagulation and Fibrinolysis 2019;30:1-10

\section{P262 | Does obligatory clinical trial registration impact quality of trial protocols? A comparison of global and local regulatory trials}

\author{
Ashish Kakkar ${ }^{1}$; Biswa Mohan Padhy²; Sudhir Chandra Sarangi ${ }^{2}$; \\ Yogendra Kumar Gupta ${ }^{2}$ \\ ${ }^{1}$ Postgraduate Institute of Medical Education and Research; ${ }^{2}$ All India \\ Institute of Medical Sciences Bhubaneswar
}

Introduction/Background \& Aims: Drug research and development is becoming increasingly complex, risky, lengthy and expensive proposition. This has led to globalization of clinical trials to meet the two objectives of saving costs and time. Several studies in the past have found significant positive impact of clinical trial registration on the reporting quality of randomized trials. However, the impact of trial registration on the quality of clinical trial protocols remains unknown. Therefore, the present study was planned to assess the influence of obligatory trial registration introduced in India on the methodological quality of regulatory protocols and to compare the impact between global and local clinical studies.

Method/Summary of work: We conducted a retrospective cohort study of trial applications submitted to the drug regulator authority for initial review in the periods immediately preceding and succeeding the introduction of obligatory trial registration in the country, i.e., 15th June 2009 (1). The two time periods chosen were from January 2007 to May 2009 (P1) and from July 2009 to December 2011(P2). A total of 150 trial protocols - seventy-five from each time period were randomly selected and subject to analysis. A set of twelve key methodological quality characteristics were chosen to evaluate the selected study protocols. These were drawn from validated and widely accepted tools, checklists, and guidelines and included clearly defined eligibility criteria and study outcomes, randomization, blinding, allocation concealment, presence of control group, description of statistical analysis plan, sample size calculations, assessment of compliance, and handling of withdrawals among others (2-4).

Results/Discussion: Majority of the submitted protocols were for the development of new chemical entities (81.3\% in P1 vs 74.7 in P2). Nearly $59 \%$ of the study protocols in P1 were for global clinical trials as compared to $67 \%$ in P2. There were no significant differences in the baseline characteristics of trial applications in the two study periods except for the phases of clinical trials. Comparing the two study periods, proportion of blinded studies increased from $44 \%$ to $61 \%(p=0.03)$, description of allocation concealment more than doubled in $\mathrm{P} 2$ vs. $\mathrm{P} 1$ ( $p=0.001$ ), description of clearly defined study outcomes improved from $41 \%$ to $80 \%(p<0.001)$, and justification of sample size increased from $38 \%$ to nearly $70 \%(p<0.001)$. Significant improvements were also recorded for handling of subject withdrawals and description of statistical analysis plans. While the mean cumulative scores for the global clinical trials improved significantly from 
$7.5 \pm 0.37$ in $\mathrm{P} 1$ to $10.2 \pm 0.28(p<0.0001)$, no significant improvements were noted for local clinical trials.

Conclusion(s): We found significant improvements in the methodological quality of trial protocols especially for features related to minimization of bias and statistical procedures, which could be attributed to obligatory trial registration. Overall, the noted improvements were limited to global clinical trials with further room for improvement in other remaining key quality characteristics.

\section{REFERENCE(S)}

1. Clinical Trial Registry - India [Internet]. Available from: http://ctri.nic. in/Clinicaltrials/login.php

2. Moher D, Hopewell S, Schulz KF, Montori V, Gøtzsche PC, Devereaux PJ, et al. CONSORT 2010 Explanation and Elaboration: updated guidelines for reporting parallel group randomised trials. BMJ. 2010 Mar 24;340:c869. (DOI: https://doi.org/10.1136/bmj.c869)

3. Statistical principles for clinical trials [Internet]. Available from: http:// www.emea.europa.eu/docs/en_GB/document_library/Scientific_ guideline/2009/09/WC500002928.pdf.

4. Jadad AR, Moore RA, Carroll D, Jenkinson C, Reynolds DJ, Gavaghan DJ, et al.. Assessing the quality of reports of randomized clinical trials: is blinding necessary? Control Clin Trials. 1996;17(1):1-12.

\section{P263 | Inherited Blindness Summer School, University College Dublin 2019}

Ailis Moran; Rebecca Ward; Breandán Kennedy

University College Dublin

Introduction/Background \& Aims: The goals of the Summer School were to promote Pharmacology, raise awareness of inherited vision loss and endorsing scientific careers for second level students. The course provided a platform for students to engage with University life. Students were exposed to hands-on laboratory experience which effectively introduced scientific enquiry and critical thinking. A practical-based method of teaching science subjects was a unique experience, one not readily available at second level in Ireland [1].

Method/Summary of work: The event took place over four days with involvement from clinicians, researchers, patients and supporting charities. Secondary schools throughout Ireland were contacted via email, inviting senior cycle students to register. A total of 22 students from 5 different counties across Ireland completed the course. There was gender balance with $40.9 \%$ males and $59.1 \%$ females.

The course introduced students to Pharmacological research in a University setting. Students engaged in afternoon laboratory sessions conducting experiments based on topics discussed in the morning lectures. A logical workflow was integrated into each of the laboratory sessions and stimulated students to apply their knowledge in a practical situation. Practicals involved identifying visually impaired zebrafish using visual behavioural assays and PCR genotyping. Drug action was also undertaken using a drug to restore vision in the blind zebrafish model.
The summer school involved a group media project, requiring students to effectively communicate scientific knowledge learned throughout the week to a lay audience, highlighting the importance of scientific dissemination. Presentations included theatrical performances, interactive quizzes and rap medleys.

The impact of the summer school was measured through an anonymous questionnaire given to the students which gathered information on their experience. The results highlighted the popularity of the practical sessions. To conduct this survey and disseminate results, approval was granted from the Human Research Ethics Committee in UCD.

Results/Discussion: The feedback was highly positive. In particular, the laboratory sessions were thoroughly successful, emphasising the need for a combination of practical and theory-based learning. The blend of teaching methods in a problem-based learning approach, instilled critical thinking and team work amongst the students [2].

Conclusion(s): The course allowed students to experience research first hand in an academic setting. The feedback indicates the workshop is an excellent educational tool for introducing pharmacology and disease.

\section{REFERENCE(S)}

1. A. Walshe, "From curriculum to classroom in upper second level science," PhD thesis, Dublin City University, 2015.

2. S. Loyens, "Problem-based learning," Application to learning and teaching, vol. 3, pp. 403-425, 2011.

\section{P264 | Innovations in attracting ages 9-18 to careers like pharmacology}

\section{Yewande Okunoren-Oyekenu}

California Intercontinental University

Introduction/Background \& Aims: Problems exist with choosing careers like pharmacology due to lack of information on career choices. Majority of students think that science disciplines are quite difficult or have no idea what to study [1]. This project is aimed at improving students' interest in science subjects, especially pharmacology.

Method/Summary of work: Six scientists took part in a competition called; I'm a scientist get me out of here, that mimics reality TV shows like "I am a celebrity get me out of here." It was organized for school students to ask real-life scientists questions as well as have the opportunity to chat online with them in the presence of their teachers or parents depending on if they were at school or home respectively. They were then asked to vote for their favorite scientist based on performance in answering questions and their interest in the scientist's discipline. 461 students from 10 schools in the UK with age group 9-18 took part in the 2-week program. Scientist 1 happened to be a pharmacologist with specialization in neuroscience. Scientist 1 came second based on the students' votes and interest in the field while 


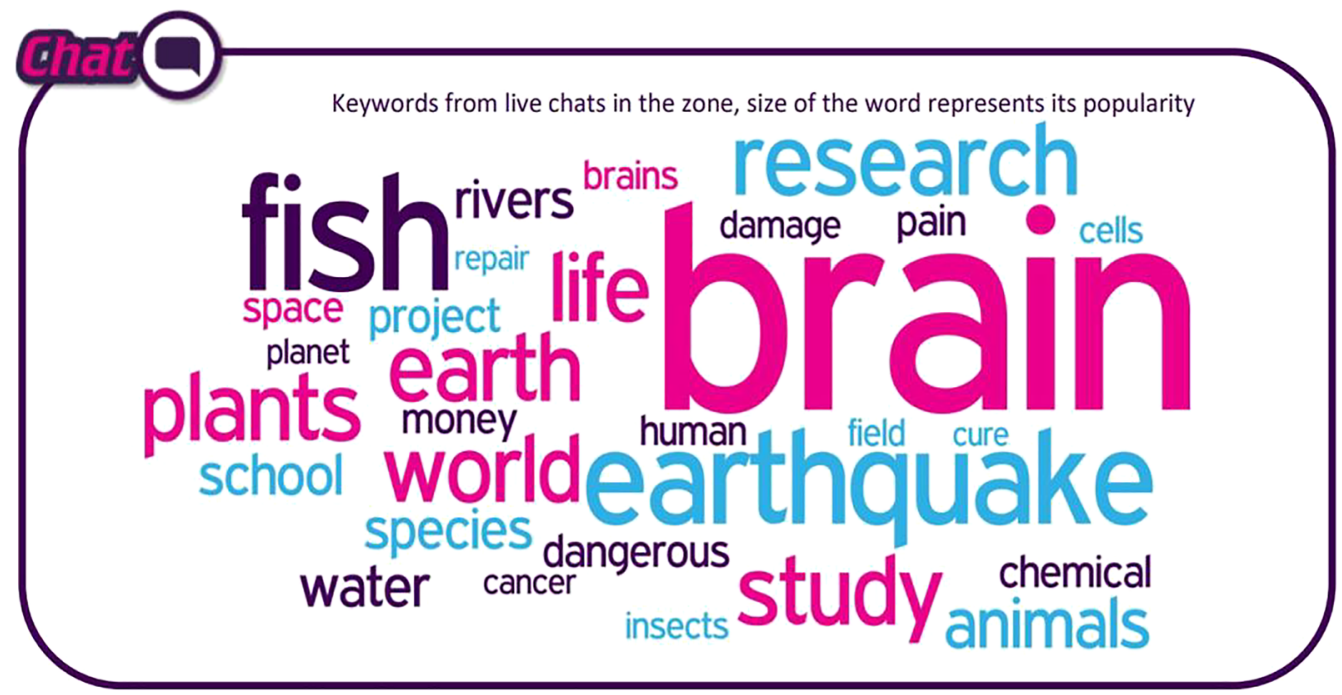

FIGURE 1. Words that came up during the career talk. Size of the word shows its popularity

scientist 6 came first and won the prize of $£ 500$. Scientists also voted for their best students for them to have a chance at the prize of $£ 250$.

1. Researching the use of nitric oxide as an anaesthetic and the effect the gas has on brain cells

2. Studying earthquakes and how they behave, the dangers they pose and the opportunities offered

3. Technician in the Biomedical Science department at the University of Sheffield

4. PhD student using mathematics to model Wi-Fi signals

5. Manager at a chemical plant responsible for the quality and productivity the plant delivers

6. Studying the impact of non-native plants on small rivers and streams

Results/Discussion: As seen in Figure 1, the students were more interested in questions relating to brain, earthquake, fish, research, etc. It is possible that they had no idea about neuropharmacology and neonatal study before the competition hence the reason they asked various questions in these areas. This was the same for earthquake and aquatic study.

Conclusion(s): The competition had a great impact on students' knowledge about pharmacology and the project will continue to seek the help of pharmacologist, psychologist, and other scientists in rare disciplines.

\section{REFERENCE(S)}

1. I'm a scientist get me out of here (2018). Berkelium zone report. Retrieved from: https://about.imascientist.org.uk/2018/berkeliumzone-report-june-2018/

\section{P265 | Characteristics of biomedical student researchers and factors influencing their research output}

Igor Radanovic ${ }^{1}$; Kristian Dominik Rudez ${ }^{2}$; Deni Rkman²;

Robert Likic ${ }^{3}$; Jeroen van Smeden ${ }^{1}$

${ }^{1}$ Centre for Human Drug Research, Leiden; ${ }^{2}$ School of Medicine, University of Zagreb; ${ }^{3}$ University Hospital Centre Zagreb, Department of Internal Medicine, Division of Clinical Pharmacology and Therapeutics;

School of Medicine, University of Zagreb

Introduction/Background \& Aims: Scientific publications and research are the cornerstone of today's evidence-based medicine, so it is important for biomedical students to get sufficient experience at an early stage during their studies. Although there is a continuing effort to introduce students to research opportunities, generally a minority of them still manage to publish successfully. To better understand this issue, both positive and negative influencing factors should be identified. The aim of this study was to assess the characteristics of students who participate in research activities and to determine possible influencing factors for research output.

Method/Summary of work: A survey was conducted using an onlinebased questionnaire from April to June 2019 among different groups of students of the biomedical field. The questionnaire consisted of multiple-choice, open-choice and Likert score questions and tested several different categories, such as basic demographic information, quantity and quality of publications, research motivation, extracurricular activities and future plans.

Results/Discussion: The total number of responses was 230 (response rate of $74 \%$ ). Almost two thirds of the participants (64\%) were female and $35 \%$ were male. Out of all students, 106 (46\%) indicated they were involved in research in some way (either participating in projects, conferences or publications). Only 27 (12\%) students reported to have 
a journal publication while providing actual journal details. Additionally, $78 \%$ of students had never presented and $74 \%$ did never publish an abstract at a conference. The average grade for all passed courses of all surveyed students was 4.21 (out of a possible 5.00) and there was no statistically significant difference between those who have published a journal article and those who have not $(p>0.5$, Student $t$ test). Poor guidance was identified as the greatest hurdle students faced in trying to conduct research (39\%), followed by time constraints (26\%) and lacking teacher support (13\%). Most responders found better employment options and staying updated on developments in their field of interest as most important motives for doing research.

Conclusion(s): In general, there are still barriers that need to be overcome for students to engage more in research opportunities. In order to increase the number of students who publish, it is necessary to promote student abstract submission and scientific conference attendance. Specifically, more support from the administration and more guidance by mentors should be present in order to boost student research interest as well as output.

\section{P266 | Bug Off Pain®: An educational virtual reality game in spider venoms and chronic pain for public engagement}

Lucka Bibic $^{1}$; Justinas Druskis ${ }^{2}$; Sam Walpole ${ }^{1}$; Jesus Angulo ${ }^{1}$; Leanne Stokes ${ }^{1}$

${ }^{1}$ University of East Anglia; ${ }^{2}$ AKKA

Introduction/Background \& Aims: Although the virtual reality (VR) games have been capturing the public imagination for decades, harnessing this technology to benefit education and engagement in pharmacologically-relevant subjects, hasn't been extensively pursued. Here, we report a development and implementation of a multiplatform, immersive, engaging and educational VR game called Bug Off Pain that may assist general public and high school students in their understanding of the biochemistry and pharmacology behind the venoms in relation to chronic pain [1].

Method/Summary of work: The evaluation of general public opinions was performed through 14 statements with responses based on a 10-point Likert-type scale. The evaluation of VR-based learning was conducted by a cohort of 44 high school students (ages 17-18). By using pre- and post-tests, together with the multiple comparison test (ANOVA, Wilcoxon test) between the relative differences of the average number of correct answers (ANCA), the comparison between the ANCA in the experimental and control group of students was carried out.

Results/Discussion: We show that the game is fun, dynamic, easy to play, has an attractive design, easy to understand interface, relevant content, and that extending Bug Off Pain to other science-relevant subjects might be educationally beneficial as well. Our data also demonstrate the effectiveness of the instructional role of the game in promoting learning about chronic pain and the biochemistry of venom via a VR medium. Possible reasons for its valuable educational benefits include not only correctly using critical thinking skills and problem-solving abilities, but also framing individual creativity and selfdirection-all of which stand out as the less-tangible, non-academic benefits.

Conclusion(s): Together, our findings suggest that VR games, such as Bug Off Pain, allow the traditional public engagement process to become more appealing and educationally effective to the general public and students when permeated with virtual reality tools. Moreover, our results have a clear implication on how to design and implement virtual reality games in science education and offer insights into the implications for the broader teaching practice.

\section{REFERENCE(S)}

1. Bibic L, Druskis J, Walpole S, Angulo J, Stokes L (2019) Bug Off Pain: An educational virtual reality game in spider venoms and chronic pain for public engagement. J Chem Educ. 7: 1486

\section{P267 | Evaluation of the academic tutoring system for MPharm with Foundation students: What can we change to provide effective academic support?}

Silvia Amadesi

School of Pharmacy, Pharmacology Division, University of Reading

Introduction/Background \& Aims: Most UK universities operate a personal/academic tutoring system (ATS) (1) aimed to provide students with holistic guidance on an academic and personal level. An ATS is critical for student engagement particularly during the first year at university (2). It also promotes connection (3) and a sense of belonging to the academic community (4) and increases retention (2). Our student records indicated that only a third of our Year1 MPharm with Foundation programme (Y1MPharmW/Foundation) students attended their first autumn term meeting.

This study evaluated students' perceptions of the support offered, with the aim to understand how to make the ATS more engaging. The ultimate aim is to ensure effective support during transition to university, promote students' commitment with their study, improve academic attainment and retention.

Method/Summary of work: A meeting with Y1MPharmW/Foundation was organised where students were initially reminded of the ATS's principle including students' and staffs' expectations and responsibilities. An interactive presentation and a real-time voting questionnaire (mentimeter.com software) was used to (1) understand the reasons for the poor attendance; (2) gather feedback on the current practice; and (3) obtain suggestions on possible interventions that would promote students' participation. Answers to questions were quantified and expressed as \% of the total responses or analysed using thematic analysis. 
Of the students that completed the survey, 53\% (30/57) attended the meeting and $57 \%$ found it useful. A total of $42 \%$ students claimed that did not attend the tutor meeting as they did not perceive it as valuable or had no matters to discuss; of these, $83 \%$ claimed they would contact their tutor if necessary. $44 \%$ of the students confirmed that they would attend the meeting if scheduled within their T\&L activities, but $30 \%$ of students would consider attending only if there were not clashes with personal commitments. Comments highlighted the need for setting topic-specific conversations and activities, and even prizes for attendance.

Results/Discussion: Students comments and proposed ideas, suggested that students failed to recognise the value of the ATS. The academic tutor meeting is often perceived as a resort to turn to only if concerns raise. Having centrally timetabled and more structured meetings may promote engagement with the system.

Conclusion(s): A clearer explanation of the ATS's purposes, the official arrangements of meetings, a defined agenda, specific points for discussion and activities, will help students to fully appreciate and benefit from the support offered by the ATS.

\section{REFERENCE(S)}

1. Grant, A. 2006. "Personal tutoring: a system in crisis." In Personal Tutoring in Higher Education, edited by L. Thomas and P. Hixenbaugh, 11-20. Stoke on Trent, UK: Trentham Books

2. McFarlane, K. J. 2016. "Tutoring the tutors: supporting effective personal tutoring." Active Learning in Higher Education 17 (1): 77-88. oi: 10.1177/1469787415616720.

3. Thomas, L. 2012. Building Student Engagement and Belonging in Higher Education at a Time of Change. Paul Hamlyn Foundation. Vol. 100. London: Paul Hamlyn Foundation.

4. McCary, J., D. B. Johnstone, H. Valentine, and A. Berry. 2011. "A Comparative Evaluation of the Roles of Student Advisor and Personal Tutor in Relation to Undergraduate Student Retention." Final Report. Cambridge, UK: Anglia Ruskin University)

\section{P268 | PharmacoloGenius: Development of a mobile application to engage in pharmacology}

Claudia Pisanu' ${ }^{1}$; Gianpaolo Zammarchi ${ }^{2}$

${ }^{1}$ Department of Biomedical Sciences, University of Cagliari; ${ }^{2}$ Department of Economics and Business Science, University of Cagliari

Introduction/Background \& Aims: Mobile applications represent novel instruments to spread education and to communicate. One of the most appreciated features of these applications is that they only require a few minutes per day, as they are able to engage the user in the long-term. Some of these applications implement games, which often represent a smart solution to increase the motivation of the users and let them actively learn and practice. Although mobile games are increasingly being used as learning tools, this approach has not been applied to engage in pharmacology yet. This project, supported by a grant from the British Pharmacological Society (BPS), aims to develop
PharmacoloGenius, a mobile application implementing different games to let students increase their knowledge about pharmacology.

Method/Summary of work: The application will be developed in the Java language, using the multi-language and multi-platform Android Studio integrated development environment (IDE). To ensure the highest compatibility with different Android devices, testing and debugging will be conducted using both real Android smartphones and tablets, as well as different emulated Android Virtual Devices. Further testing will be conducted after publication of the application on Google Play Store and collection of users' suggestions. Contents (e.g., questions and explanations) will be developed in accordance with the BPS core curriculum, using the following sources as references: The Concise Guide to Pharmacology 2017/2018, the IUPHAR/BPS Guide to Pharmacology database, and the Goodman and Gilman's The Pharmacological Basis of Therapeutics 13th textbook.

Results/Discussion: Different games are currently being developed, e.g., quiz based on the topics of the BPS Undergraduate core knowledge curriculum (in case the user answers in a wrong way, the right answer and a short explanation will be provided) or memory games (consisting in combining two images showing related items, such as the name of a drug and its class). The games will test knowledge and understanding of theoretical principles of drug action, methodological principles, drug discovery \& development, the impact of pharmacology on society as well as related disciplines. The user will have the chance to gain points for each correct answer that will be provided.

Conclusion(s): PharmacoloGenius aims to be a useful instrument for undergraduate as well as graduate students that want to increase their knowledge in pharmacology in a fun and engaging way.

\section{P269 | The British Pharmacological Society undergraduate curriculum in a New Zealand setting}

Rachel Cameron; Malcolm Tingle

The University of Auckland

Introduction/Background \& Aims: The University of Auckland in New Zealand has restructured the Bachelor of Science degree to introduce (from 2021) a requirement for all students to complete a capstone course in their chosen major. A capstone is a student-led culminating course, which demonstrates achievement of majorspecific knowledge, skills, and attitudes, and helps students integrate concepts across the curriculum into a suite of skills and knowledge they can articulate to an employer and use to solve real world problems. To ensure a student-led experience, capstone courses are not permitted to include new disciplinary knowledge or didactic teaching methods.

This project aims to demonstrate the use of the British Pharmacological Society's undergraduate curriculum to inform the design of 
learning outcomes, learning activities and assessments, appropriate for teaching concepts and theories in pharmacology in the context of New Zealand populations.

Method/Summary of work: Since successful completion of a capstone requires students to integrate and apply previously learned knowledge and skills, using Brunner's spiral curriculum as a framework, we have chosen to redesign the entire suite of undergraduate prerequisite pharmacology courses to ensure adequate preparation. Three new courses are planned focused on: (1) molecular pharmacology, (2) pharmacokinetics and drug safety, and (3) preclinical and clinical trial evidence to support drug discovery and development. Key disciplinary knowledge, attitudes and skills expected from pharmacology graduates, were identified using the British Pharmacological Society undergraduate curriculum and used to guide the course design process.

Results/Discussion: This is a long-term project with step-wise implementation over three years. As a first step, a pilot pharmacology capstone course has been created for delivery in 2020 to a small number of students.

Conclusion(s): This project provides an opportunity to enhance international teaching of pharmacology principles through adaptation of a British curriculum to the unique social context of New Zealand, with Treaty of Waitangi responsibilities to Māori as tanagata whenua.

\section{REFERENCE(S)}

1. Bruner, J. S. (1960). The Process of Education. Cambridge, Mass.: Harvard University Press.

\section{P270 | Evaluating assessment strategies in Pharmacology teaching}

\section{Derek Costello; Carmel Hensey}

University College Dublin

Introduction/Background \& Aims: The current study was undertaken in response to concerns of overassessment within undergraduate teaching in Pharmacology. We sought to investigate the extent of assessment carried out during year 3 of study, and to acquire an overview of the assessment portfolio employed within the core components of the Pharmacology curriculum. Moreover, we endeavoured to interrogate the effectiveness of extensively-used assessments, with regard to overall student learning and alignment with the programme outcomes.

Method/Summary of work: We undertook an analysis of both the number and type of assessments completed by students in Stage 3 of our Pharmacology programme. Analysis was restricted to 7 taught modules with core Pharmacology-based content, which must be completed to comply with the requirements of the programme. Information was requested from individual module co-ordinators through population of a spreadsheet table. In the initial instance, general information relating to module assessment was obtained, including numbers and methods of assessment, and respective grade weighting (academic year 2017-2018). Based on initial analysis, a second request was issued to acquire information relating to overall module grades and average grades for individual assessment components. To determine alignment of assessment strategies to programme outcomes, the "UCD Pharmacology Programme Outcomes" were evaluated against the skills measured via both practical reports and project-based tasks. Three academic contributors to the Stage 3 Pharmacology curriculum considered the skills addressed by each assessment strategy, and to what extent these address each programme outcome.

Results/Discussion: Our analysis identified a varied and comprehensive assessment strategy within core Pharmacology teaching; consisting of practical reports, laboratory tests, multiple choice questions, essay-style exams and project-based tasks. However, we further revealed a substantial assessment burden imposed upon students, which is largely based on the completion of written practical reports. Although the skills evaluated by these tasks address many of the Pharmacology programme outcomes, no apparent benefit is obtained from either their quantity or repetition. Moreover, practical reports compare equivalently to lesser-used, active-learning methodologies for addressing programme learning goals.

Conclusion(s): We propose that a revised assessment strategy which reduces the number of practical reports in favour of formative components, and one which encourages student engagement with feedback, will likely support quality of learning and student satisfaction. We are currently investigating the introduction of novel strategies to assess practical work in the context of project-based learning; suitable for large-scale teaching.

\section{P271 | Pupil-led biomedical lessons for primary school children-A pilot study}

\author{
Fiona Murray ${ }^{1}$; Claire Aitken ${ }^{2}$; Derek Scott ${ }^{1}$ \\ ${ }^{1}$ University of Aberdeen; ${ }^{2}$ Broomhill Primary School, Aberdeen
}

Introduction/Background \& Aims: In 2017, the Scottish Government published a strategy that recommended increasing science education at primary school level ${ }^{[1]}$. Our institution had previously developed a large range of activities to help older pupils learn about pharmacology and physiology, but we had little experience with younger students. This pilot study aimed to develop a series of activities for primary school pupils and teachers, with children encouraged to take the lead in presenting what they had learned.

Method/Summary of work: Primary 2 children $(n=25)$ were given the opportunity to trade in classroom points for a lesson on a variety of biomedical topics. Pupils could choose to present a topic as a 2-3 slide PowerPoint presentation/poster and help organize 1-2 activities for the class. Resources and possible activities were provided by the University of Aberdeen but pupils had to design the activity themselves. Topics included the brain and heart. For the brain, pupils presented posters, made neurons from pipe cleaners, built brain hats 
(shows different regions of the brain and associated functions) and discussed what maintains brain health. For the heart, pupils described heart functions, used red and blue wool to show the network of arteries and veins in the body, found their pulse, and discussed cardiovascular health and medicines.

Results/Discussion: The success of the first event led to more being requested by the school. Quotes from teachers included "all of room 9 were very, very engaged during the pupil led lesson about the brain," "the student told the class about the brain and organised children into groups to complete activities," "very successful lesson," "another fantastic pupil-led lesson about the heart, enjoyed by the whole class," "after one student led the lesson others wanted to do similar activities." Due to the success of this event, we plan to offer such classes to other primary schools and are putting together science activity packs that we can send to schools across the north of Scotland. These would include instructional videos to help primary teachers in remote and rural areas undertake such biomedical activities even when university staff cannot take part.

Conclusion(s): This pilot study demonstrated that involving primary school students in biomedical learning activities can foster leadership, improve presentation skills, encourage pupil-led research, and stimulate interest in pharmacology and physiology at a very young age.

\section{REFERENCE(S)}

1. Scottish Government (2017) Science, Technology, Engineering \& Mathematics Education and Training Strategy for Scotland https://www2. gov.scot/Resource/0052/00526536.pdf

\section{P272 | Development of a public engagement activity to raise awareness of pharmacology- A partnership with a botanic garden}

\section{Derek Scott; Rebecca Breen}

University of Aberdeen

Introduction/Background \& Aims: There is increasing demand for universities to undertake public engagement work so that the population learns more about research. One of the problems our pharmacologists face is that much of their research takes place in secure buildings and it can be difficult to hold events that are accessible to large groups/families. The University of Aberdeen Botanic Gardens were keen to raise their local profile and have the ultimate ambition of creating a physic garden to educate the public about the medicinal properties of plants. This project aimed to develop a public engagement activity within the botanic gardens that would attract visitors and educate them about the pharmacological properties of plants.

Method/Summary of work: Twelve infographic boards were designed to provide information about specific plants within the gardens that either had proven clinical/experimental benefits, or disputed efficacy. These were designed to be understood by visitors with no science education and included QR code weblinks to research articles if guests wished to learn more. The infographic boards were designed so their content could be easily changed and moved around the gardens to take advantage of whatever was in bloom during different times of year. Maps of the "Pharmacology Trail" with activities and questions were produced so that visitors and school parties could test their knowledge of pharmacology. The standard botanic garden visitor feedback questionnaires were adapted for this activity, and visitors anonymously deposited their responses in a locked box within the gardens. The responses were then analysed.

Results/Discussion: During the first two weeks of the Pharmacology Trail, 68 feedback forms were received. $40 \%$ indicated they had come just to see the trail. Twenty-five of the forms provided suggestions of plants that could be featured in future trails, with 43 different medicinal plants mentioned. 42 responses made comments that indicated they had not realized that plants could be a source of "proper medicines." $85 \%$ of responses said the trail had increased their knowledge of pharmacology and $100 \%$ wanted more of these trails around the gardens. Garden staff have reported an increase in the number of overall visitors, many asking specifically for the trail, and an increase in social media traffic.

Conclusion(s): This project has shown that pharmacologists can work in partnership with botanic gardens to enhance public understanding of pharmacology. It has also enhanced the diversity of the local population we can engage with and provided pilot data we can use to design future pharmacology educational events.

\section{P273 | Student-led gamification of pharmacology revision is perceived positively by peers}

Derek Scott; Cameron Malcolm; Jack Kirkman; Fiona Murray; James Hislop

University of Aberdeen

Introduction/Background \& Aims: Gamification is a teaching methodology where learners may be motivated to engage more effectively by applying gaming mechanics to traditional educational activities. As well as helping students learn more effectively, we hoped such a gamification approach would improve student interactions as some of our classes have highly diverse student groups. We had identified that some students had issues with some elements of remembering drug names, mechanisms of action and uses, particularly if there had been an extended period between their last lectures on such topics. Upon exploring some of the easiest options available to introduce gamification, we decided to use the Quizlet application, given that it incurred no major financial outlay, and seemed to be relatively easy to use by staff and students alike.

This pilot study aimed to investigate whether use of Quizlet could improve student engagement with and perception of the Drug Mechanisms element of the Pharmacology Objective Structured Practical Examination (OSPE) in our Honours class. 
Method/Summary of work: Summer research students used the Quizlet app to develop a large question bank to help their peers revise. Content was checked by academic staff. Use of Quizlet was piloted with a small class ( $n=22,20$ participated). Student performance could be private or they could compete on a leaderboard with classmates if they wished. Student perceptions were gathered after the assessment's grades and feedback had been reported to them. Anonymised data were analysed from Likert scores and free text comments.

Results/Discussion: $50 \%$ of the group reported using the app as much as they could, with only 1 participant saying they had not used it at all. $90 \%$ felt that gamification apps were useful revision aids and $85 \%$ reported that they felt staff should offer similar revision activities in other classes. All students chose to compete publicly on a leaderboard. Free text comments indicated that users seemed to appreciate the fact that they could keep preparing for the assessment in their own time, regardless of location, particularly if they had employment or caring commitments. The variety of different testing/gaming activities seemed to appeal to different users, who each had their own preferences regarding which game within Quizlet was most useful for their own learning methodology.

Conclusion(s): This pilot study has encouraged us to increase use of gamification applications such as Quizlet and explore further ways in which gamification could be introduced into our teaching and learning activities in partnership with students.

\section{P274 | Formative assessment as a tool to enhance PGT students' engagement with pharmacokinetics}

\section{Louise Miller}

University of Glasgow

Introduction/Background \& Aims: Developing knowledge and understanding of the pharmacokinetic principles (absorption, distribution, metabolism, and excretion) underlying drug-dose calculations is often perceived as a challenging and complicated topic. ${ }^{[1]}$ For students enrolling onto postgraduate taught (PGT) programmes, engagement with new academic content and associated assessment may also present as a key transitional barrier; particularly in subjects that do not directly align with previous qualifications.

Pedagogical practices designed to develop student assessment literacy have shown to enhance student learning outcomes and are considered best practice. ${ }^{[2-5]}$ Despite this, assessment and feedback is regularly the lowest scoring core area of Postgraduate Teaching Experience Survey (PTES). ${ }^{[6,7]}$

MED5129 Drug Disposition is a semester a one course delivered to PGT pharmacology students. Course content includes pharmacokinetic principles underlying Clinical Pharmacology and Therapeutic Drug Monitoring dose calculation. In 2018-2019, a series of formative pharmacokinetic assessment and coursework submissions were introduced to support student learning. This project aimed to investigate PGT student's perceptions of formative assessment and subsequent feedback received as a tool for enhancing preparation for summative assessment.

Method/Summary of work: Following delivery of MED5129 course content, all students (58) were invited to participate in an anonymous online questionnaire containing both 5-point Likert scale and openanswer questions. Nine participants provided consent and submitted questionnaire responses. Qualitative data was analysed thematically. Ethical approval for the project was granted by the University of Glasgow College of Medical, Veterinary and Life Sciences Ethics Committee.

Project findings indicate that course formative assessment was perceived as a positive tool for enhancing student transition to PGT study. Furthermore, formative assessment opportunities enhanced students' understanding of pharmacokinetic principles and calculations; $88.9 \%$ of responses strongly agreed/agreed that Pharmacokinetic formative assessment aided their understanding of the associated topic: "I gained deep understanding as well as confidence."

In addition, $67.7 \%$ respondents were initially unfamiliar with University/Programme-level assessment criteria and this was improved following engagement with formative feedback. However, there was no clear indication of preference for PK feedback delivery, with all provided perceived as beneficial.

Results/Discussion: Project outcomes influenced the re-design of course delivery. For 2019-2020, additional learning activities are planned to enhance PGT students' engagement with pharmacokinetic content and TDM calculations as well as formative feedback provided. This project provides preliminary data for further pedagogical investigation for enhancing PGT pharmacology students' academic engagement and assessment literacy.

Conclusion(s): Project outcomes contribute to both the scholarship of teaching and learning and to enhancement of pharmacology education. Whilst findings presented are transferable to a range of learning contexts, they are particularly relevant for delivery of Postgraduate Taught courses as transition into PGT study is enhanced by delivery of formative assessment feedback to develop students' assessment literacy and self-efficacy.

\section{REFERENCE(S)}

1. Brackett C.C., Reuning R.H. Teaching Pharmacokinetics using a student-centered, modified mastery-based approach. Am J Pharm Educ. 1999;63.

2. O'donovan B, Price M, Rust C. Know what I mean? Enhancing student understanding of assessment standards and criteria. Teach High Educ. 2004;9(3):325-335. https://doi.org/10.1080/1356251042000216642

3. Smith CD, Worsfold K, Davies L, Fisher R, McPhail R. Assessment literacy and student learning: the case for explicitly developing students 'assessment literacy.' Assess Eval High Educ. 2011;38(June 2015):1-17. https://doi.org/10.1080/02602938.2011.598636

4. Bovill C, Cook-Sather A, Felten P, Millard L, Moore-Cherry N. Addressing potential challenges in co-creating learning and teaching: overcoming resistance, navigating institutional norms and ensuring inclusivity in student-staff partnerships. High Educ. 2016;71(2):195208. https://doi.org/10.1007/s10734-015-9896-4 
5. Deeley SJ, Bovill C. Staff student partnership in assessment: enhancing assessment literacy through democratic practices. Assess Eval High Educ. 2017;42(3):463-477. https://doi.org/10.1080/02602938.2015. 1126551

6. Leman J. Trends in the taught postgraduate experience The Postgraduate Taught Experience Survey 2016. High Educ Acad. 2016.

7. Leman J. 2018 Postgraduate Taught Experience Survey. High Educ Acad. 2018. https://www.heacademy.ac. uk/system/files/downloads/Advance HE PTES 2018 report.pdf.

\section{P275 | Spoilt for/by (multiple) choice? Multiple answer: An alternative format for multiple choice questions}

\section{Richard Prince}

University of Manchester

Introduction/Background \& Aims: BIOL10822 Drugs: Molecules to Man is a level one pharmacology unit (enrolment $>500$ Biological Sciences students). It has traditionally been assessed using a multiple choice question (MCQ) exam, with questions requiring students to select either the one correct or one incorrect option from four choices. To avoid students benefiting by simply guessing, we adjust marks using negative marking: for each incorrect response $1 /(n-1)$ marks is deducted (where $\mathrm{n}$ is the number of options).

Negative marking is poorly received by students who see it as "stealing marks." It also forces students to guess in order to take advantage of partial knowledge, e.g., a student able to eliminate one option from four as a possible answer must guess between the remaining options and will, on average, score $11 \%$. However, many students (females in particular [1]) are reluctant to use this strategy and instead omit the question.

Method/Summary of work: I converted 11 one incorrect option (OIO) questions from the 2015 paper to a new format, termed the "Multiple Answer Question" (MAQ). In MAQs, students have four options, three of which are correct. For each correct option they select, they gain $1 / 3$ of a mark. If they select the one incorrect response, 1 mark is deducted. I deployed these questions in the 2019 unit examination alongside 20 standard "one correct" questions from 2015. Question formats were compared by score and \% students omitting question (Table).

NSD: no significant difference 2019 vs 2015 standard MCQ, Student's paired $t$ test. ${ }^{\dagger} P=0.001$ multiple answer vs one incorrect, Student's paired $t$ test; ${ }^{*} P=0.0362019$ vs 2015 standard MCQ, Wilcoxon matched pairs signed rank test, ${ }^{* *} P=0.001$ multiple answer vs one incorrect, Wilcoxon matched pairs signed rank test.
Results/Discussion: The MAQ format yields a higher average mark with fewer students omitting the question, compared to OIO questions. These differences may be due to the MAQ format rewarding partial knowledge more, without forcing students to guess. A student who can identify one correct option gains $33 \%$ of the marks in a MAQ (without guessing), compared to $11 \%$ (with guessing) for a question in the one incorrect format.

The MAQ approach is conceptually similar to "Elimination Testing" (ET). However, ET questions are less intuitive to answer because students gain marks by eliminating incorrect options. Similar to my results, ET questions result in higher scores and fewer question omissions [2].

Conclusion(s): The MAQ format offers an alternative to traditional MCQs that has the potential to improve students' marks by rewarding partial knowledge and removing the need to guess.

\section{REFERENCE(S)}

1. Ben-Shakhar, G. and Sinai, Y. (1991) J. Educ. Meas. 28. 23-35

2. Ben-Simon, A., Budescu, DV. And Nevo, B. (1996) Appl. Psychol. Meas. 21: $65-88$

\section{P276 | The importance of therapeutic compliance: An innovative teaching proposal}

Tatiana Ignatova ${ }^{1}$; Marta Elias ${ }^{2}$; Pau Solà ${ }^{2}$ Irene Forment ${ }^{2}$; Maria Ángeles Garcia²; Laura Cuffi ${ }^{2}$

${ }^{1}$ University of Barcelona CIF Q0818001J; ${ }^{2}$ University of Barcelona

Introduction/Background \& Aims: We present a novel educational proposal, carried out at the University of Barcelona, based on a service-learning perspective in medical education (Stewart \& Wubbena, 2014). Service-learning integrates the study of academic contents with university service to society; learning improves service to the community and the service gives meaning to learning. This Service-learning project aims to disseminate the importance of a responsible use of drugs; best medications do not ensure the expected result if there is a lack of therapeutic compliance. The Project considers the Sustainable Development Goals defined by UNESCO.

Method/Summary of work: First, Health Sciences students investigate the degree of therapeutic compliance in their social environment, identify a real need to act upon and devise an action plan with the available resources. Then, a collaborative work begins with Media students to elaborate an audio-visual product which includes the

\section{TABLE 1}

\begin{tabular}{|c|c|c|c|c|}
\hline & \multicolumn{2}{|l|}{2019 Examination } & \multicolumn{2}{|l|}{2015 Examination } \\
\hline & $\begin{array}{l}\text { Standard one correct } \\
\text { MCQ }\end{array}$ & $\begin{array}{l}\text { Multiple answer } \\
\text { question }\end{array}$ & $\begin{array}{l}\text { Standard one correct } \\
\text { MCQ }\end{array}$ & $\begin{array}{l}\text { One incorrect } \\
\text { question }\end{array}$ \\
\hline Mean $\%$ score per question $( \pm$ SEM) & $77 \pm 2$ NSD & $63 \pm 5^{\dagger}$ & $73 \pm 3$ & $53 \pm 6$ \\
\hline Median \% students omitting question (inter-quartile range) & $3.2(2.4-7.2)^{*}$ & $4.7(2.9-11.4)^{* *}$ & $3.9(2.4-13.6)$ & $12.9(7.6-20.9)$ \\
\hline
\end{tabular}


therapeutic objectives and will be distributed in hospital waiting rooms. Three audio-visual pieces related to antibiotic consumption and neuropathic ulcers and dermatomycosis drug treatments have been developed since the academic year 2016-2017. Generic skills about how to communicate scientific concepts in a language style appropriate to the subject and audience and to network with experts from another field of knowledge are achieved. Pharmacological acquired skills are to detect the factors causing a lack of adherence to treatment by the patient and to select and rank the most important information about the targeted pharmacological group (indications, therapeutic use and circumstances that prevent poor therapeutic compliance).

Results/Discussion: The educational and social success of this project encourages it to continue. However, working with students and teachers from two different faculties is not easy. The university system does not contemplate either the evaluation of these integrated interdisciplinary projects in the academic curriculum. A lot of time is required, and greater economic resources would be needed.

Conclusion(s): Teaching of pharmacology is enriched by the contextualization of health problems from a community perspective, strategies to promote responsible use of drugs, the ability to formulate, design and manage projects and to communicate pharmacological contents, autonomous learning and insight of health information systems. Service-learning involves an educational tool enabling an interdisciplinary project, which involves a joint work between teachers and students of different areas of knowledge, with a social impact.

\section{REFERENCE(S)}

1. Stewart, T. and Wubena, Z. (2014) "An overview of infusing servicelearning in medical education", International Journal of Medical Education, 5: 147-156.

\section{P277 | Using principles of Universal Design for Learning (UDL) to improve student engagement in a veterinary clinical pharmacology module}

\section{Deirdre Campion}

University College Dublin

Introduction/Background \& Aims: For the past two decades, research into educational inclusion has looked beyond students with disabilities and has sought to create an educational environment where all

\section{TABLE 1}

\begin{tabular}{|c|c|c|c|c|c|c|c|}
\hline Year & $\begin{array}{l}\text { Class } \\
\text { size }\end{array}$ & $\begin{array}{l}\text { Response } \\
\text { rate }\end{array}$ & $\begin{array}{l}\text { Q1. I have a better } \\
\text { understanding of the } \\
\text { subject after completing } \\
\text { this module }\end{array}$ & $\begin{array}{l}\text { Q2. The } \\
\text { assessment was } \\
\text { relevant to the } \\
\text { module }\end{array}$ & $\begin{array}{l}\text { Q3. I achieved } \\
\text { the learning } \\
\text { outcomes }\end{array}$ & $\begin{array}{l}\text { Q4. The teaching on } \\
\text { this module supported } \\
\text { my learning }\end{array}$ & $\begin{array}{l}\text { Q5. Overall I am } \\
\text { satisfied with } \\
\text { this module }\end{array}$ \\
\hline '17-18 & 110 & $18.9 \%$ & 3.45 & 3.3 & 3.1 & 3.15 & 3.05 \\
\hline '18-19 & 128 & $21.9 \%$ & 4.07 & 4.43 & 3.82 & 3.89 & 3.79 \\
\hline
\end{tabular}

learners are provided with an equal opportunity to learn. Universal Design, a concept which originated in architecture, challenges the educator to consider that the "one size fits all" curriculum itself is "disabled." The principles of Universal Design for Learning (UDL) are grounded in scientific understanding of brain development, of learning, and of the different media forms now available to the educator [1]. The goal of this study was to use UDL to improve student engagement with a veterinary clinical pharmacology module.

Method/Summary of work: The module of concern occurs mid-way through the veterinary medicine degree and is normally attended by 110-130 students. The student cohort includes a mix of Irish and EUentry students who are mid-way through their first university degree, alongside EU and international students who had graduated with a scientific degree before entering the veterinary course. As a result, there is a diversity of experience, gender and culture in the classroom. Commonly, $10 \%$ of the cohort are registered to receive extra academic accommodations.

Using the principles of UDL, a number of interventions to improve engagement were instigated for the academic year 2018-2019: (i) $25 \%$ reduction in lecture content with increased assessment of independent directed learning; (ii) improved opportunities for self-assessment using clinically relevant MCQs (iii) clinicallyorientated assignments of staggered complexity, with the second and third assignments exploiting skills that had been developed in the previous assignments; (iv) development of an "aide-memoire" for use in class and in the final examination to eliminate the need for memorisation of drug names and groups.

Student satisfaction with the module was evaluated using an institutional anonymous, standardised electronic survey based on a 5 -point Likert scale where 1 = strongly disagree, and 5 = strongly agree.

Results/Discussion: Following these interventions, student satisfaction with the module was greatly increased. In addition, individualised feedback led to a stepwise improvement of student grades with each assignment.

Conclusion(s): Student satisfaction with this veterinary clinical pharmacology module was improved following the adoption of UDL principles. Also, by rewarding student effort through feedback, student outcomes were improved. UDL principles are applicable across all disciplines but are especially relevant to content-heavy courses such as clinical pharmacology.

\section{REFERENCE(S)}

1. Meyer, A., Rose, D.H., \& Gordon, D. (2014). Universal design for learning: theory and Practice. Wakefield, MA: CAST Professional Publishing. 


\section{P278 | Attitudes of medical students towards pharmaceutical industry}

Kristian Dominik Rudez ${ }^{1}$; Igor Radanovic ${ }^{1}$; Deni Rkman ${ }^{1}$; Jeroen van Smeden ${ }^{2}$; Robert Likic ${ }^{3}$

${ }^{1}$ School of Medicine, University of Zagreb; ${ }^{2}$ Centre for Human Drug Research, Leiden; ${ }^{3}$ University Hospital Centre Zagreb, Department of Internal Medicine, Division of Clinical Pharmacology and Therapeutics; School of Medicine, University of Zagreb

Introduction/Background \& Aims: Interaction between physicians and the pharmaceutical industry has been present for decades and can lead to biased prescribing habits and lowered patient trust. It has also been shown that the first interactions with industry representatives start at medical school, with considerable inter institutional variation in the amount of education on this topic. The aim of this study was to explore the final year medical students' knowledge and attitudes towards interactions with the pharmaceutical industry, before they start working independently.

Method/Summary of work: An online-based survey was conducted in June 2019 among all final year medical students at the University of Zagreb School of Medicine. The survey consisted of Likert scale-based questions aimed at assessing attitudes and motivations of students towards the pharmaceutical industry.

Results/Discussion: The overall response rate was $73.1 \%$ (226 out of 309 final year medical students). More than a third of the students (37.2\%) talked to a pharmaceutical representative in the past 6 months, while $53.1 \%$ observed an attending physician or resident interacting with a pharmaceutical representative. Also, $39.4 \%$ of the students confirmed they had received a pharmaceutical industry related gifts. While $35.4 \%$ of students believed that their colleagues who received food/gifts from pharmaceutical representatives had an increased chance of eventually prescribing in favor of the company's product, significantly fewer (19.5\%) thought the same about themselves. About half of the surveyed students (56.2\%) considered drug company materials as a useful way to learn about new drugs, although the majority (58.8\%) believed that drug company-sponsored meetings were often biased in favor of the company's product. Most of the students (78.2\%) are not aware of their medical school having a policy regulating trainee-industry interactions. Only $10.2 \%$ stated they were educated about professional relationships with the industry. Opinions are divided on whether medical schools should permit pharmaceutical industry access to their students: $48.7 \%$ of the students think they should have access, while the rest of the students oppose this idea. Conclusion(s): The questionnaire indicated large differences in attitudes among medical students. They have not been systematically educated about interactions with the industry, despite the fact that the pharmaceutical industry may soon be involved in their careers and influence their daily prescribing. To be fully prepared for their starting career, education on proper communication with pharmaceutical representatives should be introduced in all medical schools' curricula.
Poster Session: Toxicology

\section{P279 | Population pharmacokinetics of gentamicin in puppies diagnosed with parvovirus enteritis}

Fidelis Gberindyer ${ }^{1}$; Matthew Abatan ${ }^{2}$; Felix Shima ${ }^{3}$; Paolo Denti ${ }^{4}$

${ }^{1}$ Federal Uinversity of Agriculture; ${ }^{2}$ University of Ibadan; ${ }^{3}$ Gan-Rovet Animal Hospital, Warri; ${ }^{4}$ University of Cape Town

Introduction/Background \& Aims: Gentamicin is a concentrationdependent antibiotic indicated with other drugs in managing canine parvovirus enteritis (CPE) [1, 2]. This study evaluated effect of comedications and some covariates of puppies with CPE on pharmacokinetics of gentamicin.

Method/Summary of work: Fifty puppies of different breeds with CPE were administered a single intramuscular dose of gentamicin at $6.0 \mathrm{mg} / \mathrm{kg}$. Sparse blood samples were obtained over 24 hours and gentamicin concentrations quantified using ELISA. Population pharmacokinetics was evaluated employing non-linear mixed-effect modeling in MONOLIX ${ }^{\circledR}$ 4.3.3 to elucidate significant effect (objection function value > 3.84) of covariates on pharmacokinetics of gentamicin. Simulation of serum concentration-time profiles was performed with MLXPlore 1.1.1 using the developed model.

Results/Discussion: A two-compartment model with first-order absorption and elimination described pharmacokinetics of gentamicin (Figure 1). Age, clinical serum biochemistry, and co-medications covariates had no significant impact on the pharmacokinetics of gentamicin. However, allometric scaling with BWT and estimated exponents accounted well for effect of body size on clearance and volume of distribution (V). Also, large between-subject variability of $86 \%$ was observed in V. Simulations with dose levels $4-10 \mathrm{mg} / \mathrm{kg}$ showed trough serum concentrations within $0.46-1.47 \mu \mathrm{g} / \mathrm{ml}$ with corresponding steady state concentrations of $5.26-13.17 \mu \mathrm{g} / \mathrm{ml}$ at 2.6 hours post second dose.

Conclusion(s): Body weight was the only covariate affecting pharmacokinetics of gentamicin in our cohort of puppies. Our model indicates safe and effective serum concentrations at dose levels 6-9 $\mathrm{mg} / \mathrm{kg}$.

\section{REFERENCE(S)}

1. Prittie J (2004). Canine parvovirus enteritis: a review of diagnosis, management, and prevention. J Vet Emerg Crit Care 14(3): 167-176.

2. Kahn CM (2010). The Merck Veterinary Manual. 10th edn. 2945pp. Merck and Co. Inc., New Jersey. 
Oral Communications, Tuesday 17th December, 14:15

\author{
Education and Skills Oral Communications 1
}

\section{OC070 | Enhancement of the teaching of undergraduate pharmacology courses via development of computer based simulation programs}

\section{Abdalla Wedn; Sahar El-Gowilly \\ Faculty of pharmacy, Alexandria University, Egypt}

Background and Aims: Existing pharmacology simulation programs can be purchased and used for educational purposes. However, elearning software can be used by institutions to design programs tailored to educational needs of their students. In this study, we designed simulation programs to address specific students' misunderstandings identified during practical pharmacology sessions of previous years. We examined the impact of developing these programs on pharmacology knowledge of pharmacy students.

Summary of work and outcomes: Computer programs were developed and introduced for the cohort of undergraduate pharmacy students (approximately 900 students during academic year 2018/2019). Articulate Storyline 3 and iSpring Suite 8.7 were used to design practical experiments in the form of computerized simulation programs. These programs were supplemented with pre-made videos showing steps of in vivo or in vitro experiments. Additionally, Microsoft Excel sheets were utilized to construct dose response curves where applicable. The students' satisfaction with various simulation activities was assessed by means of anonymous surveys and their knowledge was evaluated by electronic quizzes. Students' results were compared with those of previous cohort (academic year 2017/2018) in which traditional methods were employed in practical sessions.

The majority of students were satisfied with transformation of wet lab sessions into computer based activities (89, 83, and $70 \%$ for virtual experiments, Excel sheets and videos, respectively). The percentage of students expressing their dissatisfaction ranged from $10 \%$ to $30 \%$ with the lack of familiarity with these new programs being the most reported reason. The students' performance and results in two main subtopics (GIT and vascular pharmacology) were significantly higher when compared with results from previous year.

Discussion: Implementation of simulation programs (i) enhanced students' understanding of basic pharmacology concepts as evidenced by elevation of students' grades and satisfaction and (ii) resulted in a significant cost saving for the department ( $>100$ LE per student) as a consequence of reduced wet lab burden. To optimize students' comprehension and experience, a combination of wet lab demonstrations and simulation activities will be used in the upcoming years in our department.

Conclusion: We recommend exploitation of simple electronic software to design comprehensive pharmacology-related simulation programs. These programs help to save time and cost associated with practical experiments as well as to enhance students' performance and understanding of the subject.

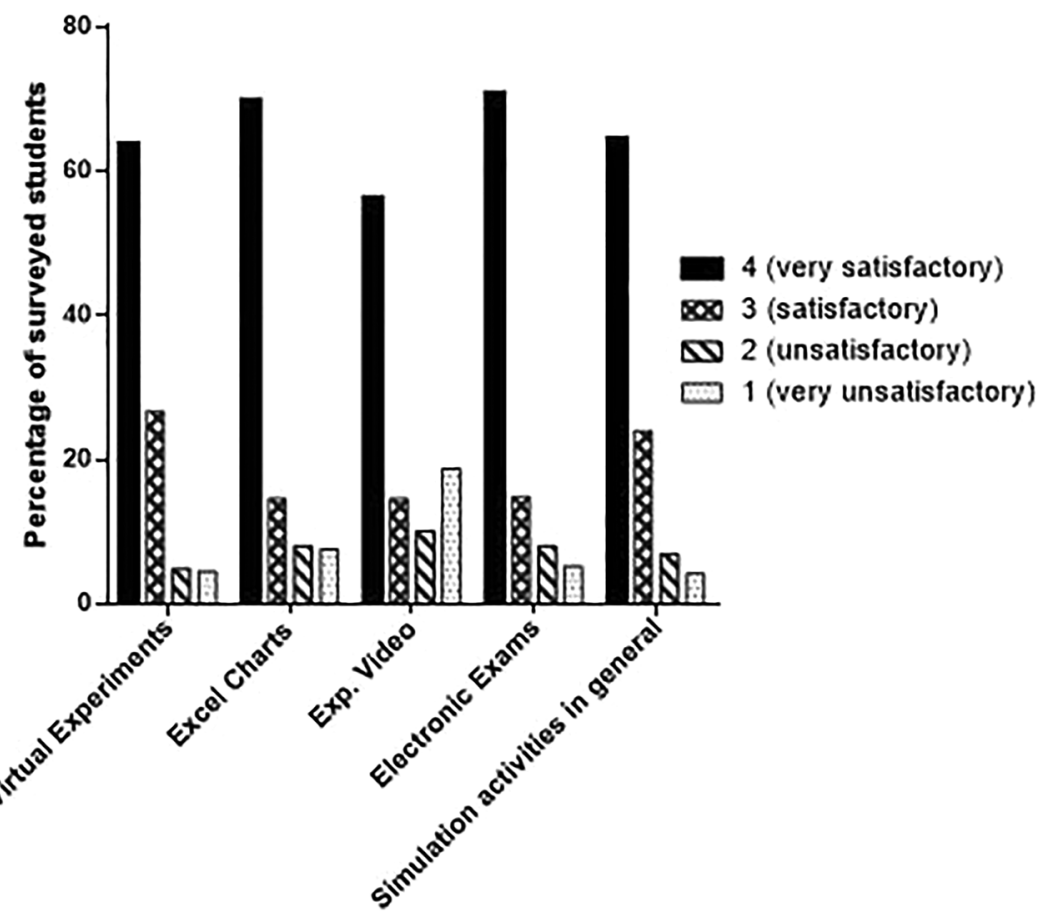

FIGURE 1. Student satisfaction with computer based learning activities 

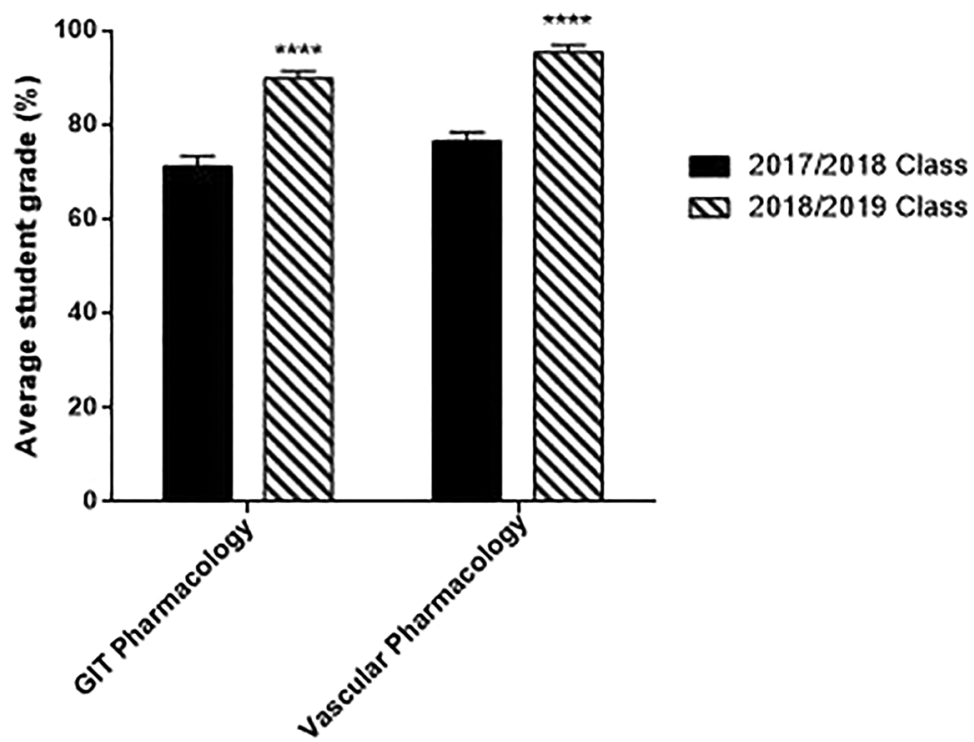

FIGURE 2. Comparison of student grades between two classes: $2017 / 2018$ (in which traditional teaching methods were employed) and $2018 / 2019$ (in which simulation activities were implemented). Values are means \pm SEM of 907 student grades, ${ }^{* * * *} p<0.0001$ vs. class of $2017 / 2018$

\section{OC071 | Revolutionising what pharmacology graduates need to know: Core concepts in pharmacology education}

Paul White ${ }^{1}$; Thomas Angelo²; Jacqueline McLaughlin²; Michael Munday ${ }^{3}$

${ }^{1}$ Monash University; ${ }^{2}$ University of North Carolina; ${ }^{3}$ University College London

Introduction/Background \& Aims: Over the past decade, the Faculty of Pharmacy and Pharmaceutical Sciences at Monash University, Australia, has transformed the education of pharmacists and pharmaceutical scientists by implementing program-wide, flipped classroom active learning [1], using concept mapping and scenario-based learning to develop critical thinking and communication skills in pharmacology [2], and validating or modifying these approaches. One significant gap remains: an evidence-based approach to deciding what knowledge we teach and assess. The development of concept inventories and threshold concepts in other disciplines [3] provides a clue to closing this gap.

Method/Summary of work: A staged approach has been designed at Monash, University College London, and the University of North Carolina to develop core and threshold concepts in pharmacology and related disciplines. First, experts have begun to identify a "draft set" of core pharmacology concepts (CPCs). So far, key exemplar concepts (identified by 15 experts at a conference in Italy) include drug-receptor interactions, antimicrobial resistance, and drug absorption. Going forward, we will "unpack" the CPCs to determine which underlying core science concepts (CSCs) from biochemistry, organic chemistry, physiology, etc. are prerequisite and necessary to CPC mastery. Third, we will develop valid and reliable concept inventories (MCQ tests) for assessment of learners' understanding of CSCs and CPCs.

Results/Discussion: The core concepts and MCQ banks will for the first time allow educators across the globe to develop new curricula using an evidence-based knowledge framework that provides clear direction for learning outcomes, teaching activities and high-quality assessments, and to test attainment using a bank of validated test items that permits apples-apples comparisons of teaching innovations. Conclusion(s): This project will offer pharmacology education a new evidence-based, concept-focused paradigm as an alternative to the traditional fact-focused approach.

\section{REFERENCE(S)}

1. White PJ, Larson I, Styles K, Yuriev E, Evans DR, Rangachari PK Short JL, Exintaris B, Malone, DT, Davie B, and Eise N (2016) Adopting an active learning approach to teaching in a research-intensive higher education context transformed staff teaching attitudes and behaviours. Higher Education Research \& Development 35619-633.

2. White PJ, Naidu S, Yuriev E, Short JL, McLaughlin JE, \& Larson IC (2017) Student engagement with a flipped classroom teaching design affects pharmacology examination performance in a manner dependent on question type. Am J Pharmaceutical Education, 81: 5931.

3. Hestenes, DMW, Swackhamer, G (1992) "Force concept inventory." The Physics Teacher 30: 141-158.

\section{Oc072 | The benefits of testing for enhancing student learning}

\section{N. Freestone; Ribin Kohnahpoushi}

Kingston University 
Introduction/Background \& Aims: It has been reported that using tests for student assessment not only evaluates students' current state of knowledge but also actively improves learning (Pyc and Rawson, 2010). Mangen et al (2013) have additionally suggested that student learning from any media source is dependent on the nature of the physical interaction between student and media source. We have previously reported a study that looked at higher education (HE) students at levels 4 and 6 (Years 1 and 3 ) to determine if there were differences manifested in their ability to accurately recall information from paper and digitally presented content as they progressed through their degree programmes. In this study, first year students performed better using paper-based sources of information, whilst for third year students, there were no differences manifested. The effect of media sources on longer-term student learning has not been evaluated however.

Method/Summary of work: Students undertook a comprehension test immediately after studying a text either using a mobile phone, iPad (or similar), or a laptop. This was followed at a later time point by (a) a test on thematically related material and (b) a test on thematically unrelated material.

Results/Discussion: Screen size was found to be an important factor for student learning, with mobile phones being the least beneficial (21.1\%; $\mathrm{n}=47$ versus 33.3\%; $\mathrm{n}=23 ; p=0.027$ for mobile phones and laptops, respectively). This relationship also held true for a later test involving thematically related material. However, in this situation, those students who did not undertake the first test performed on average significantly less well than their pre-tested peers $191.6 \%$; $\mathrm{n}=33$ versus $70.5 \% ; \mathrm{n}=25 ; p=0.038$ for tested versus non-tested students respectively). Even more noteworthy was the fact that marks on a test on thematically unrelated material yielded the same relationship although this did not reach statistical significance $(65 \% ; n=33$ versus $54.3 \%, n=25$ for tested versus non-tested students respectively). This data extends previous research, for example, McDaniel et al (2012) showed that exam marks were higher for psychology students exposed to practice questions compared to unpracticed questions for both repeated and non-related questions.

Conclusion(s): The results presented here, in a pharmacological context, indicate a robust way of improving educational outcomes more widely. Dunlosky et al (2013) evaluated different learning techniques in a variety of different learning contexts and found methods similar to that outlined above to be among the most effective in improving student learning.

\section{REFERENCE(S)}

1. Pyc MA and Rawson KA (2010) Why testing improves memory: mediator effectiveness hypothesis. Science, 335

2. Mangen A, Walgermo BR, Bronnick KK (2013) Reading linear texts on paper versus computer screen: effects on reading comprehension International Journal of Educational Research 58:61-68

3. McDaniel MA, Wildman KM, Anderson JL (2012) Using quizzes to enhance summative-assessment performance in a web-based class Journal of Applied Research in Memory and Cognition 1:18-26

4. Dunlosky J, Rawson KA, Marsh EJ, Nathan MJ, Willingham DT (2013) Improving students' learning with effective learning techniques: promising directions from cognitive and educational psychology. Psychological Science in the Public INterest 14:4-58

\section{OC074 | Final year undergraduate research project or a "Capstone Experience"? Time for a re-think?}

\section{Dave Lewis}

University of Leeds

Introduction/Background \& Aims: The QAA Biosciences Sciences Benchmark statements require the students to "have personal experience of hypothesis driven research such as a project/research based assignment" [1]. Typically, across the Sector, these have been laboratory or fieldwork projects or (critical) reviews of the literature. However, only a third of Bioscience graduates go onto careers in scientific or medical research and therefore there is a need for educators to rethink their expectations for "research projects," to provide alternative, enquiry-based learning opportunities for students that enable them to develop, utilise, and showcase the key employability skills required for careers outside of research. The aim of this work create and implement a suite of alternatives to traditional research projects into Biomedical Science degree programmes at the University of Leeds and evaluate the outcomes.

Method/Summary of work: Informed by our previous surveys of student and staff expectations for final-year research projects [2], a suite of nine non-traditional enquiry-based capstone experiences were introduced into undergraduate pharmacology, physiology, medical sciences and neuroscience degree programmes as additional alternatives to the existing five formats of traditional research projects. Feedback was sought from both students and graduates, and academic outcomes also determined.

Results/Discussion: Capstone alternatives to traditional research projects are extremely popular with students, with demand far exceeding supply (18 vs $8 \%$ ). There was no difference in student attainment; the mean module mark for both groups was $70 \%$. Reflecting on their project experiences and opportunities 1 year post graduation, three times as many graduates wished they had undertaken a non-traditional capstone experience that were able to ( $31 \%$ vs $10 \%$ ).

Capstone experiences enabled students to develop, apply and, most importantly, showcase the majority of employability skills required by graduate employers. There is a need to embed more opportunities to develop teamwork, leadership and commercial/entrepreneurial skills.

By providing students with the opportunity to undertake alternatives to traditional research projects, it enables them to decide what they want to achieve, personally and professionally, through their research project or capstone experience, and choose accordingly.

Conclusion(s): This study demonstrates the need for Bioscience educators to broaden their thinking and expectations for final-year 
undergraduate research projects; to embrace the concept of enquirybased capstone experiences rather than the narrower "research project," in line with the upcoming revised Royal Society of Biology accreditation criteria. In doing so, capstone experiences have the potential to be both transformational and translational.

\section{REFERENCE(S)}

1. Quality Assurance Agency qaa.ac.uk/en/Publications/Documents/SBS-Biosciences-15.pdf

2. Lewis DI et al. (2017). Proc Brit Phamacol Soc, Pharmacology P17 meeting $135 \mathrm{P}$ 